\title{
Rhodium(III) Catalyzed Carboamination of Alkenes Triggered by C-H Activation of $N$-Phenoxyacetamides Under Redox-Neutral Conditions
}

$$
\text { Zhiyong } \mathrm{Hu},{ }^{\dagger, *} \text { Xiaofeng Tong, }{ }^{*} \text { Guixia Liu, }{ }^{*}{ }^{\dagger}
$$

('State Key Laboratory of Organometallic Chemistry, Shanghai Institute of Organic Chemistry, Chinese Academy of Sciences, Shanghai 200032, China)

( Key Laboratory for Advanced Materials and Institute of Fine Chemicals, East China University of Science and Technology, Shanghai 200237, China)

E-mail: guixia@sioc.ac.cn

\section{Supporting Information}

\section{Contents}

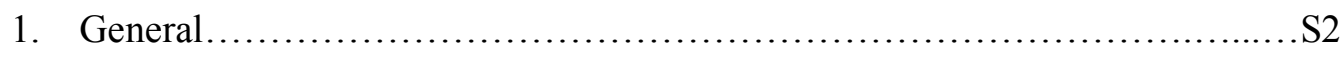

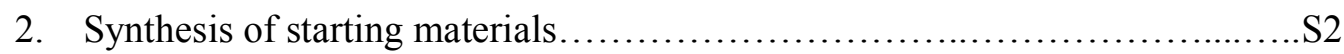

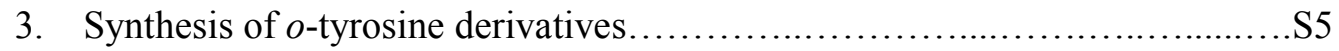

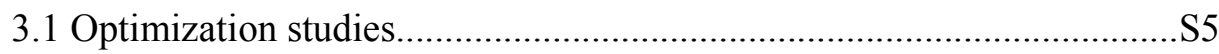

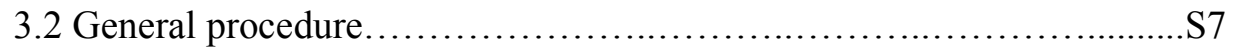

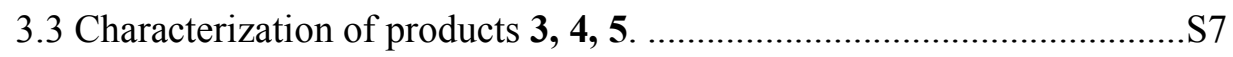

4. Deuterium-labeling experiments...................................... 14

5. References....................................................... 16

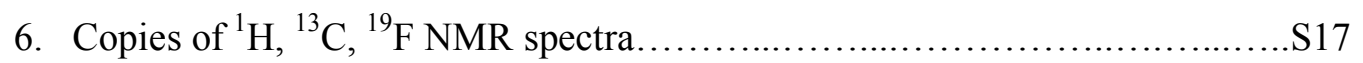




\section{General}

NMR spectra were recorded on a Varian Mercury Vx400 spectrometer in solvents as indicated. Chemical shifts $(\delta)$ are given in ppm relative to TMS. The residual solvent signals were used as references and the chemical shifts were converted to the TMS scale $\left(\mathrm{CDCl}_{3}: \delta_{\mathrm{H}}=7.26 \mathrm{ppm}, \delta_{\mathrm{C}}=77.16 \mathrm{ppm}\right.$; DMSO- $d_{6}: \delta_{\mathrm{H}}=2.50 \mathrm{ppm}, \delta_{\mathrm{C}}=39.52$ ppm, MeOD- $\left.d_{4}: \delta \mathrm{H}=3.31 \mathrm{ppm}, \delta \mathrm{C}=49.00 \mathrm{ppm}\right)$. Infrared spectra were obtained on a Bio-RadFTS-185 instrument. Mass spectra were provided on Agilent 5973 or Agilent 1100 instruments. All melting points were uncorrected. $\left[\mathrm{Cp} * \mathrm{RhCl}_{2}\right]_{2}$ was purchased from Strem. $4 \AA$ MS was activated in muffle furnace before use.

\section{Preparation of the starting materials}

$N$-aryloxyacetamides, ${ }^{[1,2]}$ alkenes $\left(\mathbf{2} \mathbf{h}^{[3]}, \mathbf{2} \mathbf{j}^{[4]}, \mathbf{2 l}^{[5]}, \mathbf{6}^{[6]}, \mathbf{7}^{[7]}, \mathbf{8}^{[8]}, \mathbf{9}^{[9]}, \mathbf{1 0}^{[10]}, \mathbf{1 1}^{[11]}\right)$ were synthesized according to the reported procedures and all data were in agreement with those reported.

\section{List of attempted alkenes:}
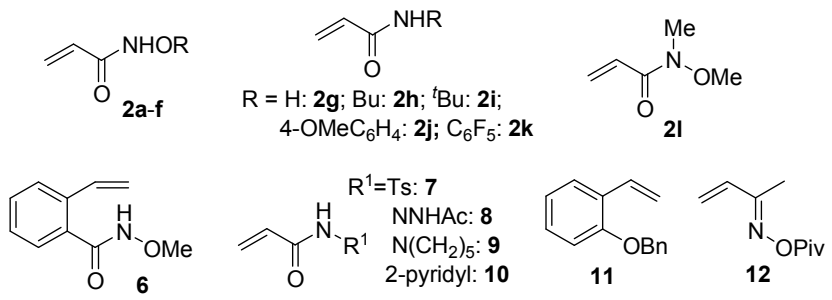

Among the attempted alkenes, only $\mathbf{2} \mathbf{a}-\mathbf{-}, \mathbf{2} \mathbf{j}$ and $\mathbf{2} \mathbf{k}$ could afford the desired $\mathrm{C}-\mathrm{C} / \mathrm{C}-\mathrm{N}$ formation products.

\section{Preparation of substrates $2 a$ and $2 b$}

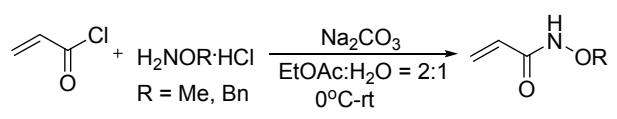

$O$-Alkylhydroxylamine hydrochloride ( 1 equiv) and $\mathrm{Na}_{2} \mathrm{CO}_{3}$ (2 equiv) were added to a mixture of EtOAc/ $\mathrm{H}_{2} \mathrm{O}(2: 1)$. The resulting mixture was cooled to $0{ }^{\circ} \mathrm{C}$, and acryloyl chloride (1 equiv) was added dropwise. Then the reaction was allowed to warm to room temperature. After 4 hours, the reaction was quenched with water, and the aqueous phase was extracted by EtOAc. The combined organic phase was dried over $\mathrm{Na}_{2} \mathrm{SO}_{4}$, filtered and evaporated under reduced pressure. The crude product was purified by flash column chromatography on silica gel to give the desired products as white solid.

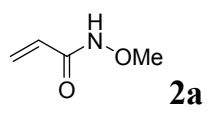

White solid, $90 \%$ yield $(1.82 \mathrm{~g})$ from $O$-methylhydroxylamine hydrochloride (20 mmol, 1.67 g, 1 equiv); m.p: $35-36{ }^{\circ} \mathrm{C} ;{ }^{1} \mathbf{H}$ NMR (400 MHz, $\mathbf{C D C l}_{3}$ ): $\delta 11.37$ (s, 1H), $6.39(\mathrm{~d}, J=16.8 \mathrm{~Hz}, 1 \mathrm{H}), 6.26(\mathrm{dd}, J=16.8 \mathrm{~Hz}, 10.4 \mathrm{~Hz}, 1 \mathrm{H}), 5.71(\mathrm{~d}, J=10.4 \mathrm{~Hz}$, 1H), 3.80 (s, 3H); ${ }^{13} \mathbf{C}$ NMR (100 MHz, $\left.\mathbf{C D C l}_{3}\right): \delta 163.8,127.3,127.2,63.6$; IR (neat, $\mathbf{~ c m}^{-1}$ ): $v$ 2968, 1663, 1507, 1064; MS (m/z, ESI): 102; HRMS calculated for $\mathrm{C}_{4} \mathrm{H}_{8} \mathrm{NO}_{2}(\mathrm{M}+\mathrm{H})^{+}:$102.055; Found: 102.0553; $\mathbf{R}_{\mathbf{f}}=0.1(\mathrm{EA}: \mathrm{DCM}=1: 4)$. 


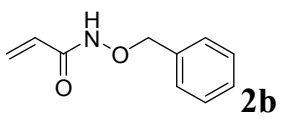

White solid, $86 \%$ yield $(1.52 \mathrm{~g})$ from $O$-benzylhydroxylamine hydrochloride $(10$ mmol, 1.59 g, 1 equiv); m.p: $56-57{ }^{\circ} \mathrm{C} ;{ }^{1} \mathbf{H}$ NMR (400 MHz, $\mathbf{C D C l}_{3}$ ): $\delta 10.37$ (s, 1H), 7.35-7.25 (m, 5H), $6.30(\mathrm{~d}, J=16.8 \mathrm{~Hz}, 1 \mathrm{H}), 6.15-6.09(\mathrm{~m}, 1 \mathrm{H}), 5.57(\mathrm{~d}, J=8.8 \mathrm{~Hz}$, 1H), 4.87 (s, 2H); ${ }^{13} \mathbf{C}$ NMR (100 MHz, $\left.\mathbf{C D C l}_{3}\right): \delta 163.7,135.2,129.1,128.6,128.5$, 127.4, 78.2 (one signal missing due to overlap); IR (neat, $\mathbf{c m}^{-1}$ ): $v 2981,1659,1629$, 1470, 1454, 1043, 746; MS (m/z, ESI): 178, 132, 121; HRMS calculated for $\mathrm{C}_{10} \mathrm{H}_{12} \mathrm{NO}_{2}(\mathrm{M}+\mathrm{H})^{+}:$178.0863; Found: $178.0864 ; \mathbf{R}_{\mathbf{f}}=0.6(\mathrm{EA}: \mathrm{DCM}=1: 4)$.

Preparation of substrates $2 \mathrm{c}-2 \mathrm{f}$

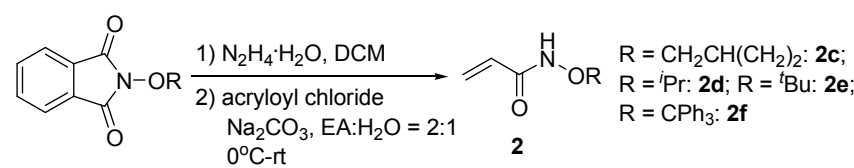

$N$-alkoxyphthalimides were prepared according to the literature procedure. ${ }^{[12-14]}$

In a suitable round-bottom flask, $N$-alkoxyphthalimide (1 equiv) was dissolved in DCM $(0.25 \mathrm{M})$. Then hydrazine monohydrate (4 equiv) was added. After stirring at room temperature overnight, the reaction mixture was filtered through a pad of Celite and the filter cake was washed with DCM. The filtrate was concentrated under reduced pressure. The resulting residue was used in the next step without further purification.

The product obtained above was added to a biphasic mixture of $\mathrm{Na}_{2} \mathrm{CO}_{3}$ (1.2 equiv) in a 2:1 mixture of EtOAc: $\mathrm{H}_{2} \mathrm{O}$. After the resulting mixture was cooled to $0{ }^{\circ} \mathrm{C}$, acryloyl chloride ( 1 equiv) was added dropwise. The reaction was allowed to warm to room temperature and stirred for 4 hours. Afterwards, the reaction was quenched with water, and the aqueous phase was extracted with EtOAc. The organic phase was combined and dried over $\mathrm{Na}_{2} \mathrm{SO}_{4}$, filtered and evaporated under reduced pressure. The crude product was purified by by flash column chromatography on silica gel or recrystalized from PE/EA.

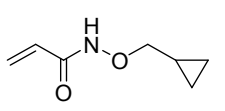

2c

Thick oil, $85 \%$ yield $(0.60 \mathrm{~g})$ from corresponding $N$-alkoxyphthalimide (5 mmol, 1.09 g, 1 equiv); ${ }^{1}$ H NMR (400 MHz, MeOD-d 4 ): $\delta 6.30$ (d, $\left.J=17.2 \mathrm{~Hz}, 1 \mathrm{H}\right), 6.11(\mathrm{dd}, J$ $=16.8 \mathrm{~Hz}, 10.4 \mathrm{~Hz}, 1 \mathrm{H}), 5.72(\mathrm{~d}, J=10.8 \mathrm{~Hz}, 1 \mathrm{H}), 3.69(\mathrm{~d}, J=7.2 \mathrm{~Hz}, 2 \mathrm{H})$, 1.19-1.01 (m, 1H), 0.60-0.56 (m, 2H), 0.31-0.27 (m, 2H); ${ }^{13}$ C NMR (100 MHz, MeOD-d $\left.{ }_{4}\right): \delta 163.7,127.5,126.4,80.7,8.9,2.5$; IR (neat, $\mathbf{~ c m}^{-1}$ ): $v 1656,1424,1043$; MS (m/z, ESI): 142; HRMS calculated for $\mathrm{C}_{7} \mathrm{H}_{12} \mathrm{NO}_{2}(\mathrm{M}+\mathrm{H})^{+}$: 142.0863 ; Found: $142.0863 ; \mathbf{R}_{\mathbf{f}}=0.3(\mathrm{EA}: \mathrm{PE}=1: 1)$

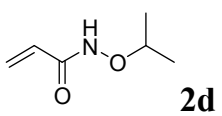

White solid, $79 \%$ yield $(0.51 \mathrm{~g})$ from corresponding $\mathrm{N}$-alkoxyphthalimide $(5 \mathrm{mmol}$, 
1.03 g, 1 equiv); m.p: $75-76{ }^{\circ} \mathrm{C} ;{ }^{1} \mathbf{H}$ NMR (400 MHz, DMSO-d 6 ): $\delta 10.97$ (s, 1H), 6.18-6.04 (m, 2H), 5.69 (dd, $J=9.6 \mathrm{~Hz}, 2.4 \mathrm{~Hz}, 1 \mathrm{H}), 3.97$ (m, 1H), 1.10(d, $J=6.4 \mathrm{~Hz}$, 6H); ${ }^{13}$ C NMR (100 MHz, DMSO-d 6 ): $\delta 162.2,128.6,125.9,76.6,20.6$; IR (neat, $\left.\mathbf{c m}^{-1}\right): v$ 2975, 2930, 1657, 1411, 1376, 1045; MS (m/z, ESI): 130, 129, 121; HRMS calculated for $\mathrm{C}_{6} \mathrm{H}_{12} \mathrm{NO}_{2}(\mathrm{M}+\mathrm{H})^{+}: 130.0863$; Found: $130.0863 ; \mathbf{R}_{\mathbf{f}}=0.3(\mathrm{EA}: \mathrm{PE}=$ $1: 2)$.

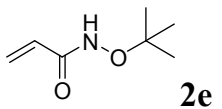

White solid, $80 \%$ yield $(0.57 \mathrm{~g})$ from corresponding $\mathrm{N}$-alkoxyphthalimide $(5 \mathrm{mmol}$, 1.10 g, 1 equiv); m.p: $58-59{ }^{\circ} \mathrm{C}$; ${ }^{1} \mathbf{H}$ NMR (400 MHz, DMSO-d $)$ ): $\delta 10.58(\mathrm{~s}, 1 \mathrm{H})$, 6.16-6.14 (m, 2H), 5.63 (dd, $J=7.6 \mathrm{~Hz}, 4.4 \mathrm{~Hz}, 1 \mathrm{H}), 1.16(\mathrm{~s}, 9 \mathrm{H}) ;{ }^{13} \mathrm{C}$ NMR (100 MHz, $\mathbf{C D C l}_{3}$ ): $\delta$ 165.1, 128.1, 126.7, 82.2, 26.3; IR (neat, $\mathbf{c m}^{-1}$ ): v 3466, 2980, 1667, 953; MS (m/z, EI): 143, 87, 57; HRMS calculated for: $\mathrm{C}_{7} \mathrm{H}_{13} \mathrm{NO}_{2}\left(\mathrm{M}^{+}\right)$: 143.0946; Found: $143.0947 ; \mathbf{R}_{\mathbf{f}}=0.3(\mathrm{EA}: \mathrm{PE}=1: 2)$

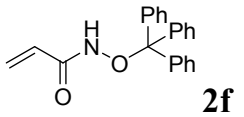

White solid, 78\% yield (1.28 g) from corresponding $\mathrm{N}$-alkoxyphthalimide (5 mmol, 2.03 g, 1 equiv); m.p: $138-139{ }^{\circ} \mathrm{C}$; The NMR spectra indicate this compound exists as mixture of tautomers (amide and imidic acid) in solution. There are no other significant impurities in this compound according to the elemental analysis. ${ }^{\mathbf{1}} \mathbf{H}$ NMR (400 MHz, DMSO-d $\left.{ }_{6}\right): \delta 10.52(\mathrm{~s}, 1 \mathrm{H}), 7.33-7.26(\mathrm{~m}, 15 \mathrm{H}), 6.03(\mathrm{dd}, J=17.2 \mathrm{~Hz}$, $10.4 \mathrm{~Hz}, 1 \mathrm{H}), 5.89(\mathrm{~d}, J=17.2 \mathrm{~Hz}, 1 \mathrm{H}), 5.28(\mathrm{~d}, J=10.0 \mathrm{~Hz}, 1 \mathrm{H}) ;{ }^{13} \mathbf{C}$ NMR $(\mathbf{1 0 0}$ MHz, $\left.\mathbf{C D C l}_{3}\right): \delta 168.7,155.6,143.8,141.8,141.0,129.1,128.1,126.7,127.4,126.1$, 124.9, 121.0, 93.9; IR (neat, $\mathbf{c m}^{-1}$ ): $v$ 1658, 1630, 1511, 1445, 751; Elemental analysis calculated for $\mathrm{C}_{22} \mathrm{H}_{19} \mathrm{NO}_{2}$ : C: $80.22 \%, \mathrm{H}: 5.81 \%, \mathrm{~N}: 4.25 \%$; Measured: $\mathrm{C}$ : 79.98\%, H: $5.78 \%$, N: 4.25\%; $\mathbf{R}_{\mathbf{f}}=0.2(\mathrm{EA}: \mathrm{PE}=1: 2)$

\section{Preparation of substrate $2 \mathrm{k}$}

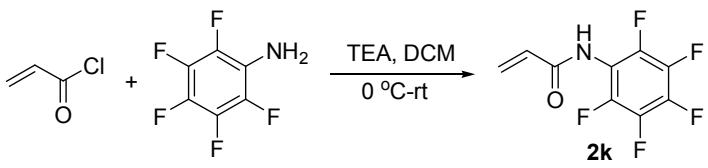

Pentafluoroaniline (1.83 g, $10 \mathrm{mmol}, 1$ equiv, ) and triethylamine $(1.65 \mathrm{~mL}, 12$ mmol, 1.2 equiv) was dissolved in DCM $(30 \mathrm{~mL})$. After the resulting solution was cooled to $0{ }^{\circ} \mathrm{C}$, acryloyl chloride $(0.91 \mathrm{~g}, 10 \mathrm{mmol}, 1$ equiv) was added dropwise. The reaction mixture was allowed to warm to room temperature and stirred overnight. Afterwards, the reaction was quenched by water and diluted with DCM. The organic phase was washed by water twice, dried over $\mathrm{Na}_{2} \mathrm{SO}_{4}$, filtered and evaporated under reduced pressure. The crude product was purified by flash column chromatography on silica gel to give desired products as white solid $(0.59 \mathrm{~g}, 25 \%)$. $2 \mathbf{k}$ is a known compound and all data were in agreement with those reported. ${ }^{[15]}$ 


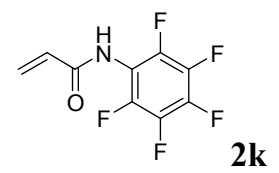

2k: 25\% yield; m.p: $93-95{ }^{\circ} \mathrm{C}$; ${ }^{1} \mathbf{H}$ NMR (400 MHz, $\left.\mathbf{C D C l}_{3}\right): \delta 7.80$ (s, 1H), $6.44(\mathrm{dd}$, $J=17.2 \mathrm{~Hz}, 1.2 \mathrm{~Hz}, 1 \mathrm{H}), 6.32(\mathrm{dd}, J=17.2 \mathrm{~Hz}, 10.0 \mathrm{~Hz}, 1 \mathrm{H}), 5.85(\mathrm{dd}, J=10.0 \mathrm{~Hz}$, $1.2 \mathrm{~Hz}, 1 \mathrm{H}) ;{ }^{13} \mathbf{C}$ NMR (100 $\left.\mathbf{~ M H z}, \mathbf{C D C l}_{3}\right)$ : The carbon spectra are complicated to find all ${ }^{19} \mathrm{~F}$-coupled carbons in pentafluoro phenyl group. $\delta 164.2,144.3-144.1(\mathrm{~m})$, 141.8-141.4 (m), 139.1-138.8 (m), 136.5-136.3 (m), 129.8, 128.8, $111.5\left(\mathrm{t}, J_{\mathrm{C}-\mathrm{F}}=15.1\right.$ $\mathrm{Hz}) ;{ }^{19} \mathrm{~F}$ NMR $\left(376 \mathrm{M}, \mathrm{CDCl}_{3}\right): \delta-144.5(\mathrm{~d}, J=16 \mathrm{~Hz}),-156.2(\mathrm{t}, J=18 \mathrm{~Hz}),-162.2(\mathrm{t}$, $J=18 \mathrm{~Hz}$ ); IR (neat, $\left.\mathbf{c m}^{-1}\right): v$ 3217, 2923, 2853, 1685, 1520, 1300, 1211; MS (m/z, ESI): 238; HRMS calculated for $\mathrm{C}_{9} \mathrm{H}_{4} \mathrm{~F}_{5} \mathrm{NO}(\mathrm{M}+\mathrm{H})^{+}: 238.0286$; Found: 238.0287; $\mathbf{R}_{\mathbf{f}}$ $=0.2(\mathrm{PE}: \mathrm{EA}=6: 1)$.

\section{Preparation of substrate 12}

$$
\overbrace{\mathrm{O}}+\mathrm{NH}_{2} \mathrm{OH} \cdot \mathrm{HCl} \frac{1) \mathrm{Na}_{2} \mathrm{CO}_{3}, \mathrm{MeOH}, \mathrm{rt}}{2) \mathrm{Et}_{3} \mathrm{~N}, \mathrm{PivCl}, \mathrm{DCM}} \overbrace{0^{\circ} \mathrm{C}-\mathrm{rt}}^{\mathrm{N}}
$$

In a suitable round-bottom flask, $\mathrm{Na}_{2} \mathrm{CO}_{3}(14 \mathrm{mmol}, 1.50 \mathrm{~g}, 1.4$ equiv) was added to the solution of hydroxylamine hydrochloride (14 mmol, $0.972 \mathrm{~g}, 1.4$ equiv) in $\mathrm{MeOH}(30 \mathrm{~mL})$. The resulting mixture was stirred for ten minutes, and then methyl vinyl ketone (10 mmol, $0.84 \mathrm{~mL}, 1$ equiv) was added. After the reaction mixture was stirred for $4 \mathrm{~h}$, the solvent was concentrated. The crude product was dissolved in DCM (30 mL), and $\mathrm{Et}_{3} \mathrm{~N}$ ( $25 \mathrm{mmol}, 3.5 \mathrm{~mL}, 2.5$ equiv) was added. After the resulting solution was cooled to $0{ }^{\circ} \mathrm{C}$, pivaloyl chloride $(0.91 \mathrm{~g}, 10 \mathrm{mmol}, 1$ equiv) was added dropwise. The reaction mixture was allowed to warm to room temperature and stirred overnight. Afterwards, the reaction was quenched by water. The organic phase was washed by water three times, dried over $\mathrm{Na}_{2} \mathrm{SO}_{4}$, filtered and evaporated under reduced pressure. The crude product was purified by flash column chromatography on silica gel to give the desired product as colorless oil $(0.3 \mathrm{~g}, 18 \%)$, which was stored at $0{ }^{\circ} \mathrm{C}$.

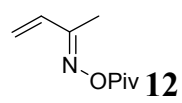

12: ${ }^{1}$ H NMR (400 MHz, $\left.\mathbf{C D C l}_{3}\right): \delta 6.41(\mathrm{dd}, J=18.0 \mathrm{~Hz}, 11.2 \mathrm{~Hz}, 1 \mathrm{H}), 5.62(\mathrm{~d}, J=$ $17.6 \mathrm{~Hz}, 1 \mathrm{H}), 5.47(\mathrm{~d}, J=10.8 \mathrm{~Hz}, 1 \mathrm{H}), 1.92(\mathrm{~s}, 3 \mathrm{H}), 1.11(\mathrm{~s}, 9 \mathrm{H}) ;{ }^{13} \mathbf{C}$ NMR (100 MHz, $\mathbf{C D C l}_{3}$ ): $\delta 174.4,162.3,132.8,122.6,38.3,26.8,10.4$; IR (neat, $\mathbf{~ c m}^{-1}$ ): $v$ 2974, 1759, 1480, 1370, 1100 ; MS (m/z, ESI): 170; HRMS calculated for $\mathrm{C}_{9} \mathrm{H}_{16} \mathrm{NO}_{2}$ $\left(\mathrm{M}+\mathrm{H}^{+}\right): 170.1176$; Found: $170.1177 ; \mathbf{R}_{\mathbf{f}}=0.5(\mathrm{EA}: \mathrm{PE}=1: 10)$.

\section{Synthesis of $o$-tyrosine derivatives}

\subsection{Optimization Studies (Table S1)}

$N$-phenoxyacetamide (1a, $18.2 \mathrm{mg}, 0.12 \mathrm{mmol}$ ), $N$-methoxyacrylamide (2a, 10.1 $\mathrm{mg}, 0.1 \mathrm{mmol})$, [Cp* $\left.\mathrm{RhCl}_{2}\right]_{2}(1.6 \mathrm{mg}, 2.5 \mathrm{~mol} \%)$, and base were weighted in a $10 \mathrm{~mL}$ Schlenk tube equipped with a stir bar. The reaction tube was evacuated and refilled 
with nitrogen three times and then $\mathrm{CH}_{3} \mathrm{CN}(0.5 \mathrm{~mL})$ was added. The reaction mixture was stirred at room temperature under $\mathrm{N}_{2}$ until completion. Afterwards, the reaction mixture was filtered through a pad of Celite and the filter cake was washed with $\mathrm{MeOH}$. The combined filtration was concentrated and ${ }^{1} \mathrm{H}$ NMR was taken using trimethoxybenzene $(5.6 \mathrm{mg})$ as the internal standard.

Table S1. Optimization of reaction conditions ${ }^{a}$

\begin{tabular}{|c|c|c|c|c|c|c|}
\hline \multirow{2}{*}{ Entry } & \multirow{2}{*}{ Sovlent } & \multirow{2}{*}{ Base (equiv) } & \multirow{2}{*}{ Time $(h)$} & \multicolumn{3}{|c|}{ Yield $(\%)^{c}$} \\
\hline & & & & $3 \mathbf{a a}$ & 4aa & $5 \mathbf{a a}$ \\
\hline 1 & $\mathrm{EtOH}$ & $\mathrm{CsOAc}(0.5)$ & 24 & 36 & 26 & 26 \\
\hline 2 & $\mathrm{CH}_{3} \mathrm{CN}$ & $\mathrm{Cs}_{2} \mathrm{CO}_{3}(0.5)$ & 90 & 50 & 3 & ND \\
\hline 3 & $\mathrm{CH}_{3} \mathrm{CN}$ & KOAc (0.5) & 22 & 57 & 5 & 15 \\
\hline 4 & $\mathrm{CH}_{3} \mathrm{CN}$ & $\mathrm{K}_{2} \mathrm{CO}_{3}(0.5)$ & 92 & 52 & 2 & ND \\
\hline 5 & $\mathrm{CH}_{3} \mathrm{CN}$ & $\mathrm{CsOAc}(0.5)$ & 14 & 69 & 10 & 23 \\
\hline 6 & $\mathrm{CH}_{3} \mathrm{CN}$ & $\mathrm{K}_{3} \mathrm{PO}_{4}(0.5)$ & 48 & 78 & ND & ND \\
\hline $7^{b}$ & $\mathrm{CH}_{3} \mathrm{CN}$ & $\mathbf{K}_{3} \mathbf{P O}_{4}(0.5)$ & 20 & $93\left(87^{d}\right)$ & ND & ND \\
\hline $8^{b}$ & $\mathrm{CH}_{3} \mathrm{CN}$ & $\mathrm{K}_{2} \mathrm{CO}_{3}(0.5)$ & 40 & 84 & ND & $\mathrm{ND}$ \\
\hline $9^{b}$ & $\mathrm{CH}_{3} \mathrm{CN}$ & $\mathrm{Cs}_{2} \mathrm{CO}_{3}(0.5)$ & 24 & 66 & ND & 10 \\
\hline $10^{b}$ & $\mathrm{CH}_{3} \mathrm{CN}$ & $\mathrm{NaOAc}(0.5)$ & 24 & 45 & ND & 21 \\
\hline $11^{b}$ & $\mathrm{CH}_{3} \mathrm{CN}$ & $\mathrm{CsOAc}(0.5)$ & 92 & 35 & ND & 28 \\
\hline $12^{b}$ & THF & $\mathrm{K}_{3} \mathrm{PO}_{4}(0.5)$ & 24 & 63 & ND & 5 \\
\hline $13^{b}$ & 1,4-dioxane & $\mathrm{K}_{3} \mathrm{PO}_{4}(0.5)$ & 90 & 42 & ND & 9 \\
\hline $14^{b}$ & DCE & $\mathrm{K}_{3} \mathrm{PO}_{4}(0.5)$ & 120 & 20 & 7 & ND \\
\hline $15^{b}$ & $\mathrm{CH}_{3} \mathrm{CN}$ & $\mathrm{K}_{3} \mathrm{PO}_{4}(0.25)$ & 20 & 73 & ND & 8 \\
\hline $16^{b}$ & $\mathrm{CH}_{3} \mathrm{CN}$ & $\mathrm{K}_{3} \mathrm{PO}_{4}(1)$ & 20 & 87 & ND & 6 \\
\hline
\end{tabular}

${ }^{a}$ Reaction condition: 1a $\left(0.12 \mathrm{mmol}, 1.2\right.$ equiv), $\mathbf{2 a}\left(0.1 \mathrm{mmol}, 1 \text { equiv), [Cp* } \mathrm{RhCl}_{2}\right]_{2}(2.5 \mathrm{~mol} \%)$, solvent $(0.4 \mathrm{M})$, rt. ${ }^{b}$ additive: $50 \mathrm{mg} 4 \AA \mathrm{MS} .{ }^{c}{ }^{1} \mathrm{H}$ HMR yield, 1,3,5-trimethoxybenzene as internal standard. ${ }^{d}$ isolated yield.

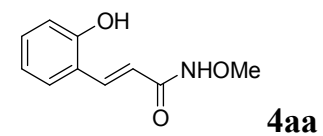

m.p: 156-157 ${ }^{\circ} \mathrm{C} ;{ }^{1} \mathbf{H}$ NMR (400 MHz, MeOD-d 4$): \delta 7.86(\mathrm{~d}, J=16.0 \mathrm{~Hz}, 1 \mathrm{H}), 7.43$ $(\mathrm{d}, J=7.2 \mathrm{~Hz}, 1 \mathrm{H}), 7.18(\mathrm{t}, J=7.6 \mathrm{~Hz}, 1 \mathrm{H}), 6.85-6.81(\mathrm{~m}, 2 \mathrm{H}), 6.58(\mathrm{~d}, J=16.0 \mathrm{~Hz}$, 1H), 3.75 (s, 3H); ${ }^{13}$ C NMR (100 MHz, MeOD-d 4$): \delta$ 167.1, 158.2, 139.0, 132.1, 130.2, 122.9, 120.7, 117.7, 117.0, 64.5; IR (neat, $\mathbf{c m}^{-1}$ ): $v$ 3175, 2967, 1642, 1600, 1456, 1253, 983; MS (m/z, ESI): 194, 152, 147; HRMS calculated for $\mathrm{C}_{10} \mathrm{H}_{12} \mathrm{NO}_{3}$ $(\mathrm{M}+\mathrm{H})^{+}:$194.0812; Found: 194.0807; $\mathbf{R}_{\mathbf{f}}=0.1(\mathrm{EA}: \mathrm{PE}=1: 1)$.

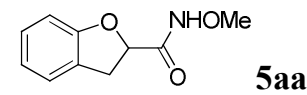

The title compound was obtained as white solid; m.p: $63-64{ }^{\circ} \mathrm{C} ;{ }^{1} \mathbf{H}$ NMR (400 MHz, 
$\left.\mathbf{C D C l}_{3}\right): \delta 9.06(\mathrm{~s}, 1 \mathrm{H}), 7.21-7.14(\mathrm{~m}, 2 \mathrm{H}), 6.93(\mathrm{t}, J=7.6 \mathrm{~Hz}, 1 \mathrm{H}), 6.84(\mathrm{~d}, J=8.0$ $\mathrm{Hz}, 1 \mathrm{H}), 5.20$ (dd, $J=11.2 \mathrm{~Hz}, 6.8 \mathrm{~Hz}, 1 \mathrm{H}), 3.79$ (s, 3H), 3.61 (dd, $J=16.0 \mathrm{~Hz}, 11.2$ $\mathrm{Hz}, 1 \mathrm{H}), 3.46(\mathrm{dd}, J=16.0 \mathrm{~Hz}, 6.8 \mathrm{~Hz}, 1 \mathrm{H}) ;{ }^{13} \mathbf{C}$ NMR (100 MHz, $\left.\mathbf{C D C l}_{3}\right): \delta 168.9$, 158.2, 128.5, 125.4, 125.1, 122.1, 109.8, 80.1, 64.8, 34.0; IR (neat, $\mathbf{c m}^{-1}$ ): $v$ 3303, 1662, 1594, 1480, 1458, 1231, 746; MS (m/z, ESI): 194, 152, 121, 110; HRMS calculated for $\mathrm{C}_{10} \mathrm{H}_{12} \mathrm{NO}_{3}(\mathrm{M}+\mathrm{H})^{+}:$194.0812; Found: 194.1811; $\mathbf{R}_{\mathbf{f}}=0.4$ (DCM:EA = 10:1).

\subsection{General Procedure}

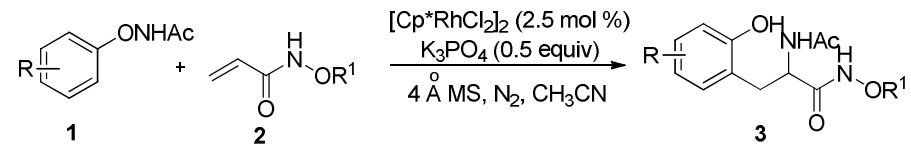

$N$-aryloxyacetamide 1 ( $0.24 \mathrm{mmol}, 1.2$ equiv), $N$-alkoxyacrylamides 2 ( $0.2 \mathrm{mmol}$, 1 equiv), [Cp* $\left.\mathrm{RhCl}_{2}\right]_{2}(3.2 \mathrm{mg}, 2.5 \mathrm{~mol} \%), \mathrm{K}_{3} \mathrm{PO}_{4}(0.1 \mathrm{mmol}, 0.5$ equiv) and $4 \AA \mathrm{MS}$ $(100 \mathrm{mg})$ were weighted in a $10 \mathrm{~mL}$ Schlenk tube equipped with a stir bar. The reaction tube was evacuated and refilled with nitrogen three times and then $\mathrm{CH}_{3} \mathrm{CN}$ $(0.4 \mathrm{M})$ was added. The reaction was stirred at room temperature under $\mathrm{N}_{2}$ for 20-96 h. Afterwards, the reaction mixture was filtered through a pad of Celite and the filter cake was washed with $\mathrm{MeOH}$. Silica gel was added to the filtrate and volatiles were evaporated under reduced pressure. The purification was performed by flash column chromatography on silica gel (see below for specific eluents).

\subsection{Characterization of products $3,4,5$}

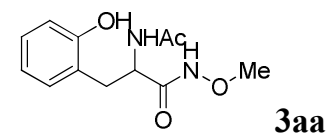

Yellow solid, $87 \%$ yield (44 mg) from $N$-methoxyacrylamide (20.2 mg, $0.2 \mathrm{mmol}, 1$ equiv); m.p: $164-165{ }^{\circ} \mathrm{C}$; ${ }^{1} \mathbf{H}$ NMR (400 MHz, DMSO-d $\left.{ }_{6}\right): \delta 11.13$ (s, 1H), 9.39 (s, $1 \mathrm{H}), 8.09(\mathrm{~d}, J=8.0 \mathrm{~Hz}, 1 \mathrm{H}), 7.02-6.98(\mathrm{~m}, 2 \mathrm{H}), 6.76(\mathrm{~d}, J=7.6 \mathrm{~Hz}, 1 \mathrm{H}), 6.67(\mathrm{t}, J=$ $7.2 \mathrm{~Hz}, 1 \mathrm{H}), 4.32$ (dd, $J=14.8 \mathrm{~Hz}, 8.0 \mathrm{~Hz}, 1 \mathrm{H}), 3.44$ (s, 3H), 2.87 (dd, $J=13.2 \mathrm{~Hz}$, $6.4 \mathrm{~Hz}, 1 \mathrm{H}), 2.72$ (dd, $J=13.2 \mathrm{~Hz}, 8.4 \mathrm{~Hz}, 1 \mathrm{H}), 1.76$ (s, 3H); ${ }^{13}$ C NMR (100 MHz, MeOD-d $\left._{4}\right): \delta 173.1,170.5,156.7,132.3,129.3,124.1,120.5,115.9,64.2,52.8,33.8$, 22.4; IR (neat, $\mathbf{~ c m}^{-1}$ ): $v$ 3344, 3156, 1658, 1601, 1553, 1477, 1436, 1257, 762; MS (m/z, ESI): 253, 206, 178; HRMS calculated for $\mathrm{C}_{12} \mathrm{H}_{17} \mathrm{~N}_{2} \mathrm{O}_{4}(\mathrm{M}+\mathrm{H})^{+}:$: 253.1183; Found: $253.1190 ; \mathbf{R}_{\mathbf{f}}=0.1$ (EA).

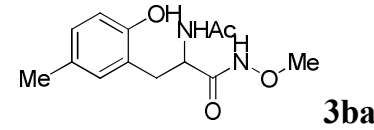

Yellow solid, 69\% yield (37 mg) from $N$-methoxyacrylamide $(20.2 \mathrm{mg}, 0.2 \mathrm{mmol}, 1$ equiv); m.p: $154-155{ }^{\circ} \mathrm{C} ;{ }^{1}$ H NMR (400 MHz, MeOD-d $\left.{ }_{4}\right): \delta 6.87-6.85$ (m, 2H), 6.67 $(\mathrm{d}, J=8.0 \mathrm{~Hz}, 1 \mathrm{H}), 4.44(\mathrm{t}, J=7.2 \mathrm{~Hz}, 1 \mathrm{H}), 3.51(\mathrm{~s}, 3 \mathrm{H}), 2.95(\mathrm{~d}, J=7.2 \mathrm{~Hz}, 2 \mathrm{H})$, 2.18 (s, 3H), 1.92 (s, 3H); ${ }^{13}$ C NMR (100 MHz, MeOD-d 4$): \delta 173.1,170.5,154.3$, 132.9, 129.6, 129.57, 123.8, 115.8, 64.2, 53.2, 33.7, 22.4, 20.5; IR (neat, $\mathbf{c m}^{-1}$ ): v 3345, 3163, 1661, 1610, 1502, 1265, 820; MS (m/z, ESI): 267, 220, 192; HRMS calculated for $\mathrm{C}_{13} \mathrm{H}_{19} \mathrm{~N}_{2} \mathrm{O}_{4}(\mathrm{M}+\mathrm{H})^{+}: 267.1339$; Found: 267.1345; $\mathbf{R}_{\mathbf{f}}=0.2$ (EA). 


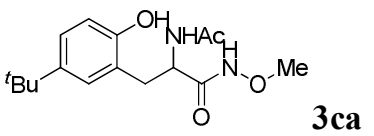

Yellow solid, 71\% yield (44 mg) from $N$-methoxyacrylamide $(20.2 \mathrm{mg}, 0.2 \mathrm{mmol}, 1$ equiv); m.p: $148-149{ }^{\circ} \mathrm{C} ;{ }^{1}$ H NMR (400 MHz, MeOD-d 4 ): $\delta$ 7.10-7.08 (m, 2H), 6.70 $(\mathrm{d}, J=8.4 \mathrm{~Hz}, 1 \mathrm{H}), 4.47(\mathrm{t}, J=7.6 \mathrm{~Hz}, 1 \mathrm{H}), 3.53(\mathrm{~s}, 3 \mathrm{H}), 2.98(\mathrm{dd}, J=7.2 \mathrm{~Hz}, 3.6 \mathrm{~Hz}$, 2H), 1.91 (s, 3H), 1.25 (s, 9H); ${ }^{13}$ C NMR (100 MHz, MeOD-d 4$): \delta 173.0,170.5$, $154.2,143.2,129.3,125.9,123.4,115.4,64.3,53.1,34.7,34.0,32.0,22.5$; IR (neat, $\left.\mathbf{c m}^{-1}\right)$ : v 3173, 1640, 1537, 1508, 1462, 1272, 823; MS (m/z, ESI): 309, 297, 262; HRMS calculated for $\mathrm{C}_{16} \mathrm{H}_{25} \mathrm{~N}_{2} \mathrm{O}_{4}(\mathrm{M}+\mathrm{H})^{+}: 309.1809$; Found: $309.1812 ; \mathbf{R}_{\mathbf{f}}=0.2$ (EA).

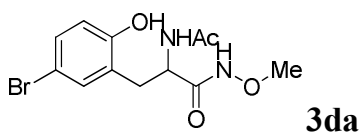

Yellow solid, $88 \%$ yield $(58 \mathrm{mg}$ ) from $N$-methoxyacrylamide $(20.2 \mathrm{mg}, 0.2 \mathrm{mmol}, 1$ equiv); m.p: $145-146{ }^{\circ} \mathrm{C} ;{ }^{1} \mathbf{H}$ NMR (400M Hz, MeOD-d 4 ): $\delta$ 7.21-7.16 (m, 2H), 6.71 $(\mathrm{d}, J=8.4 \mathrm{~Hz}, 1 \mathrm{H}), 4.48(\mathrm{t}, J=7.6 \mathrm{~Hz}, 1 \mathrm{H}), 3.56(\mathrm{~s}, 3 \mathrm{H}), 3.02-2.89(\mathrm{~m}, 2 \mathrm{H}), 1.92(\mathrm{~s}$, $3 \mathrm{H}) ;{ }^{13}$ C NMR (100M, MeOD-d 4$): \delta$ 173.0, 170.1, 156.2, 134.8, 131.9, 126.8, 117.7, 111.8, 64.2, 52.3, 33.5, 22.4; IR (neat, $\mathbf{~ c m}^{-1}$ ): $v$ 3354, 3188, 1665, 1620, 1595, 1548, 1515, 1264, 906, 821, 646; MS (m/z, ESI): 331, 286, 284; HRMS calculated for $\mathrm{C}_{16} \mathrm{H}_{16} \mathrm{BrN}_{2} \mathrm{O}_{4}(\mathrm{M}+\mathrm{H})^{+}: 331.0288$; Found: 331.0291; $\mathbf{R}_{\mathbf{f}}=0.2$ (EA).

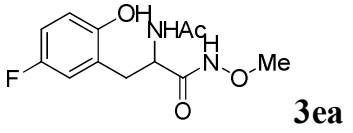

Yellow solid, 46\% yield (25 mg) from $N$-methoxyacrylamide (20.2 mg, $0.2 \mathrm{mmol}, 1$ equiv); m.p: $150-151{ }^{\circ} \mathrm{C} ;{ }^{1} \mathbf{H}$ NMR (400 MHz, MeOD-d $\left.{ }_{4}\right): \delta 6.84-6.72(\mathrm{~m}, 3 \mathrm{H}), 4.49$ $(\mathrm{t}, J=7.6 \mathrm{~Hz}, 1 \mathrm{H}), 3.56(\mathrm{~s}, 3 \mathrm{H}), 3.03-2.90(\mathrm{~m}, 2 \mathrm{H}), 1.92(\mathrm{~s}, 3 \mathrm{H}) ;{ }^{13} \mathrm{C}$ NMR (100 MHz, MeOD-d 4$): \delta 173.1,170.3,157.5\left(\mathrm{~d}, J_{\mathrm{C}-\mathrm{F}}=233.8 \mathrm{~Hz}\right), 153.0,125.8\left(\mathrm{~d}, J_{\mathrm{C}-\mathrm{F}}=7.6 \mathrm{~Hz}\right)$, $118.3\left(\mathrm{~d}, J_{\mathrm{C}-\mathrm{F}}=22.8 \mathrm{~Hz}\right), 116.5\left(\mathrm{~d}, J_{\mathrm{C}-\mathrm{F}}=8.3\right), 115.1\left(\mathrm{~d}, J_{\mathrm{C}-\mathrm{F}}=22.8 \mathrm{~Hz}\right), 64.2,52.5$, 33.7, 22.4; ${ }^{19} \mathrm{~F}$ NMR (376 MHz, MeOD-d 4 ) $\delta$-129.3; IR (neat, cm $^{-1}$ ): $v$ 3247, 1711, 1676, 1549, 1442, 1211, 816; MS (m/z, ESI): 271, 268, 225, 224, 196; HRMS calculated for $\mathrm{C}_{12} \mathrm{H}_{16} \mathrm{FN}_{2} \mathrm{O}_{4}(\mathrm{M}+\mathrm{H})^{+}: 271.1098$; Found: 271.1094; $\mathbf{R}_{\mathbf{f}}=0.2(\mathrm{EA})$.

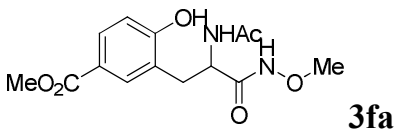

Yellow solid, 73\% yield (45 mg) from $N$-methoxyacrylamide $(20.2 \mathrm{mg}, 0.2 \mathrm{mmol}, 1$ equiv); m.p: $180-181{ }^{\circ} \mathrm{C} ;{ }^{1} \mathbf{H}$ NMR (400 MHz, MeOD-d M): $\delta$ 7.79-7.75 (m, 2H), 6.83 $(\mathrm{d}, J=8.4 \mathrm{~Hz}, 1 \mathrm{H}), 4.52(\mathrm{t}, J=7.6 \mathrm{~Hz}, 1 \mathrm{H}), 3.82(\mathrm{~s}, 3 \mathrm{H}), 3.53(\mathrm{~s}, 3 \mathrm{H}), 3.07(\mathrm{dd}, J=$ $13.2 \mathrm{~Hz}, 7.2 \mathrm{~Hz}, 1 \mathrm{H}), 2.96(\mathrm{dd}, J=13.2 \mathrm{~Hz}, 7.6 \mathrm{~Hz}, 1 \mathrm{H}), 1.91(\mathrm{~s}, 3 \mathrm{H}) ;{ }^{13}$ C NMR (100 MHz, MeOD-d $\left.{ }_{4}\right): \delta 173.0,170.2,168.6,161.7,134.3,131.5,124.5,122.1,115.7$, 64.2, 52.2, 33.7, 22.4; IR (neat, $\mathbf{~ c m}^{-1}$ ): $v$ 3362, 3307, 1713, 1668, 1607, 1279, 772; MS (m/z, ESI): 311, 264; HRMS calculated for $\mathrm{C}_{14} \mathrm{H}_{19} \mathrm{~N}_{2} \mathrm{O}_{6}(\mathrm{M}+\mathrm{H})^{+}$: 311.1238 ; Found: $311.1242 ; \mathbf{R}_{\mathbf{f}}=0.2$ (EA). 


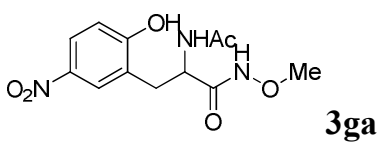

Yellow solid, $46 \%$ yield $(27 \mathrm{mg}$ ) from $N$-methoxyacrylamide $(20.2 \mathrm{mg}, 0.2 \mathrm{mmol}, 1$ equiv); m.p: $176-177{ }^{\circ} \mathrm{C}$; ${ }^{1}$ H NMR (400 MHz, MeOD-d 4 ): $\delta$ 8.06-8.01 (m, 2H), 6.90 $(\mathrm{d}, J=8.8 \mathrm{~Hz}, 1 \mathrm{H}), 4.57(\mathrm{t}, J=7.6 \mathrm{~Hz}, 1 \mathrm{H}), 3.56(\mathrm{~s}, 3 \mathrm{H}), 3.13(\mathrm{dd}, J=13.6 \mathrm{~Hz}, 6.8$ $\mathrm{Hz}, 1 \mathrm{H}), 2.97$ (dd, $J=13.6 \mathrm{~Hz}, 8.4 \mathrm{~Hz}, 1 \mathrm{H}), 1.91(\mathrm{~s}, 3 \mathrm{H}) ;{ }^{13} \mathrm{C}$ NMR (100 MHz, MeOD-d 4 ): $\delta 173.1,170.0,163.3,141.4,128.2$, 125.7, 125.6, 115.8, 64.2, 51.8, 33.5, 22.4; IR (neat, $\mathbf{~ c m}^{-1}$ ): $v$ 3407, 1682, 1643, 1590, 1518, 1335, 920, 835; MS (m/z, EI): 297, 251, 191, 163, 133; HRMS calculated for $\mathrm{C}_{12} \mathrm{H}_{15} \mathrm{~N}_{3} \mathrm{O}_{6}\left(\mathrm{M}^{+}\right)$: 297.0961; Found: $297.0965 ; \mathbf{R}_{\mathbf{f}}=0.2(\mathrm{EA})$.

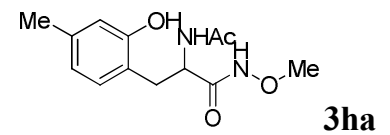

Yellow solid, $80 \%$ yield (43 mg) from $N$-methoxyacrylamide $(20.2 \mathrm{mg}, 0.2 \mathrm{mmol}, 1$ equiv); m.p: $150-151{ }^{\circ} \mathrm{C} ;{ }^{1} \mathbf{H}$ NMR (400 MHz, MeOD-d 4 ): $\delta 6.93(\mathrm{~d}, J=7.6 \mathrm{~Hz}, 1 \mathrm{H})$, $6.62(\mathrm{~s}, 1 \mathrm{H}), 6.56(\mathrm{~d}, J=7.6 \mathrm{~Hz}, 1 \mathrm{H}), 4.44(\mathrm{t}, J=7.2 \mathrm{~Hz}, 1 \mathrm{H}), 3.51(\mathrm{~s}, 3 \mathrm{H}), 2.96-2.93$ (m, 2H), 2.20 (s, 3H), 1.91 (s, 3H); ${ }^{13}$ C NMR (100 MHz, MeOD-d 4 ): $\delta$ 173.1, 170.6, 156.5, 139.3, 132.1, 121.2, 121.1, 116.6, 64.2, 53.2, 33.4, 22.4, 21.2; IR (neat, $\mathbf{~ c m}^{-1}$ ): $v 3231, \quad 3054, \quad 1670, \quad 1629, \quad 1582, \quad 1551, \quad 851, \quad 805 ; \quad \mathbf{M S} \quad(\mathbf{m} / \mathbf{z}$, ESI): 267, 220, 192; HRMS calculated for $\mathrm{C}_{13} \mathrm{H}_{19} \mathrm{~N}_{2} \mathrm{O}_{4}(\mathrm{M}+\mathrm{H})^{+}$: 267.1339; Found: $267.1341 ; \mathbf{R}_{\mathbf{f}}=0.2(\mathrm{EA})$.

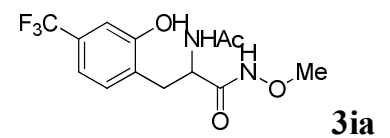

White solid, 55\% yield (35 mg) from $N$-methoxyacrylamide $(20.2 \mathrm{mg}, 0.2 \mathrm{mmol}, 1$ equiv); m.p: $182-183{ }^{\circ} \mathrm{C} ;{ }^{1} \mathbf{H}$ NMR (400 MHz, MeOD-d $)$ ): $\delta 7.23(\mathrm{~d}, J=7.2 \mathrm{~Hz}, 1 \mathrm{H})$, 7.03-7.02 (m, 2H), 4.55 (t, $J=7.2 \mathrm{~Hz}, 1 \mathrm{H}), 3.54(\mathrm{~s}, 3 \mathrm{H}), 3.10$ (dd, $J=13.2 \mathrm{~Hz}, 7.2 \mathrm{~Hz}$, 1H), 2.99 (dd, $J=13.2 \mathrm{~Hz}, 8.0 \mathrm{~Hz}, 1 \mathrm{H}), 1.91(\mathrm{~s}, 3 \mathrm{H}) ;{ }^{13} \mathbf{C}$ NMR (100 MHz, MeOD-d $_{4}$ ): $\delta 173.1,170.2,157.4,133.0,128.9,127.9$ (q, $\left.J_{C-F}=244.9 \mathrm{~Hz}\right), 116.8$, 116.7, 112.3, 64.2, 52.1, 33.7, 22.4; ${ }^{19} \mathrm{~F}$ NMR (376 MHz, MeOD-d 4 ) $\delta$-65.4; IR (neat, $\left.\mathbf{c m}^{-1}\right): v 3271,3324,1714,1674,1556,1486,1424,1330,922,870 ;$ MS (m/z, EI): 320, 274, 214, 204, 186, 103; HRMS calculated for $\mathrm{C}_{13} \mathrm{H}_{15} \mathrm{~F}_{3} \mathrm{~N}_{2} \mathrm{O}_{4}\left(\mathrm{M}^{+}\right)$: 320.0984; Found: 320.0982; $\mathbf{R}_{\mathbf{f}}=0.1$ (EA).

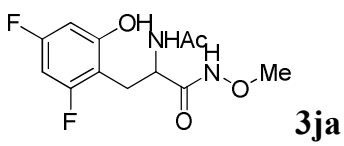

White solid, 76\% yield (66 mg) from $N$-methoxyacrylamide $(30.3 \mathrm{mg}, 0.3 \mathrm{mmol}, 1$ equiv); m.p: $178-179{ }^{\circ} \mathrm{C} ;{ }^{\mathbf{1}} \mathbf{H}$ NMR (400 MHz, MeOD-d $\left.{ }_{4}\right): \delta 6.40-6.33$ (m, 2H), 4.46 (t, $J=7.2 \mathrm{~Hz}, 1 \mathrm{H}), 3.59$ (s, 3H), 3.07 (dd, $J=13.2 \mathrm{~Hz}, 7.2 \mathrm{~Hz}, 1 \mathrm{H}), 2.92$ (dd, $J=13.2$ $\mathrm{Hz}, 7.6 \mathrm{~Hz}, 1 \mathrm{H}), 1.92$ (s, 3H); ${ }^{\mathbf{1 3}} \mathbf{C}$ NMR (100 MHz, MeOD-d $)$ ): $\delta$ 173.0, 170.2, 163.7 $\left(\mathrm{dd}, J_{\mathrm{C}-\mathrm{F}}=242.1,15.9 \mathrm{~Hz}\right), 159.3\left(\mathrm{~d}, J_{\mathrm{C}-\mathrm{F}}=13.7 \mathrm{~Hz}\right), 108.4\left(\mathrm{dd}, J_{\mathrm{C}-\mathrm{F}}=18.2,3.8 \mathrm{~Hz}\right)$, $99.3\left(\mathrm{~d}, J_{\mathrm{C}-\mathrm{F}}=23.6 \mathrm{~Hz}\right), 95.3\left(\mathrm{~d}, J_{\mathrm{C}-\mathrm{F}}=26.0 \mathrm{~Hz}\right), 64.1,52.0,26.1,22.4 ;{ }^{19} \mathrm{~F} \mathrm{NMR}$ (376 MHz, MeOD-d 4 ) $\delta$-115.5, -116.5; IR (neat, cm$^{-1}$ ): $v$ 3320, 3124, 1672, 1622, 
1544, 1502, 1460, 849; MS (m/z, ESI): 289, 242, 214, 172; HRMS calculated for $\mathrm{C}_{12} \mathrm{H}_{15} \mathrm{~F}_{2} \mathrm{~N}_{2} \mathrm{O}_{4}(\mathrm{M}+\mathrm{H})^{+}:$289.0994; Found: 289.0999; $\mathbf{R}_{\mathbf{f}}=0.2$ (EA).

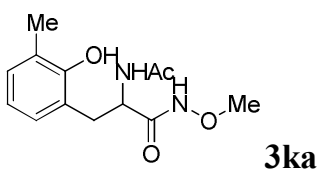

Yellow solid, 32\% yield (17 mg) from $N$-methoxyacrylamide $(20.2 \mathrm{mg}, 0.2 \mathrm{mmol}, 1$ equiv); m.p: $124-125{ }^{\circ} \mathrm{C} ;{ }^{1} \mathbf{H}$ NMR (400 MHz, MeOD-d 4 ): $\delta 6.97$ (d, $\left.J=7.2 \mathrm{~Hz}, 1 \mathrm{H}\right)$, $6.92(\mathrm{~d}, J=7.6 \mathrm{~Hz}, 1 \mathrm{H}), 6.69(\mathrm{t}, J=7.6 \mathrm{~Hz}, 1 \mathrm{H}), 4.44(\mathrm{t}, J=7.2 \mathrm{~Hz}, 1 \mathrm{H}), 3.51(\mathrm{~s}, 3 \mathrm{H})$, 3.06-2.96 (m, 2H), 2.22 (s, 3H), 1.93 (s, 3H); ${ }^{13}$ C NMR (100 MHz, MeOD-d 4 ): $\delta$ $173.1,170.4,154.5,130.8,130.0,125.8,124.7,120.9,64.2,53.1,34.0,22.4,16.7$; IR (neat, $\mathbf{c m}^{-1}$ ): $v$ 3381, 3203, 1686, 1602, 1485, 742; MS (m/z, ESI): 267, 220, 192; HRMS calculated for $\mathrm{C}_{13} \mathrm{H}_{19} \mathrm{~N}_{2} \mathrm{O}_{4}(\mathrm{M}+\mathrm{H})^{+}:$267.1339; Found: $267.1346 ; \mathbf{R}_{\mathbf{f}}=0.2$ (EA).

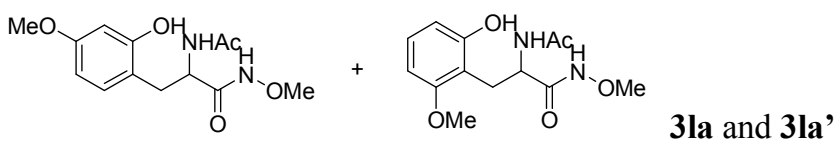

Yield 84\% (71 mg) from $N$-methoxyacrylamide (30.3 mg, $0.3 \mathrm{mmol}, 1$ equiv); 31 and 3I' are inseparable yellow solid, 31:3I' $=3.1: 1{ }^{1}{ }^{1} \mathbf{H}$ NMR (400 MHz, MeOD-d 4 ): $\delta$ $7.02(\mathrm{t}, J=8.0 \mathrm{~Hz}, 0.32 \mathrm{H}), 6.95(\mathrm{~d}, J=8.0 \mathrm{~Hz}, 1 \mathrm{H}), 6.45(\mathrm{t}, J=8.0 \mathrm{~Hz}, 0.64 \mathrm{H})$, 6.38-6.32 (m, 2H), $4.41(\mathrm{t}, J=7.6 \mathrm{~Hz}, 1 \mathrm{H}), 4.33(\mathrm{dd}, J=8.0 \mathrm{~Hz}, 6.4 \mathrm{~Hz}, 0.32 \mathrm{H})$, $3.80(\mathrm{~s}, 0.96 \mathrm{H}), 3.71(\mathrm{~s}, 3 \mathrm{H}), 3.59(\mathrm{~s}, 0.96 \mathrm{H}), 3.54$ (s, 3H), 3.12 (dd, $J=13.2 \mathrm{~Hz}, 6.4$ $\mathrm{Hz}, 0.32 \mathrm{H}), 3.01-2.86(\mathrm{~m}, 2.32 \mathrm{H}), 1.92$ (s, 3H), 1.90 (s, 0.96H); ${ }^{13}$ C NMR (100 MHz, MeOD-d 4$): \delta 171.7,169.2,160.0,156.2,131.4,127.8,115.1,107.7,104.2,101.8$, 100.8, 62.8, 62.7, 54.6, 54.2, 51.8, 31.8, 21.12, 21.07; IR (neat, $\mathbf{~ c m}^{-1}$ ): v 3350, 1664, 1611, 1518, 1470, 1161, 1034, 850; MS (m/z, ESI): 283, 241, 236, 208; HRMS calculated for $\mathrm{C}_{13} \mathrm{H}_{19} \mathrm{~N}_{2} \mathrm{O}_{5}(\mathrm{M}+\mathrm{H})^{+}: 283.1288$; Found: $283.129 ; \mathbf{R}_{\mathbf{f}}=0.2$ (EA).

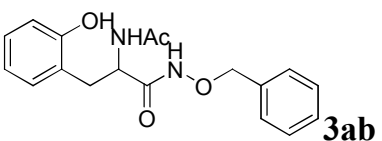

White solid, $85 \%$ yield (56 mg) from $N$-(benzyloxy)acrylamide (35.4 mg, $0.2 \mathrm{mmol}, 1$ equiv); m.p: $165-166{ }^{\circ} \mathrm{C} ;{ }^{1} \mathbf{H}$ NMR (400 MHz, MeOD-d 4 ): $\delta 7.30$ (s, 5H), 7.09-7.05 $(\mathrm{m}, 2 \mathrm{H}), 6.80(\mathrm{~d}, J=8.0 \mathrm{~Hz},, 1 \mathrm{H}), 6.75(\mathrm{t}, J=7.2 \mathrm{~Hz}, 1 \mathrm{H}), 4.73(\mathrm{~d}, J=10.8 \mathrm{~Hz}, 1 \mathrm{H})$, $4.60(\mathrm{~d}, J=11.2 \mathrm{~Hz}, 1 \mathrm{H}), 4.50(\mathrm{t}, J=7.6 \mathrm{~Hz}, 1 \mathrm{H}), 2.98(\mathrm{~d}, J=7.6 \mathrm{~Hz}, 2 \mathrm{H}), 1.90$ (s, $3 \mathrm{H}) ;{ }^{13}$ C NMR (100 MHz, MeOD-d 4 ): $\delta$ 173.0, 170.7, 156.8, 136.6, 132.3, 130.4, $129.6,129.4,129.3,124.2,120.6,116.0,79.1,52.9,33.8,22.4$; IR (neat, $\mathbf{c m}^{-1}$ ): v 3351, 3177, 1658, 1603, 1550, 1480, 1260, 745, 697; MS (m/z, ESI): 329, 206; HRMS calculated for $\mathrm{C}_{18} \mathrm{H}_{21} \mathrm{~N}_{2} \mathrm{O}_{4}(\mathrm{M}+\mathrm{H})^{+}: 329.1496$; Found: 329.1502; $\mathbf{R}_{\mathbf{f}}=0.2$ (EA).

X-ray crystallography of $\mathbf{3 a b}$ : 

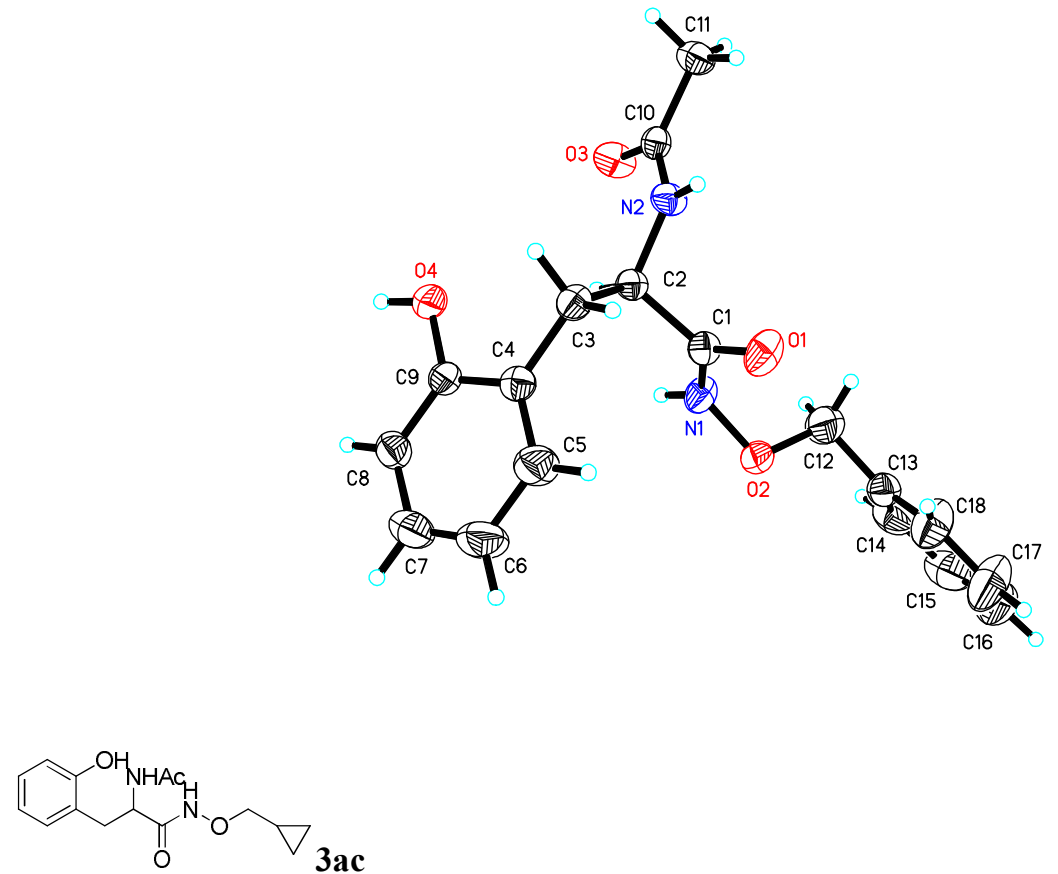

White solid, $89 \%$ yield (52 mg) from $N$-(cyclopropylmethoxy)acrylamide (28.2 $\mathrm{mg}$, 0.2 mmol, 1 equiv); m.p: $169-170{ }^{\circ} \mathrm{C} ;{ }^{1}$ H NMR (400 MHz, MeOD-d 4 ): $\delta$ 7.07-7.03 $(\mathrm{m}, 2 \mathrm{H}), 6.78(\mathrm{~d}, J=8.0 \mathrm{~Hz}, 1 \mathrm{H}), 6.73(\mathrm{t}, J=7.6 \mathrm{~Hz}, 1 \mathrm{H}), 4.51(\mathrm{t}, J=7.2 \mathrm{~Hz}, 1 \mathrm{H})$, $3.52(\mathrm{dd}, J=10.4 \mathrm{~Hz}, 7.2 \mathrm{~Hz}, 1 \mathrm{H}), 3.42(\mathrm{dd}, J=10.4 \mathrm{~Hz}, 7.2 \mathrm{~Hz}, 1 \mathrm{H}), 2.99$ (d, $J=7.2$ $\mathrm{Hz}, 2 \mathrm{H}), 1.92$ (s, 3H), 1.02-0.92 (m, 1H), 0.49 (d, $J=7.6 \mathrm{~Hz}, 2 \mathrm{H}), 0.22-0.14(\mathrm{~m}, 2 \mathrm{H})$; ${ }^{13}$ C NMR (100 MHz, MeOD-d 4 ): $\delta$ 173.0, 170.4, 156.7, 132.3, 129.3, 124.2, 120.5, 115.9, 81.9, 52.9, 33.8, 22.4, 10.0, 3.5, 3.4; IR (neat, cm$^{-1}$ ): $v$ 3241, 1665, 1626, 1556, 1454, 755; MS (m/z, ESI): 293, 206, 178; HRMS calculated for $\mathrm{C}_{15} \mathrm{H}_{21} \mathrm{~N}_{2} \mathrm{O}_{4}(\mathrm{M}+\mathrm{H})^{+}$: 293.1496; Found: 293.1504; $\mathbf{R}_{\mathbf{f}}=0.2$ (EA).

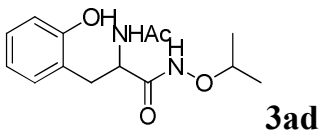

White solid, $75 \%$ yield (42 mg) from $N$-isopropoxyacrylamide $(25.8 \mathrm{mg}, 0.2 \mathrm{mmol}, 1$ equiv); m.p: $174-175{ }^{\circ} \mathrm{C} ;{ }^{1} \mathbf{H}$ NMR (400 MHz, MeOD-d $\left.{ }_{4}\right): \delta$ 7.07-7.03 (m, 2H), 6.78 $(\mathrm{d}, J=7.6 \mathrm{~Hz}, 1 \mathrm{H}), 6.73(\mathrm{t}, J=7.6 \mathrm{~Hz}, 1 \mathrm{H}), 4.52(\mathrm{t}, J=7.6 \mathrm{~Hz}, 1 \mathrm{H}), 3.89$ (heptet, $J=$ $6.0 \mathrm{~Hz}, 1 \mathrm{H}), 2.99$ (d, $J=7.2 \mathrm{~Hz}, 2 \mathrm{H}), 1.92(\mathrm{~s}, 3 \mathrm{H}), 1.10(\mathrm{~d}, J=6.4 \mathrm{~Hz}, 3 \mathrm{H}), 1.02$ (d, $J$ $=6.0 \mathrm{~Hz}, 3 \mathrm{H}) ;{ }^{13} \mathbf{C}$ NMR (100 MHz, MeOD-d 4$): \delta 173.0,170.8,156.7,132.3,129.3$, 124.2, 120.5, 115.9, 78.9, 53.0, 33.8, 22.4, 20.7, 20.69; IR (neat, $\mathbf{~ c m}^{-1}$ ): v 3326, 1658, 1600, 1481, 1021, 752; MS (m/z, ESI): 281, 206, 178; HRMS calculated for $\mathrm{C}_{14} \mathrm{H}_{21} \mathrm{~N}_{2} \mathrm{O} 4(\mathrm{M}+\mathrm{H})^{+}: 281.1496$; Found: $281.1498 ; \mathbf{R}_{\mathbf{f}}=0.1(\mathrm{EA}: \mathrm{PE}=1: 1)$.

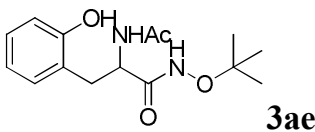

White solid, $66 \%$ yield (39 mg) from $\mathrm{N}$-(tert-butoxy)acrylamide $(28.6 \mathrm{mg}, 0.2 \mathrm{mmol}$, 1 equiv); m.p: $183-184{ }^{\circ} \mathrm{C} ;{ }^{1} \mathbf{H}$ NMR (400 MHz, MeOD-d 4$): \delta$ 7.09-7.03 (m, 2H), 
6.79-6.72 (m, 2H), 4.59 (t, $J=7.6 \mathrm{~Hz}, 1 \mathrm{H}), 2.99(\mathrm{~d}, J=7.6 \mathrm{~Hz}, 2 \mathrm{H}), 1.92(\mathrm{~s}, 3 \mathrm{H})$, 1.12 (s, 9H); ${ }^{13}$ C NMR (100 MHz, MeOD-d 4 ): $\delta$ 171.6, 170.6, 155.4, 130.9, 127.9, 122.9, 119.2, 114.6, 81.9, 51.7, 32.3, 25.2, 21.0; IR (neat, $\mathbf{c m}^{-1}$ ): v 3337, 3212, 1663, 1621, 1552, 1455, 1366, 750; MS (m/z, ESI): 295, 239, 206, 178; HRMS calculated for $\mathrm{C}_{15} \mathrm{H}_{23} \mathrm{~N}_{2} \mathrm{O}_{4}(\mathrm{M}+\mathrm{H})^{+}:$295.1652; Found: 295.1657; $\mathbf{R}_{\mathbf{f}}=0.2(\mathrm{EA}: \mathrm{PE}=1: 1)$.

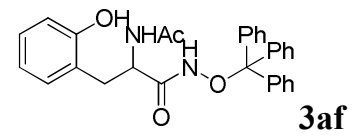

Yellow solid, $15 \%$ yield (36 mg) from $N$-(trityloxy)acrylamide $(164.5 \mathrm{mg}, 0.5 \mathrm{mmol}$, 1 equiv); m.p: $186-187{ }^{\circ} \mathrm{C}$; ${ }^{1}$ H NMR (400 MHz, DMSO-d $)$ ): $\delta 10.36$ (s, 1H), 9.27 (s, $1 \mathrm{H}), 7.85(\mathrm{~d}, J=8.4 \mathrm{~Hz}, 1 \mathrm{H}), 7.31(\mathrm{~s}, 15 \mathrm{H}), 6.96(\mathrm{t}, J=7.6 \mathrm{~Hz}, 1 \mathrm{H}), 6.87(\mathrm{~d}, J=7.2$ $\mathrm{Hz}, 1 \mathrm{H}), 6.71(\mathrm{~d}, J=8.0 \mathrm{~Hz}, 1 \mathrm{H}), 6.57(\mathrm{t}, J=7.6 \mathrm{~Hz}, 1 \mathrm{H}), 4.38-4.33(\mathrm{~m}, 1 \mathrm{H})$, 2.47-2.41 (m, 2H), 1.67 (s, 3H); ${ }^{13}$ C NMR (100 MHz, DMSO-d $\left.)_{6}\right): \delta 170.1,169.4$, $155.7,142.7,130.6,129.4,128.0,127.9,124.2,119.0,115.1,92.3,50.3,31.8,22.8$; IR (neat, $\mathbf{c m}^{-1}$ ): v 3300, 1739, 1668, 755, 701; MS (m/z, ESI): $503(\mathrm{M}+\mathrm{Na})^{+}, 244$, 243; HRMS calculated for $\mathrm{C}_{30} \mathrm{H}_{28} \mathrm{~N}_{2} \mathrm{O}_{4} \mathrm{Na}(\mathrm{M}+\mathrm{Na})^{+}:$503,1941; Found: 503.1941; $\mathbf{R}_{\mathbf{f}}$ $=0.3(\mathrm{EA}: \mathrm{PE}=1: 1)$.

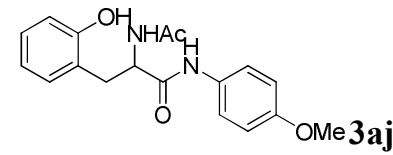

Yellow solid, 10\% yield (16 mg) from $N$-(4-methoxyphenyl)acrylamide (81 mg, 0.5 mmol, 1 equiv); m.p: $210-211{ }^{\circ} \mathrm{C} ;{ }^{1} \mathbf{H}$ NMR (400 MHz, DMSO-d 6 ): $\delta 9.75$ (s, $\left.1 \mathrm{H}\right)$, $9.41(\mathrm{~s}, 1 \mathrm{H}), 8.10(\mathrm{~d}, J=8.0 \mathrm{~Hz}, 1 \mathrm{H}), 7.47-7.45(\mathrm{~m}, 2 \mathrm{H}), 7.07$ (dd, $J=7.6 \mathrm{~Hz}, 1.2 \mathrm{~Hz}$, $1 \mathrm{H}), 7.00(\mathrm{td}, J=7.6 \mathrm{~Hz}, 1.2 \mathrm{~Hz}, 1 \mathrm{H}), 6.87-6.84(\mathrm{~m}, 2 \mathrm{H}), 6.78(\mathrm{~d}, J=8.0 \mathrm{~Hz}, 1 \mathrm{H})$, $6.67(\mathrm{td}, J=7.2 \mathrm{~Hz}, 0.8 \mathrm{~Hz}, 1 \mathrm{H}), 4.63-4.57(\mathrm{~m}, 1 \mathrm{H}), 3.71(\mathrm{~s}, 3 \mathrm{H}), 2.96(\mathrm{dd}, J=14.0$ $\mathrm{Hz}, 6.4 \mathrm{~Hz}, 1 \mathrm{H}), 2.82(\mathrm{dd}, J=14.0 \mathrm{~Hz}, 8.4 \mathrm{~Hz}, 1 \mathrm{H}), 1.80(\mathrm{~s}, 3 \mathrm{H}) ;{ }^{13} \mathbf{C}$ NMR (100 MHz, DMSO-d $\left.\mathbf{d}_{\mathbf{6}}\right): \delta 169.6,168.7,154.9,154.8,131.5,129.8,127.0,123.3,120.6$, 118.2, 114.3, 113.2, 54.6, 53.0, 31.6, 22.0; IR (neat, $\mathbf{~ c m}^{-1}$ ): $v 3347,3253,1659,1620$, 1600, 1549, 1510, 1455, 842, 751; MS (m/z, ESI): 329, 327, 206; HRMS calculated for $\mathrm{C}_{18} \mathrm{H}_{21} \mathrm{~N}_{2} \mathrm{O}_{4}(\mathrm{M}+\mathrm{H})^{+}: 329.1496$; Found: $329.1492 ; \mathbf{R}_{\mathbf{f}}=0.1(\mathrm{EA}: \mathrm{PE}=1: 1)$.

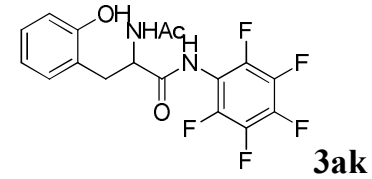

White solid, $18 \%$ yield $(35 \mathrm{mg}$ ) from $N$-(perfluorophenyl)acrylamide $(111 \mathrm{mg}, 0.5$ mmol, 1 equiv); m.p: $234-235{ }^{\circ} \mathrm{C} ;{ }^{1} \mathbf{H}$ NMR (400 MHz, DMSO-d $\left.{ }_{6}\right): \delta 10.08$ (s, $\left.1 \mathrm{H}\right)$, $9.49(\mathrm{~s}, 1 \mathrm{H}), 8.24(\mathrm{~d}, J=8.0 \mathrm{~Hz}, 1 \mathrm{H}), 7.10(\mathrm{~d}, J=7.2 \mathrm{~Hz}, 1 \mathrm{H}), 7.04(\mathrm{td}, J=7.6 \mathrm{~Hz}$, $1.6 \mathrm{~Hz}, 1 \mathrm{H}), 6.80(\mathrm{~d}, J=7.6 \mathrm{~Hz}, 1 \mathrm{H}), 6.70(\mathrm{t}, J=7.6 \mathrm{~Hz}, 1 \mathrm{H}), 4.76(\mathrm{dd}, J=13.6 \mathrm{~Hz}$, $8.8 \mathrm{~Hz}, 1 \mathrm{H}), 3.06$ (dd, $J=14.0 \mathrm{~Hz}, 5.2 \mathrm{~Hz}, 1 \mathrm{H}), 2.84$ (dd, $J=14.0 \mathrm{~Hz}, 9.2 \mathrm{~Hz}, 1 \mathrm{H})$, 1.81 (s, 3H); ${ }^{13}$ C NMR (100 MHz, DMSO-d 6 ): $\delta$ 171.7, 169.9, 155.9, $142.8\left(\mathrm{dd}, J_{\mathrm{C}-\mathrm{F}}\right.$ $=246.6,6.1 \mathrm{~Hz}), 139.1\left(\mathrm{dt}, J_{\mathrm{C}-\mathrm{F}}=248.1,13.4 \mathrm{~Hz}\right), 137.3\left(\mathrm{dt}, J_{\mathrm{C}-\mathrm{F}}=248.2,13.4 \mathrm{~Hz}\right)$, $130.5,137.7,123.5,118.8,114.8,112.9(\mathrm{t}, J=15.3 \mathrm{~Hz}), 52.7,32.1,22.5 ;{ }^{19} \mathrm{~F}$ NMR (376M, DMSO-d $)) \delta-140.2,153.3(\mathrm{q}, J=16 \mathrm{~Hz}),-159.0$; IR (neat, $\mathbf{c m}^{-1}$ ): $v$ 3328, 3217, 2926, 2854, 1682, 1601, 1551, 1501, 1457, 1221, 756; MS (m/z, ESI): 387, 
342, 232; HRMS calculated for $\mathrm{C}_{18} \mathrm{H}_{13} \mathrm{~F}_{5} \mathrm{~N}_{2} \mathrm{O}_{3}(\mathrm{M}+\mathrm{H})^{+}$: 389.0919; Found: 389.0919; $\mathbf{R}_{\mathbf{f}}=0.3(\mathrm{EA}: \mathrm{PE}=1: 1)$.

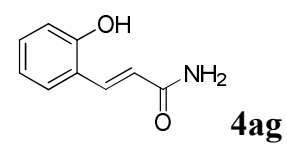

White solid, $55 \%$ yield (18 mg) from acrylamide (14.2 mg, $0.2 \mathrm{mmol}, 1$ equiv); m.p: 196-197 ${ }^{\circ} \mathrm{C} ;{ }^{1}$ H NMR (400 MHz, MeOD-d 4$): \delta 7.86$ (d, $\left.J=16.0 \mathrm{~Hz}, 1 \mathrm{H}\right), 7.46(\mathrm{~d}, J$ $=7.6 \mathrm{~Hz}, 1 \mathrm{H}), 7.19-7.15(\mathrm{~m}, 1 \mathrm{H}), 6.84-6.80(\mathrm{~m}, 2 \mathrm{H}), 6.74(\mathrm{~d}, J=16.0 \mathrm{~Hz}, 1 \mathrm{H}) ;{ }^{13} \mathrm{C}$ NMR (100 MHz, MeOD-d 4 ): $\delta$ 167.8, 153.9, 134.8, 127.9, 125.7, 118.9, 116.8, 116.6, 112.8; IR (neat, $\mathbf{c m}^{-1}$ ): $v$ 3425, 3176, 1646, 1577, 1489, 1457, 949, 745; MS (m/z, ESI): 164, 121; HRMS calculated for $\mathrm{C}_{9} \mathrm{H}_{10} \mathrm{NO}_{2}(\mathrm{M}+\mathrm{H})^{+}$: 164.0706; Found: $164.0710 ; \mathbf{R}_{\mathbf{f}}=0.2(\mathrm{EA})$.

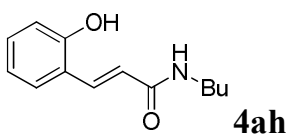

White solid, 53\% yield (23 mg) from $N$-butylacrylamide (25.4 mg, $0.2 \mathrm{mmol}, 1$ equiv); m.p: $139-140{ }^{\circ} \mathrm{C} ;{ }^{1} \mathbf{H}$ NMR (400 MHz, MeOD-d 4$): \delta 7.82$ (d, $\left.J=15.6 \mathrm{~Hz}, 1 \mathrm{H}\right), 7.44$ $(\mathrm{dd}, J=8.0 \mathrm{~Hz}, 2.0 \mathrm{~Hz}, 1 \mathrm{H}), 7.15$ (td, $J=7.6 \mathrm{~Hz}, 1.2 \mathrm{~Hz}, 1 \mathrm{H}), 6.83-6.80(\mathrm{~m}, 2 \mathrm{H})$, $6.71(\mathrm{~d}, J=15.6 \mathrm{~Hz}, 1 \mathrm{H}), 3.29$ (t, $J=7.2 \mathrm{~Hz}, 2 \mathrm{H}), 1.54$ (quint, $J=7.2 \mathrm{~Hz}, 2 \mathrm{H}$ ), 1.39 (sext, $J=7.2 \mathrm{~Hz}, 2 \mathrm{H}), 0.95(\mathrm{t}, J=7.2 \mathrm{~Hz}, 3 \mathrm{H}) ;{ }^{13} \mathbf{C}$ NMR (100 MHz, MeOD-d 4 ): $\delta$ $169.5,157.9,137.7,131.7,129.7,123.2,121.6,120.6,116.9,40.3,32.6,21.2,14.1$; IR (neat, $\left.\mathbf{c m}^{-1}\right): v$ 3314, 2960, 2929, 1646, 1578, 1551, 1451, 785, 784; MS (m/z, ESI): 220; HRMS calculated for $\mathrm{C}_{13} \mathrm{H}_{18} \mathrm{NO}_{2}(\mathrm{M}+\mathrm{H})^{+}: 220.1332$; Found: 220.1337 ; $\mathbf{R}_{\mathbf{f}}$ $=0.2(\mathrm{EA}: \mathrm{PE}=1: 1)$.

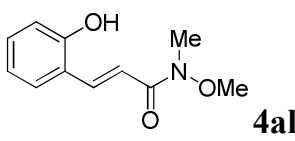

White solid, $42 \%$ yield $(17 \mathrm{mg})$ from $N$-methoxy- $N$-methylacrylamide $(23.0 \mathrm{mg}, 0.2$ mmol, 1 equiv); m.p: $132-133{ }^{\circ} \mathrm{C} ;{ }^{1} \mathbf{H}$ NMR (400 $\left.\mathbf{M H z}, \mathbf{C D C l}_{3}\right): \delta 8.68$ (s, 1H), 8.33 $(\mathrm{d}, J=16.0 \mathrm{~Hz}, 1 \mathrm{H}), 7.53(\mathrm{~d}, J=7.2 \mathrm{~Hz}, 1 \mathrm{H}), 7.20(\mathrm{t}, J=7.6 \mathrm{~Hz}, 1 \mathrm{H}), 7.07$ (d, $J=$ $16.0 \mathrm{~Hz}, 1 \mathrm{H}), 6.94(\mathrm{~d}, J=8.0 \mathrm{~Hz}, 1 \mathrm{H}), 6.86(\mathrm{t}, J=7.6 \mathrm{~Hz}, 1 \mathrm{H}), 3.75(\mathrm{~s}, 3 \mathrm{H}), 3.34$ (s, $3 \mathrm{H}) ;{ }^{13}$ C NMR (100 MHz, MeOD-d 4 ): $\delta$ 169.5, 158.3, 140.9, 132.3, 130.1, 123.2, 120.7, 117.0, 116.2, 62.4, 32.8; IR (neat, $\mathbf{c m}^{-1}$ ): v 3358, 1634, 1581, 1453, 1259, 994, 752; MS (m/z, EI): 207, 147, 118, 103, 89, 63; HRMS calculated for $\mathrm{C}_{11} \mathrm{H}_{13} \mathrm{NO}_{3}$ $(\mathrm{M})^{+}:$207.0895; Found: 207.0893; $\mathbf{R}_{\mathbf{f}}=0.2(\mathrm{DCM}: \mathrm{MeOH}=40: 1)$.

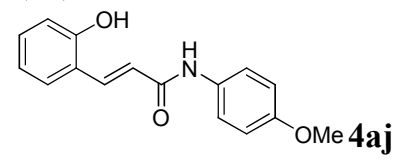

White solid, 39\% yield (21 mg) from $N$-(4-methoxyphenyl)acrylamide (32.4 mg, 0.2 mmol, 1 equiv); m.p: $187-188{ }^{\circ} \mathrm{C} ;{ }^{1} \mathbf{H}$ NMR (400 MHz, MeOD-d 4 ): $\delta 7.93$ (d, $J=$ $16.0 \mathrm{~Hz}, 1 \mathrm{H}), 7.57-7.55(\mathrm{~m}, 2 \mathrm{H}), 7.48(\mathrm{~d}, J=8.0 \mathrm{~Hz}, 1 \mathrm{H}), 7.18$ (td, $J=7.6 \mathrm{~Hz}, 1.6 \mathrm{~Hz}$, 1H), 6.92-6.82(m, 5H), 3.77 (s, 3H); ${ }^{13}$ C NMR (100 MHz, MeOD-d 4 ): $\delta$ 167.4, 158.1, $157.8,138.6,133.1,132.0,130.0,123.1,122.9,121.9,120.7,116.9,114.9,55.8$; IR (neat, $\mathbf{c m}^{-1}$ ): $v$ 3254, 1646, 1594, 1509, 1451, 1407, 1235, 828, 748; MS (m/z, ESI): 
270; HRMS calculated for $\mathrm{C}_{16} \mathrm{H}_{16} \mathrm{NO}_{3}(\mathrm{M}+\mathrm{H})^{+}: 270.1125$; Found: 270.1123; $\mathbf{R}_{\mathbf{f}}=0.5$ $(\mathrm{PE}: \mathrm{EA}=1: 1)$.

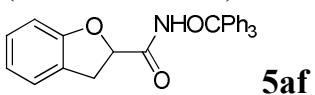

White solid, 30\% yield (25 mg) from $N$-(trityloxy)acrylamide $(65.8 \mathrm{mg}, 0.2 \mathrm{mmol}, 1$ equiv); m.p: $162-163{ }^{\circ} \mathrm{C} ;{ }^{1} \mathbf{H}$ NMR (400 MHz, DMSO-d $\left.{ }_{6}\right): \delta 8.57$ (s, 1H), 7.36 (s, $6 \mathrm{H}), 7.11(\mathrm{~s}, 9 \mathrm{H}), 7.11-7.05(\mathrm{~m}, 2 \mathrm{H}), 6.88(\mathrm{t}, J=7.2 \mathrm{~Hz}, 1 \mathrm{H}), 6.57$ (d, $J=7.6 \mathrm{~Hz}, 1 \mathrm{H})$, $4.96(\mathrm{dd}, J=10.4 \mathrm{~Hz}, 5.6 \mathrm{~Hz}, 1 \mathrm{H}), 3.38(\mathrm{dd}, J=16.0 \mathrm{~Hz}, 11.2 \mathrm{~Hz}, 1 \mathrm{H}), 3.06(\mathrm{dd}, J=$ $16.4 \mathrm{~Hz}, 5.2 \mathrm{~Hz}, 1 \mathrm{H}) ;{ }^{13} \mathbf{C}$ NMR (100 MHz, DMSO-d $)$ ): $\delta 167.9,158.7,142.0,128.9$, $128.7,127.8,127.6,126.6,125.7,124.7,120.6,108.9,92.4,77.9,32.7$; IR (neat, $\left.\mathbf{c m}^{-1}\right):$ v 3196, 1677, 1597, 1525, 1479, 1220, 748; MS (m/z, ESI): $444(\mathrm{M}+\mathrm{Na})^{+}, 244$, 243; HRMS calculated for $\mathrm{C}_{28} \mathrm{H}_{23} \mathrm{NO}_{3} \mathrm{Na}(\mathrm{M}+\mathrm{Na})^{+}: 444.1570$; Found: 444.1574; $\mathbf{R}_{\mathbf{f}}=$ 0.2 (DCM:EA = 8:1).

\section{Deuterium-labeling experiments}

\section{Ortho Deuteration Experiments}

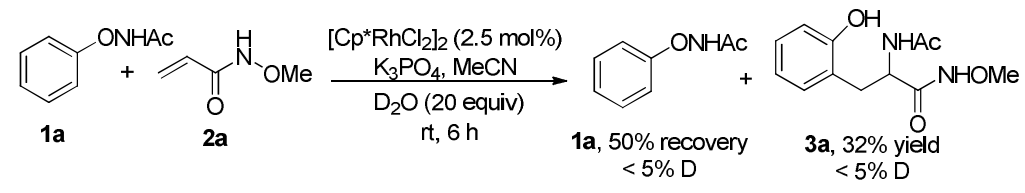

$N$-Phenoxyacetamide (1a, 36.2mg, $0.24 \mathrm{mmol}, 1$ equiv), $N$-methoxyacrylamide (2a, $20.2 \mathrm{mg}, 0.2 \mathrm{mmol}, 1$ equiv), $\left[\mathrm{Cp} * \mathrm{RhCl}_{2}\right]_{2}$ (1.6 mg, $2.5 \mathrm{~mol} \%$ ), and $\mathrm{K}_{3} \mathrm{PO}_{4}(21.2$ $\mathrm{mg}, 0.1 \mathrm{mmol}, 0.5$ equiv) were weighted in a $10 \mathrm{~mL}$ Schlenk tube equipped with a stir bar. The reaction tube was evacuated and refilled with nitrogen three times and then $\mathrm{D}_{2} \mathrm{O}$ (40 mg, $4 \mathrm{mmol}, 20$ equiv) and $\mathrm{CH}_{3} \mathrm{CN}(0.5 \mathrm{~mL})$ were then added. The reaction was stirred at room temperature for $6 \mathrm{~h}$. Afterwards, the reaction mixture was diluted with $\mathrm{MeOH}$ and transferred in a round bottom flask. Silica was added to the flask and volatiles were evaporated under reduced pressure. After purification by flash column chromatography 1a (18 mg, 50\% recovery) and 3a (16 mg, 32\% yield) were isolated and no deuterium incorporation was found in these two compounds. These results imply that the $\mathrm{C}-\mathrm{H}$ activation might be irreversible under the reaction conditions. 


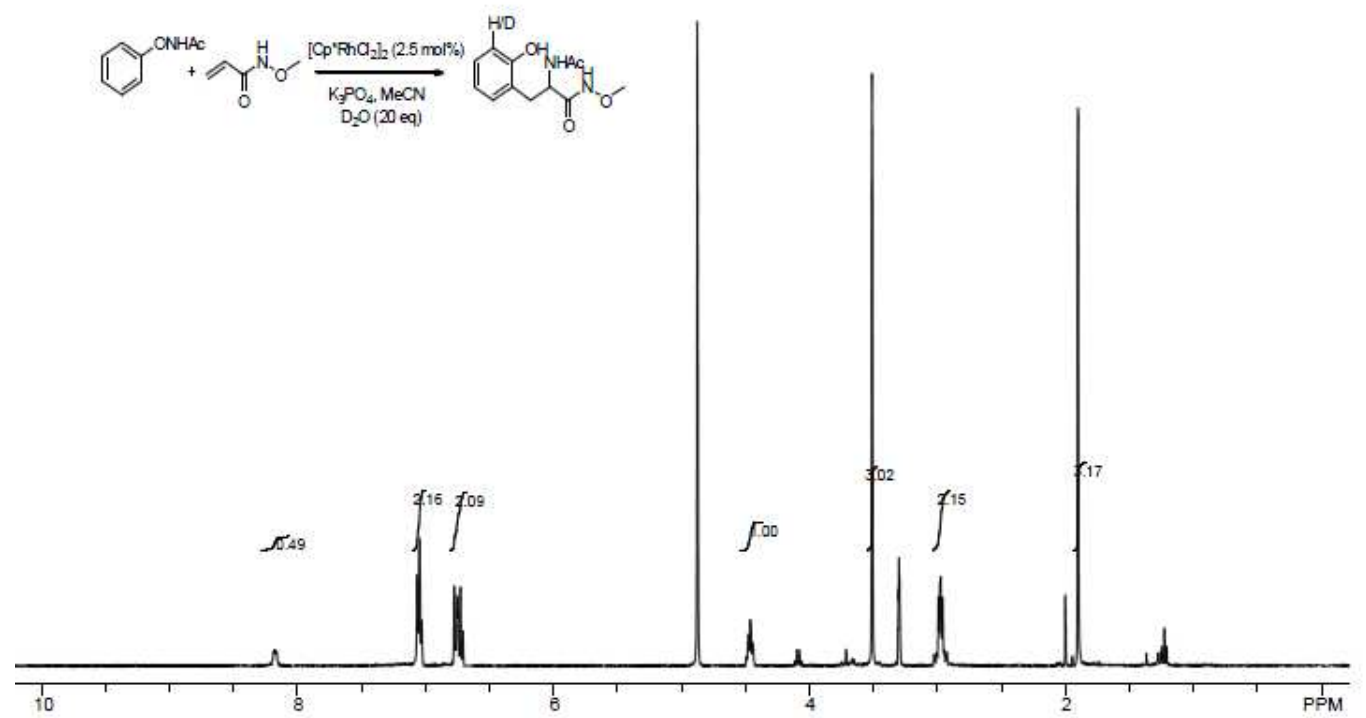

\section{KIE experiments}

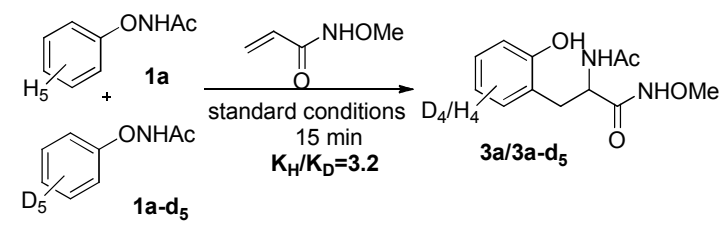

$N$-phenoxyacetamide 1 a (18.2 mg, 0.12 mmol, 1.2 equiv), 1a-d $\mathbf{5}(18.9 \mathrm{mg}, 0.12$ mmol, 1.2 equiv.), $N$-methoxyacrylamide (2a, $10.1 \mathrm{mg}, 0.1 \mathrm{mmol}, 1$ equiv ), $\left[\mathrm{Cp}^{*} \mathrm{RhCl}_{2}\right]_{2}(1.6 \mathrm{mg}, 2.5 \mathrm{~mol} \%), \mathrm{K}_{3} \mathrm{PO}_{4}(10.6 \mathrm{mg}, 0.05 \mathrm{mmol}, 0.5$ equiv. $)$ and $4 \AA$ MS (50 mg)were weighted in a $10 \mathrm{~mL}$ Schlenk tube equipped with a stir bar. The reaction tube was evacuated and refilled with nitrogen three times and then $\mathrm{CH}_{3} \mathrm{CN}$ $(0.25 \mathrm{~mL})$ was added. The reaction was stirred at room temperature for $15 \mathrm{~min}$. Afterwards, the reaction mixture was filtered through a pad of Celite and the filter cake was washed with $\mathrm{MeOH}$. Silica was added to the flask and volatiles were evaporated under reduced pressure. The purification was performed by flash column chromatography on silica gel. The KIE was measured to be 3.2, indicating that the $\mathrm{C}-\mathrm{H}$ bond cleavage process might be involved in the rate-determining step. 


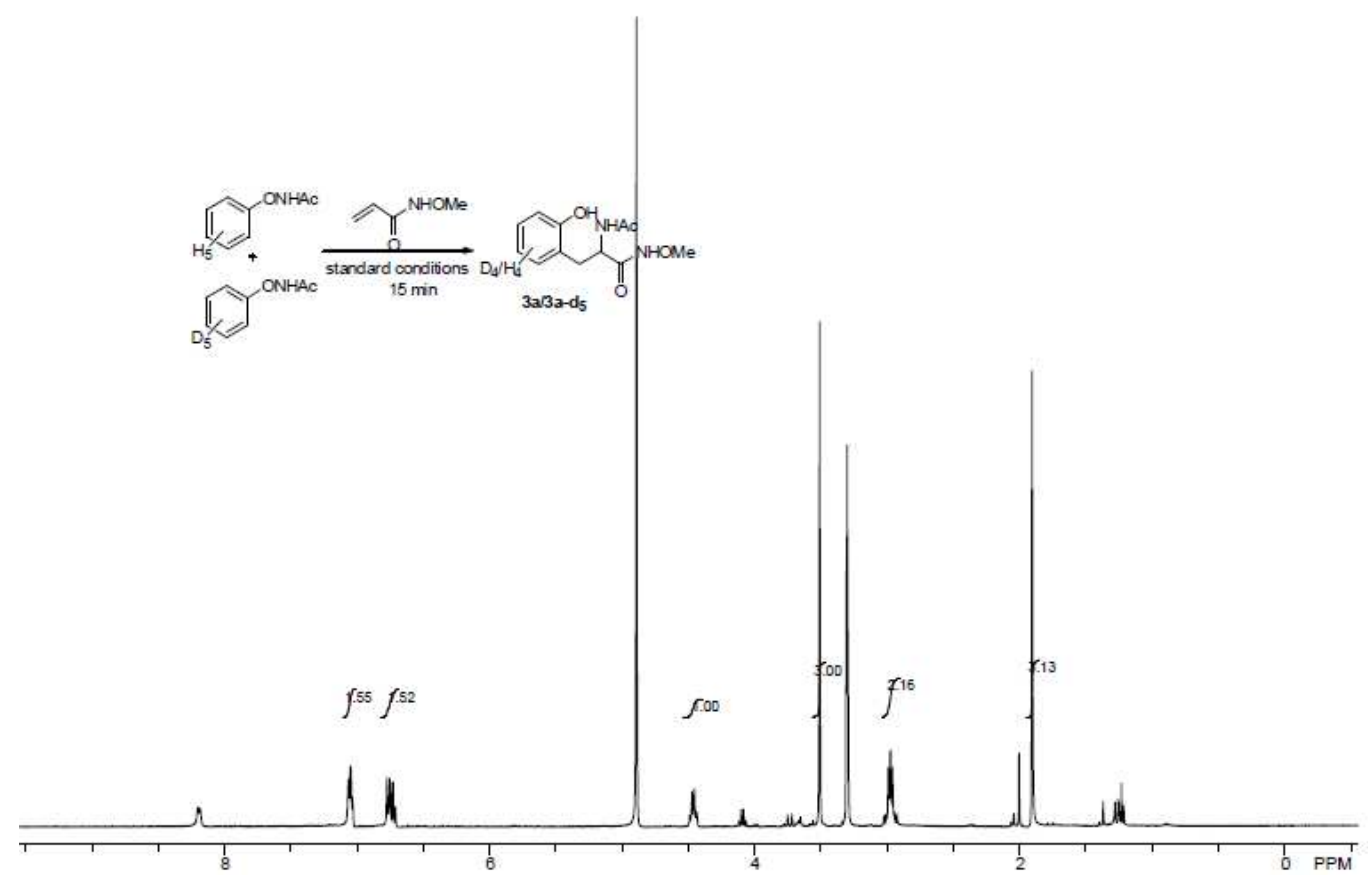

\section{References}

[1] Petrassi, H. M.; Sharpless, K. B.; Kelly, J. W. Org. Lett. 2001, 3, 139.

[2] Liu, G.; Shen, Y.; Zhou, Z.; Lu, X. Angew. Chem., Int. Ed. 2013, 52, 6033.

[3] Wang, F.; Yang, H.; Fu, H.; Pei. Z. Chem. Commun. 2013, 49, 517.

[4] Quan, Z.-J.; Xia, H.-D.; Zhang, Z.; Da, Y.-X.; Wang, X.-C. Tetrahedron 2013, 69, 8368.

[5] Boufroura, H.; Mauduit, M.; Drege, E.; Joseph, D. J. Org.Chem. 2013 , 78, 2346.

[6] Talbot, A. E. P.; Fernandes, A. T.; McKenna, M. J.; Toste, F. D. J. Am. Chem. Soc. 2014, 136, 4101.

[7] Xie, W.; Yang, J.; Wang, B.; Li, B. J. Org. Chem. 2014, 79, 8278

[8] Lebrun, S.; Couture, A.; Deniau, E.; Grandclaudon, P. Synthesis 2006, 20, 3490

[9] Fang, T.; Tan, Q.; Ding, Z.; Liu, B.; Xu, B. Org. Lett. 2014, 16, 2342

[10] Eriksson, J.; Aberg, O.; Langstroem, B. Eur. J . Org. Chem. 2007 , 3, 455

[11] Kumar, D. S.; Panda, G. Tetrahedron 2008, 64, 4162.

[12] Ng, D. Y. W.; Arzt, M.; Wu, Y.; Kuan, L. S.; Lamla, M.; Weil, T. Angew. Chem., Int. Ed. 2014, 53, 324.

[13] Barrett, D. S.; Bridges, J. A.; Dudley, D. T.; Saltiel, A. R.; Fergus, J. H.; Flamme, C. M.; Delaney, A. M.; Kaufman, M.; LePage, S.; Leopold, W. R.; Przybranowski, S. A.; Sebolt-Leopold, J.; Becelaere, Van, K.; Doherty, A. M.; Kennedy, R. M.; Marston, D.; Jr Howard, W. A.; Smith, Y.; Warmus, J. S.; Tecle, H. Bioorg. Med. Chem. Lett. 2008, 18,6501 .

[14] Ren, Z.; Mo, F.; Dong, G. J. Am. Chem. Soc. 2012, 134, 16991.

[15] Weisman, A.; Chen, Y. A.; Hoshino, Y.; Zhang, H.; Shea, K. Biomacromolecules 2014, 15, 3290 . 
6. Copies of ${ }^{1} \mathrm{H},{ }^{13} \mathrm{C},{ }^{19} \mathrm{~F}$ NMR spectra
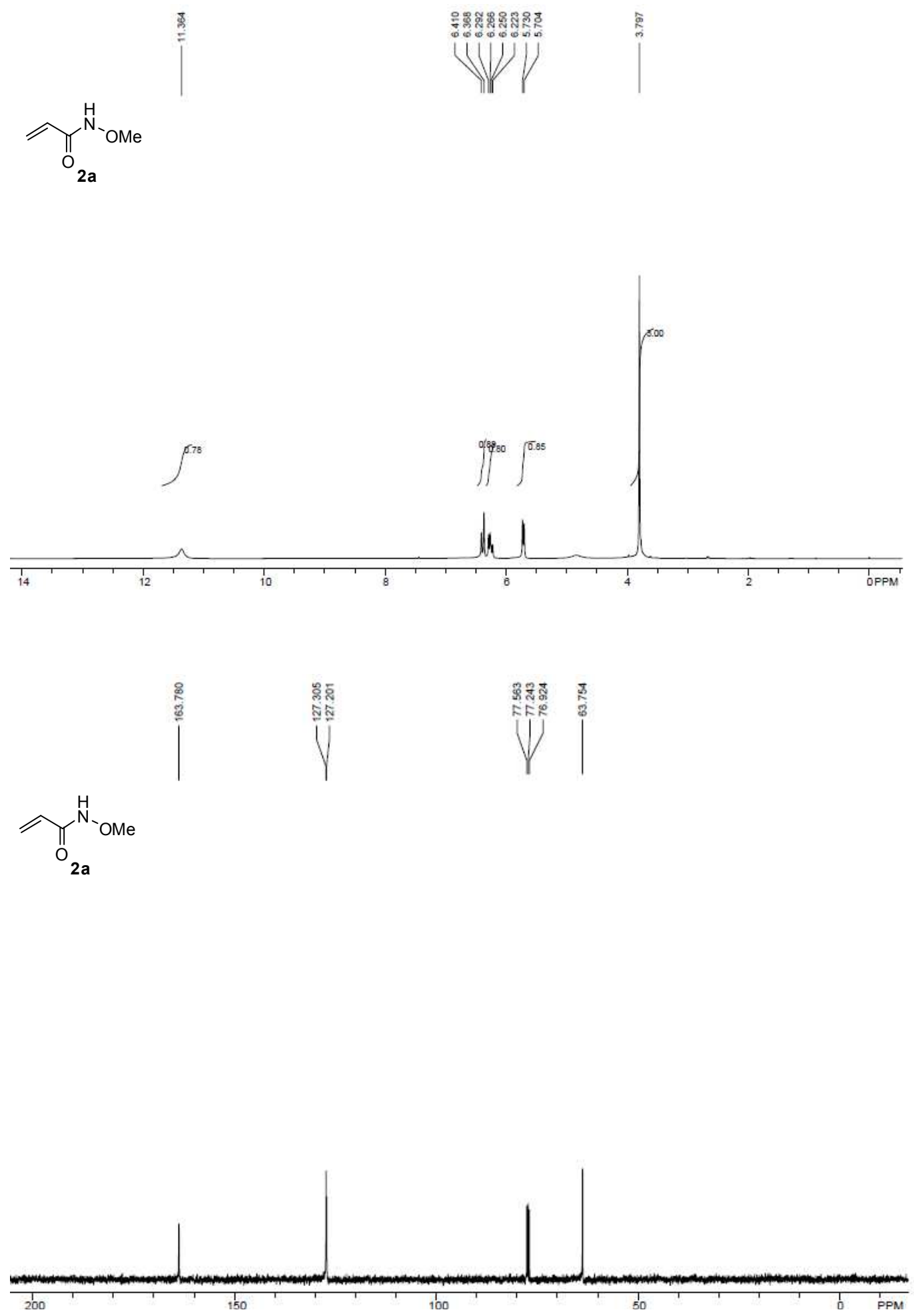


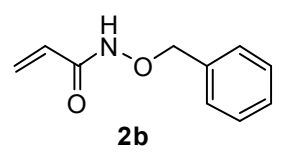

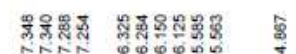

Uij Vij i
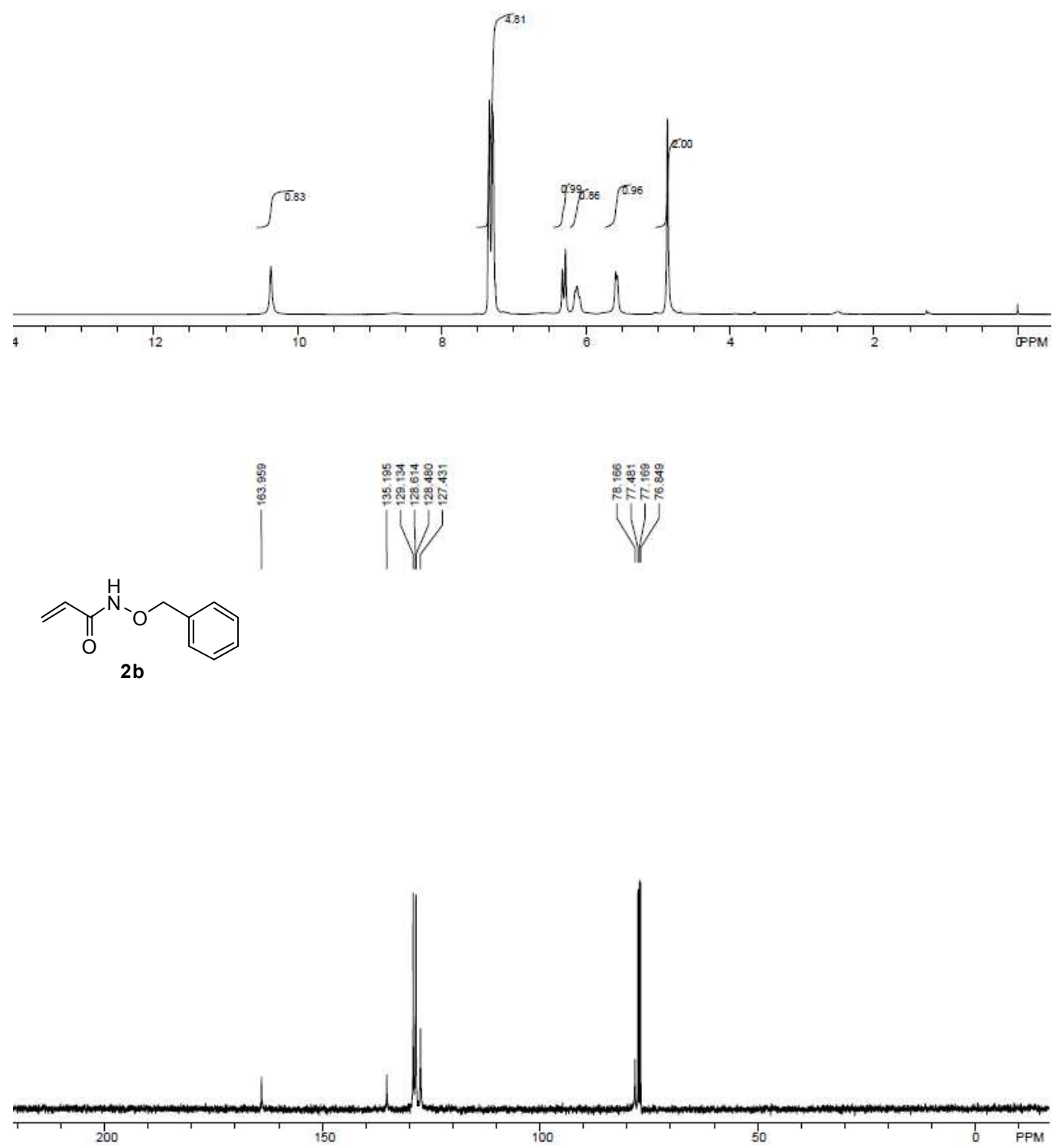

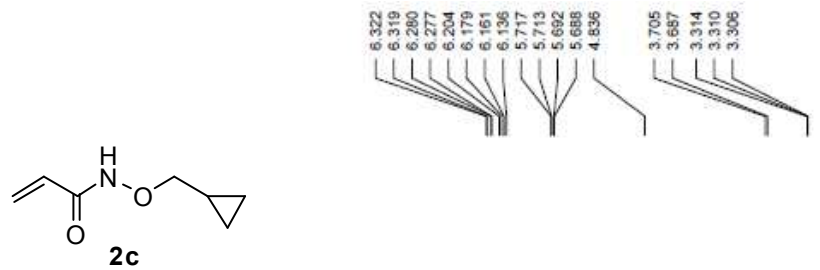

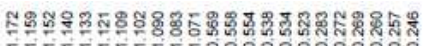

UMUUNJ)JUd」d
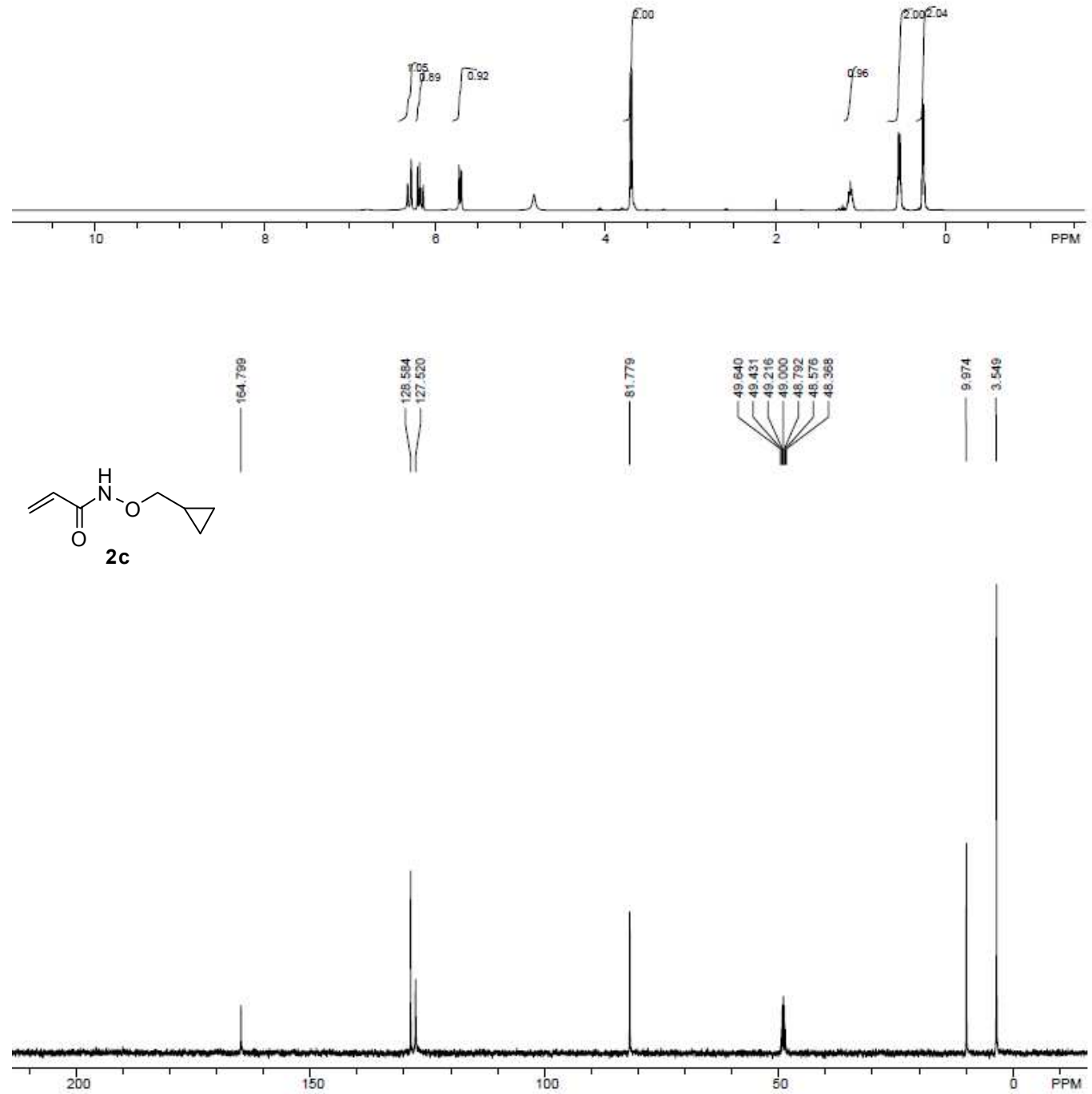

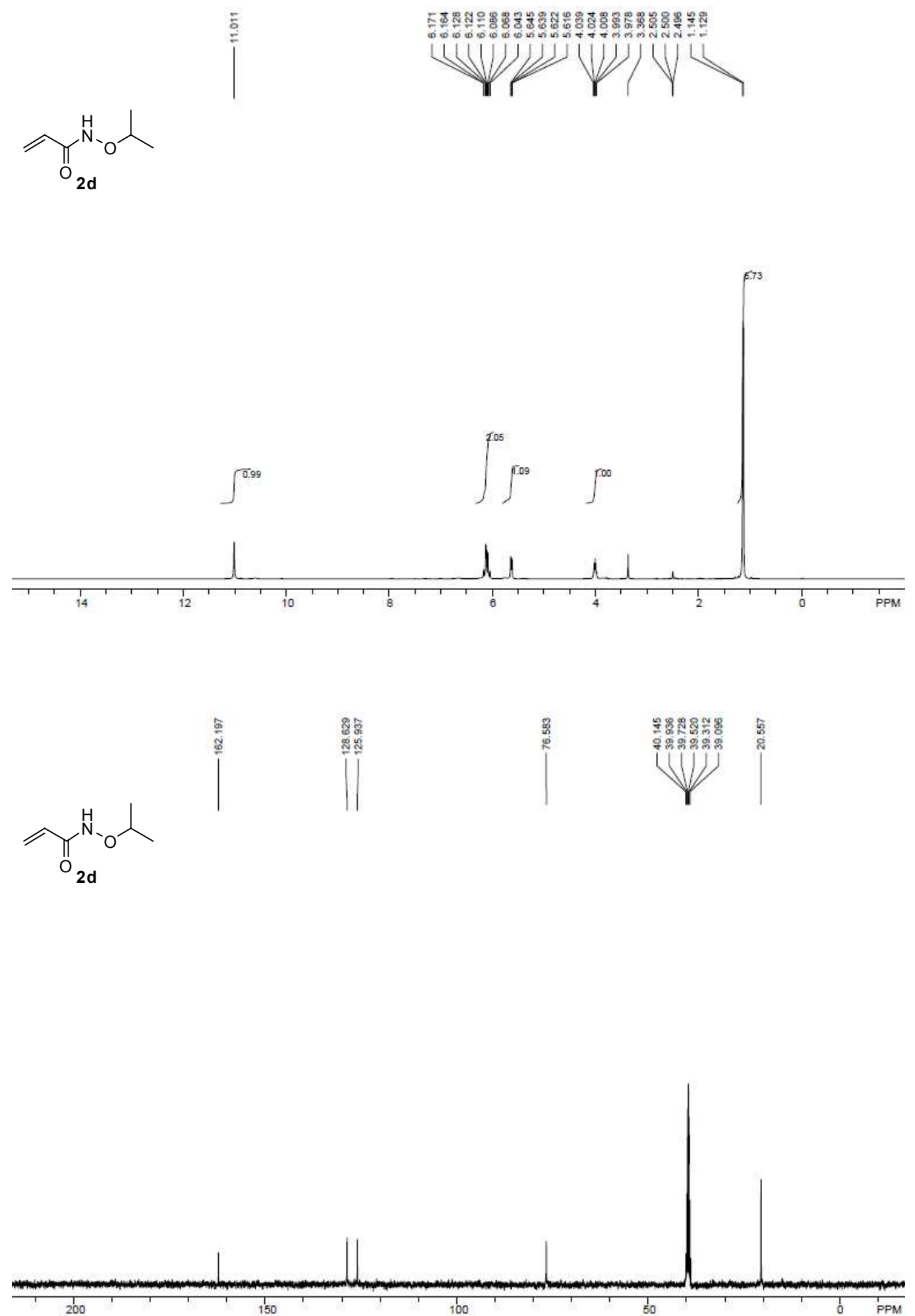


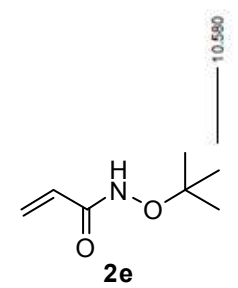

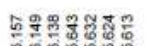

UV

Y

2 e
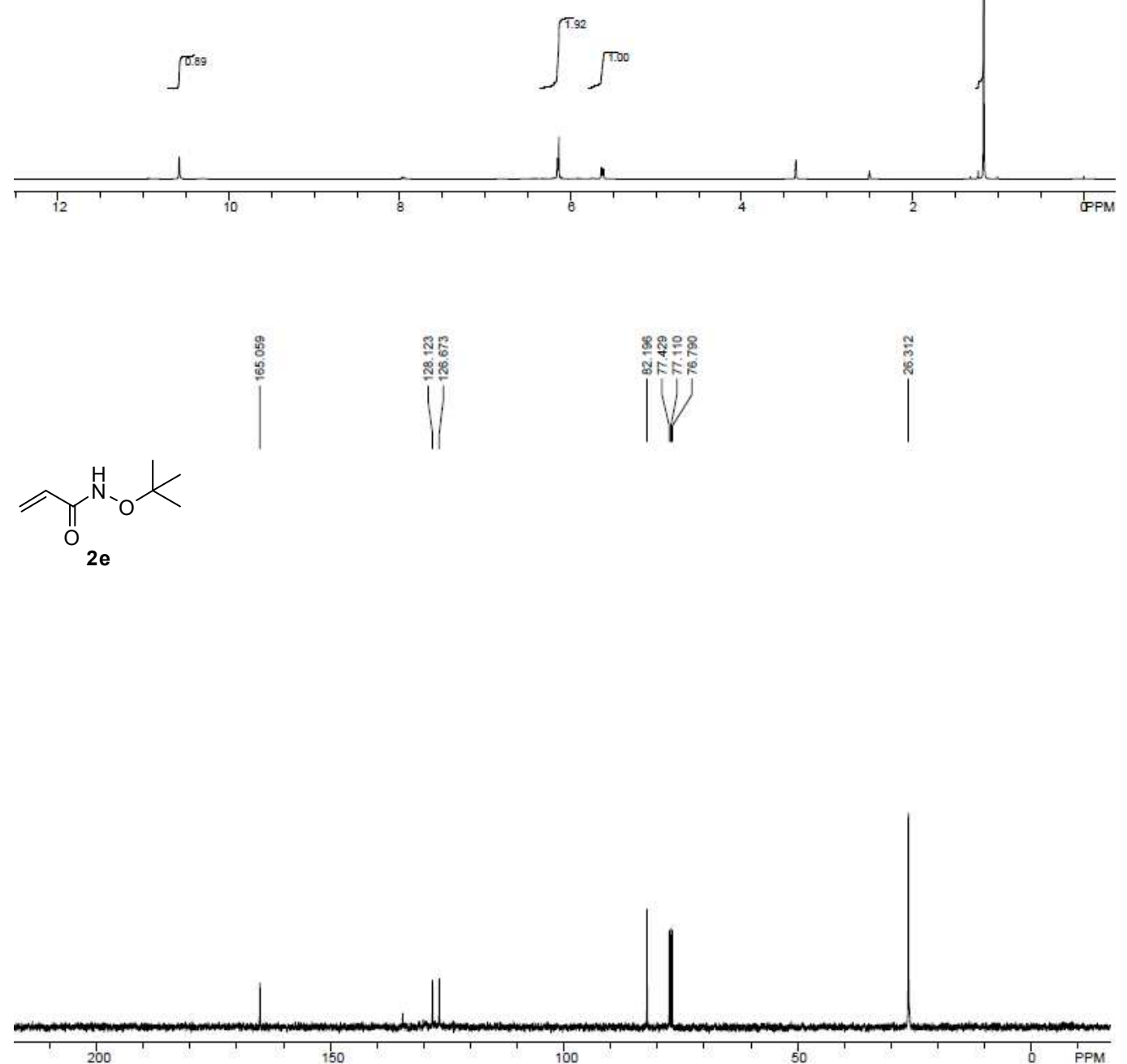

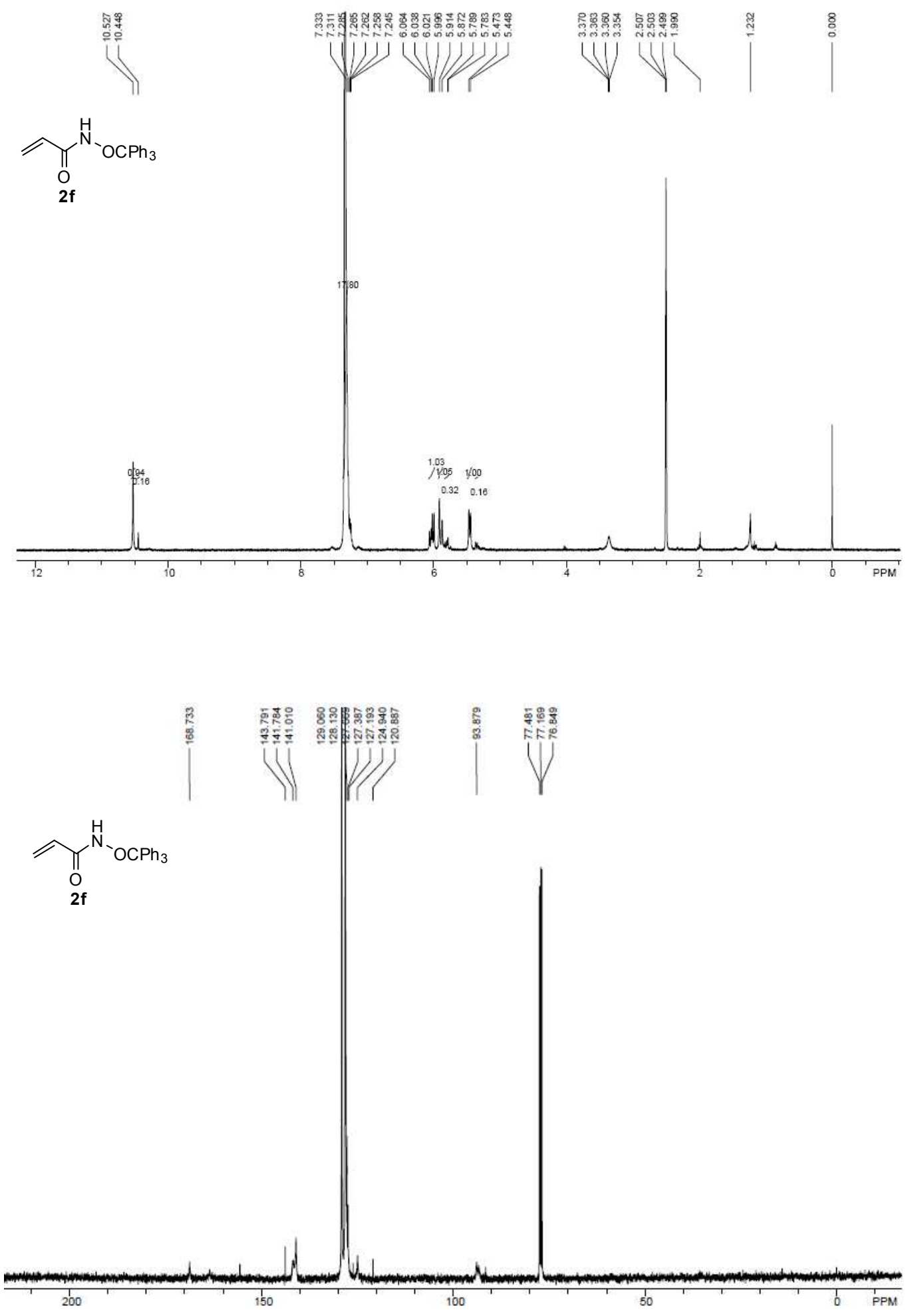

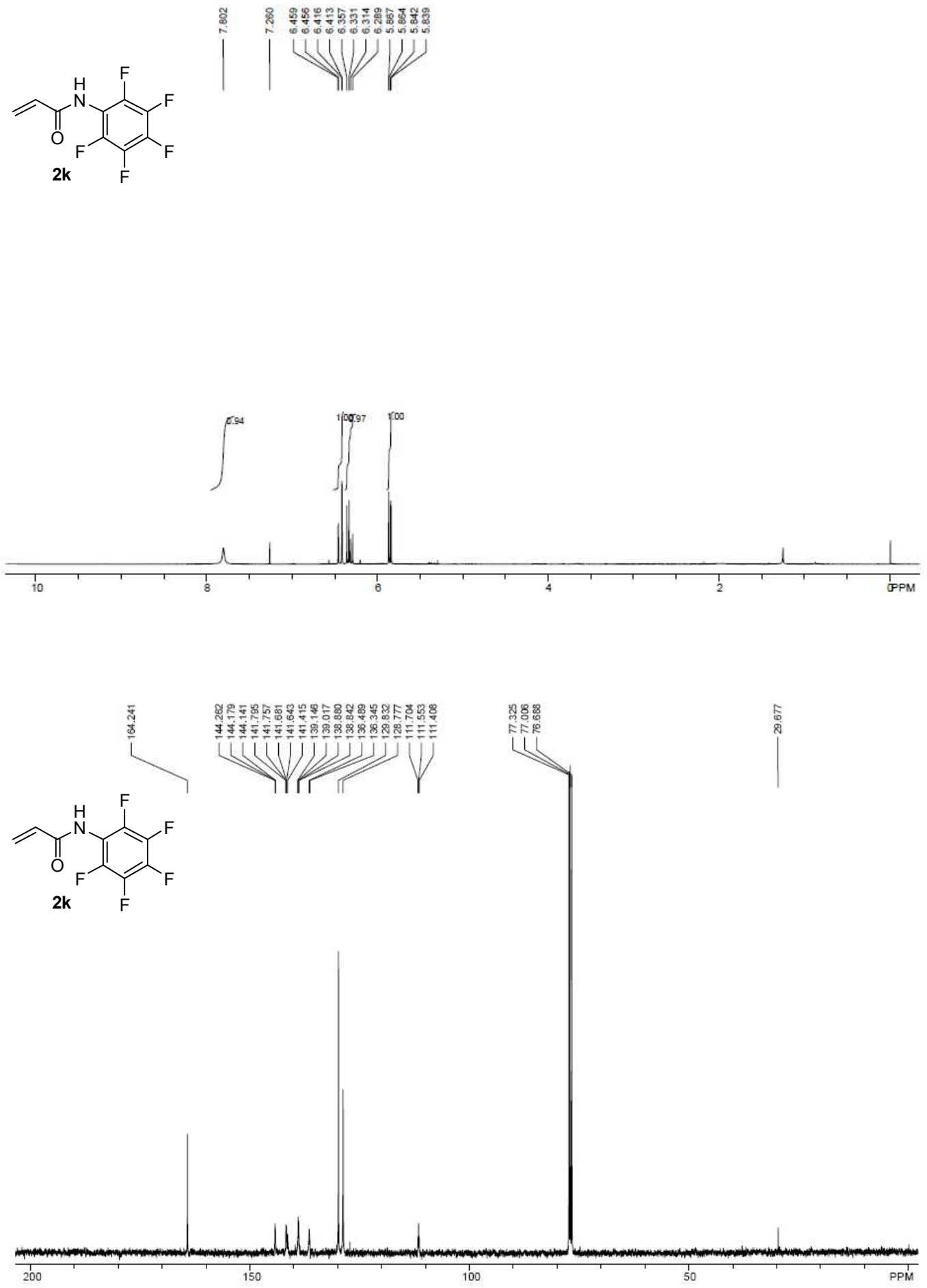

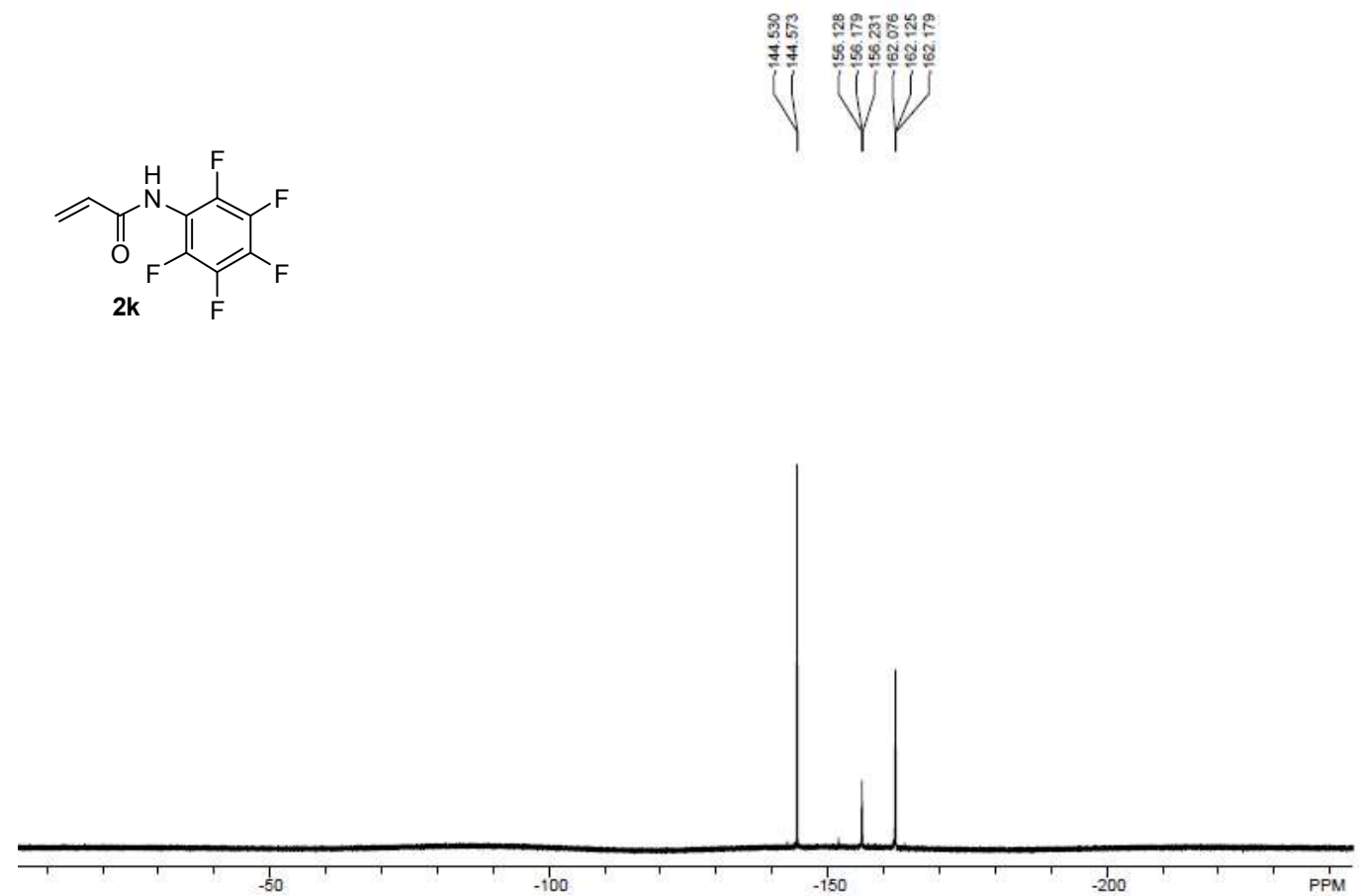


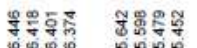

(I) $(1)$
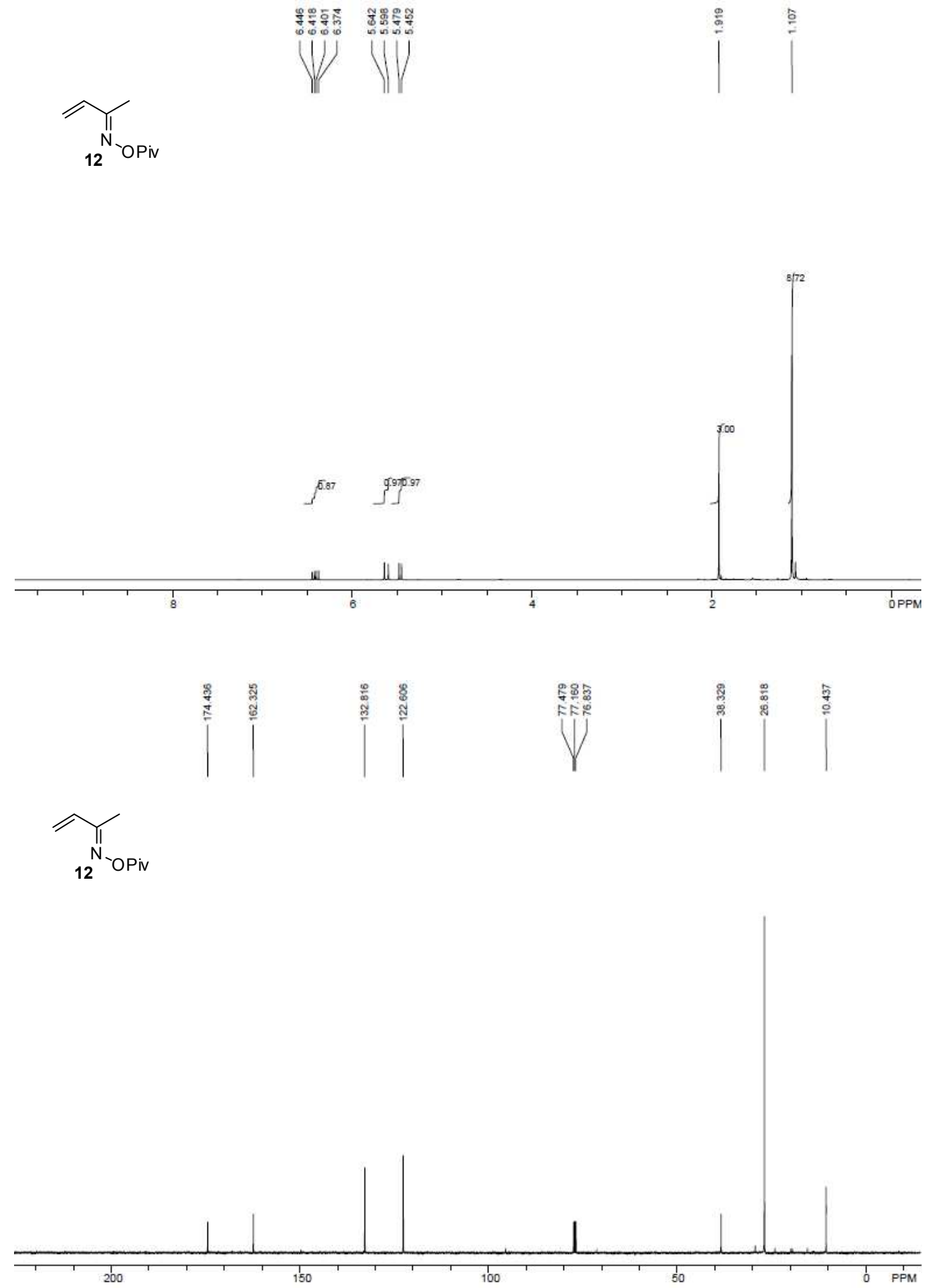

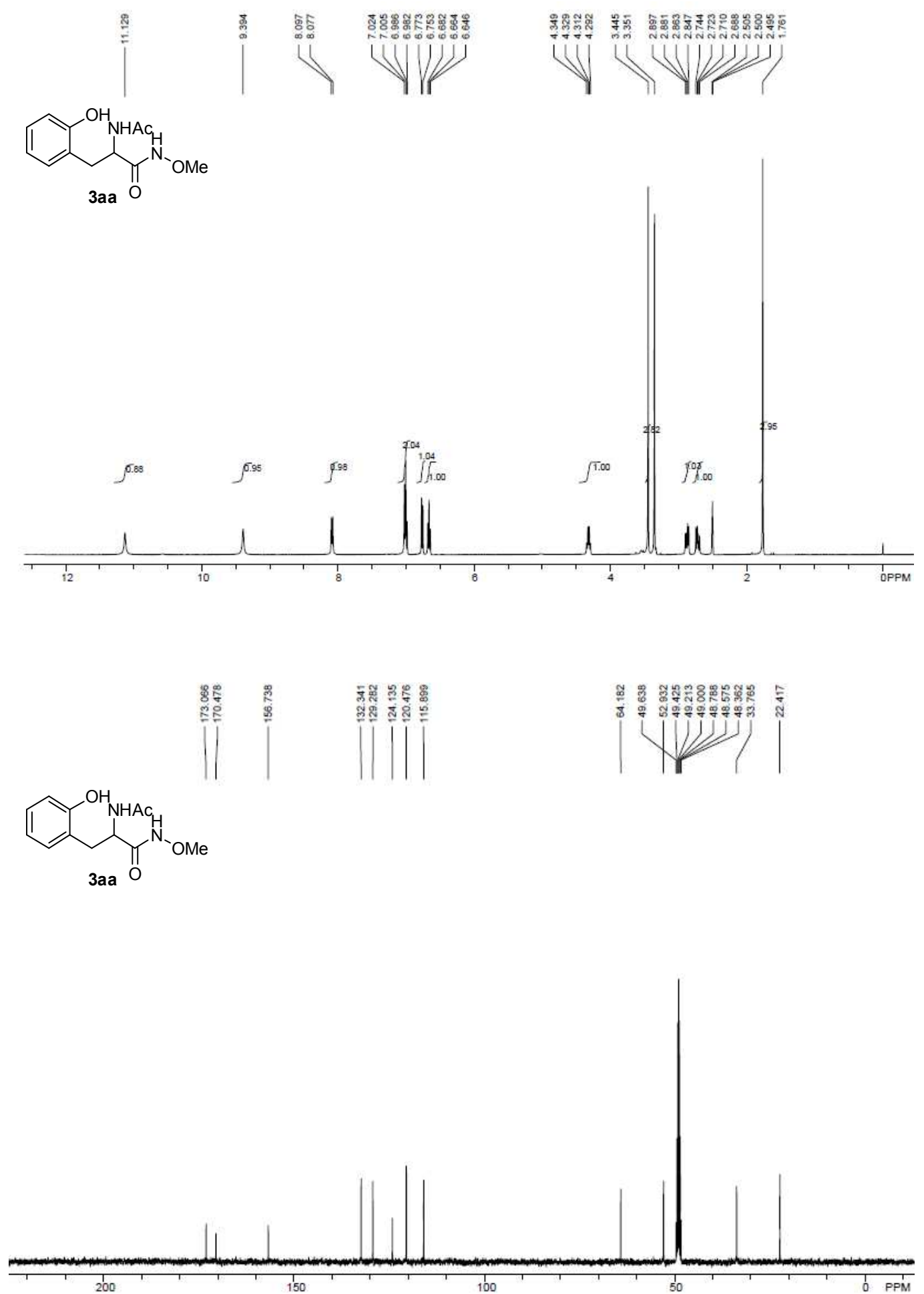

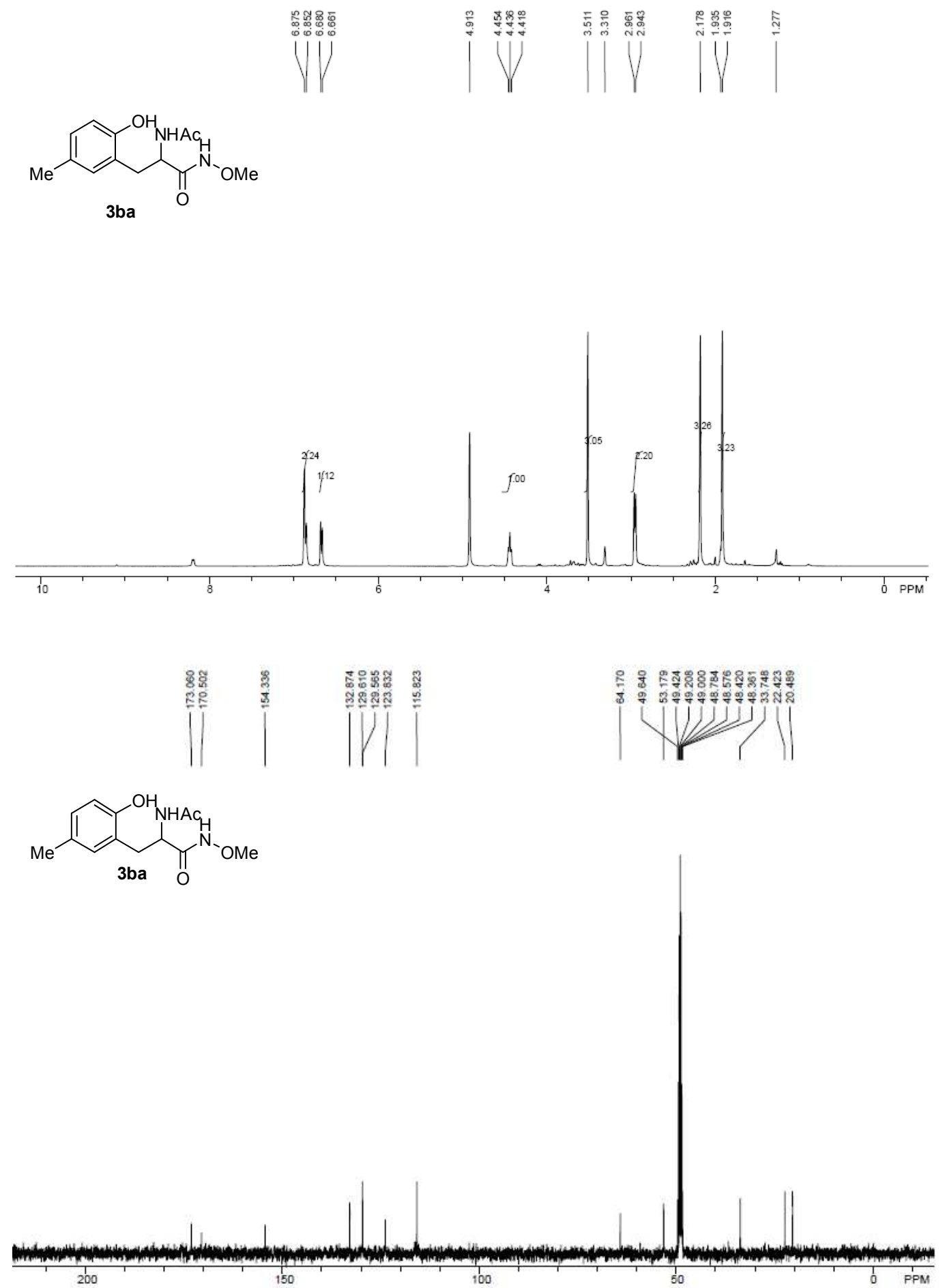

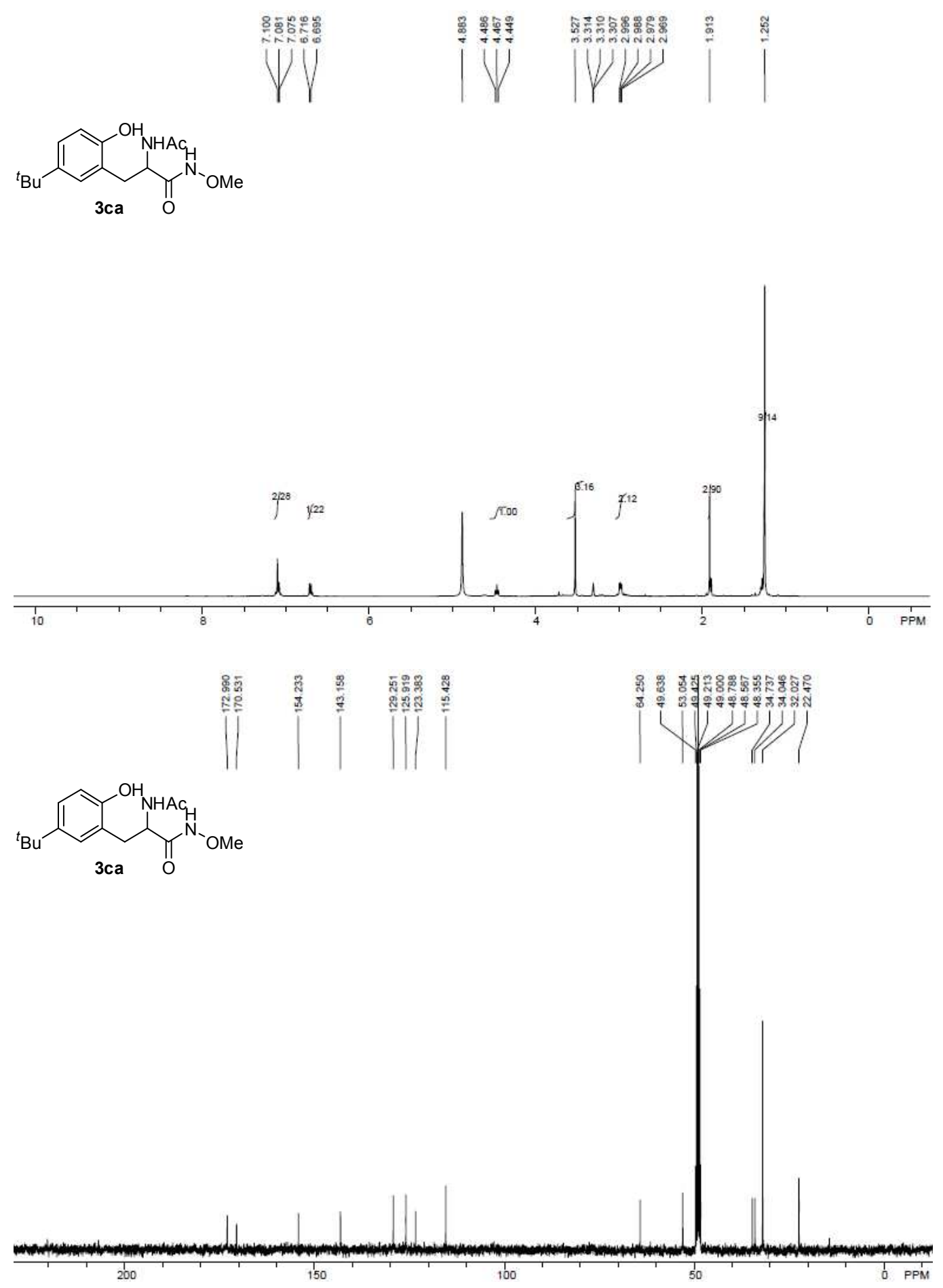

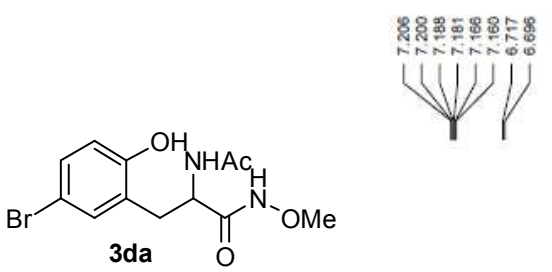

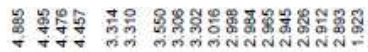

UV U.
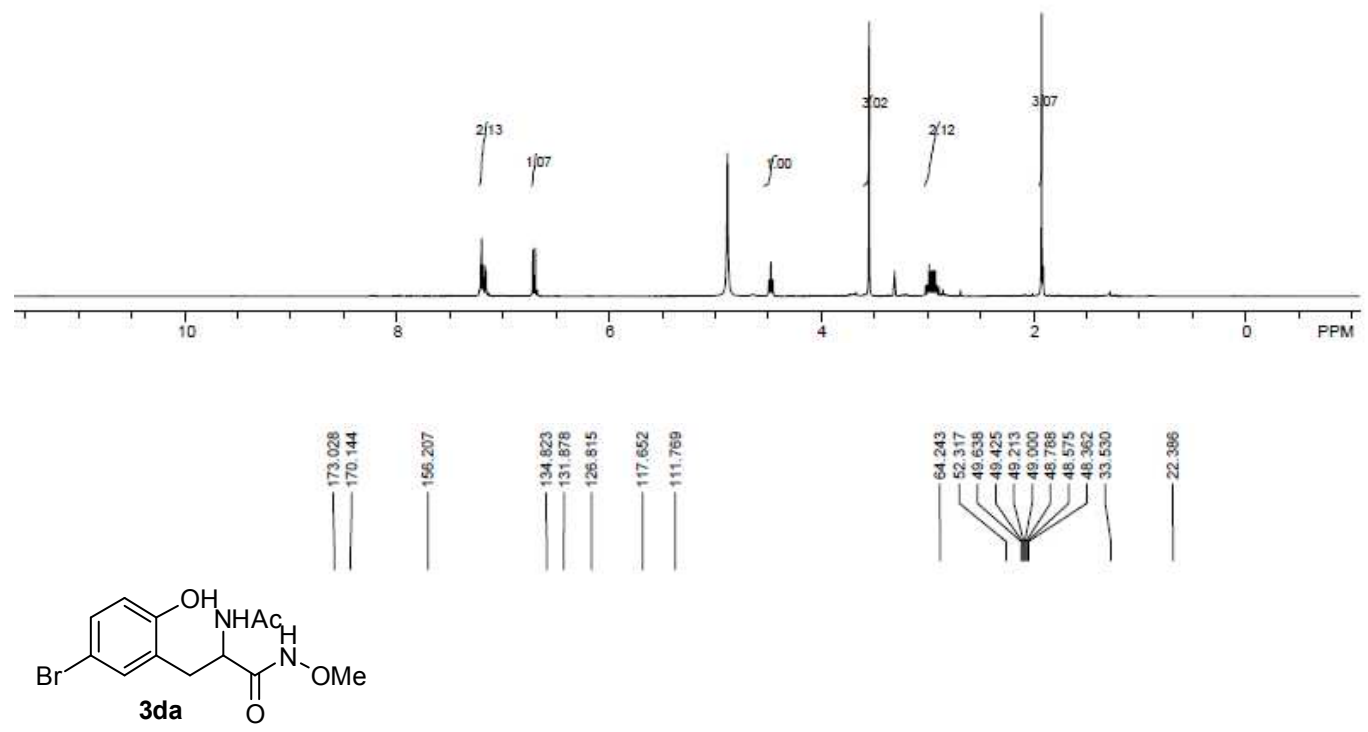

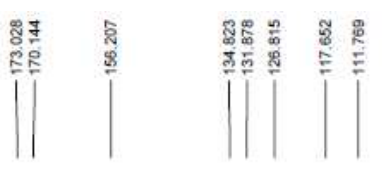
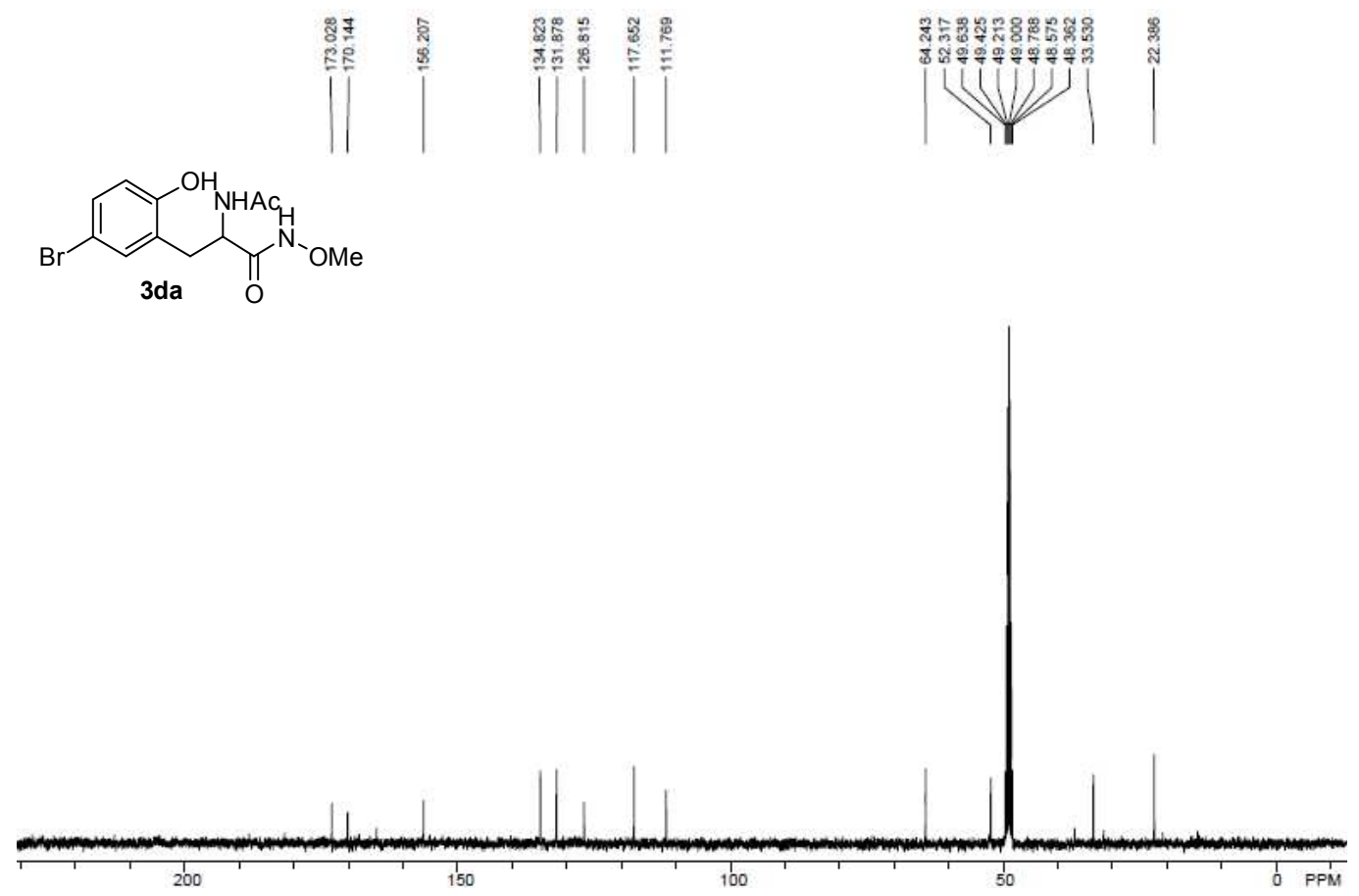

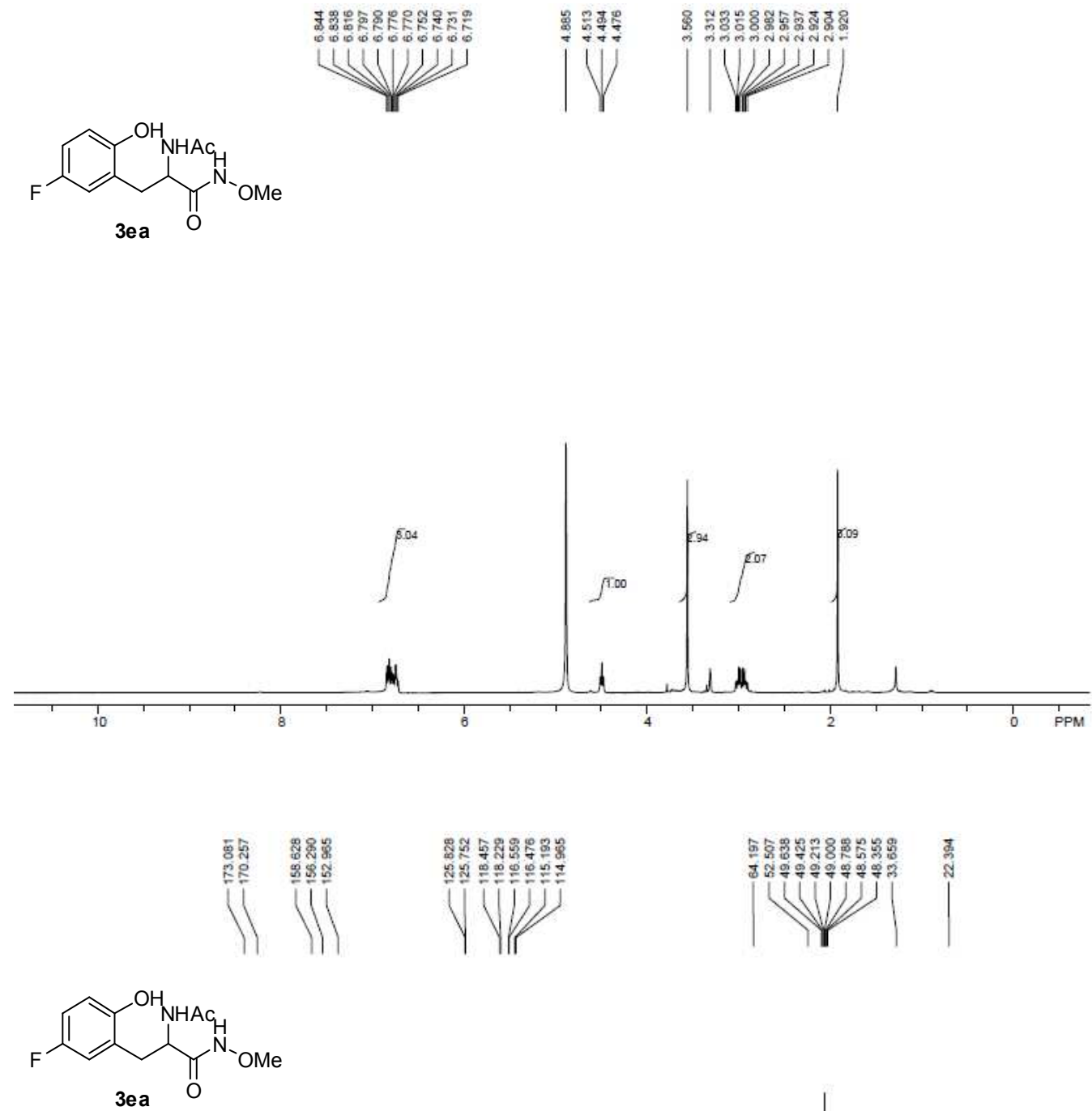

ea

$$
\text { . }
$$



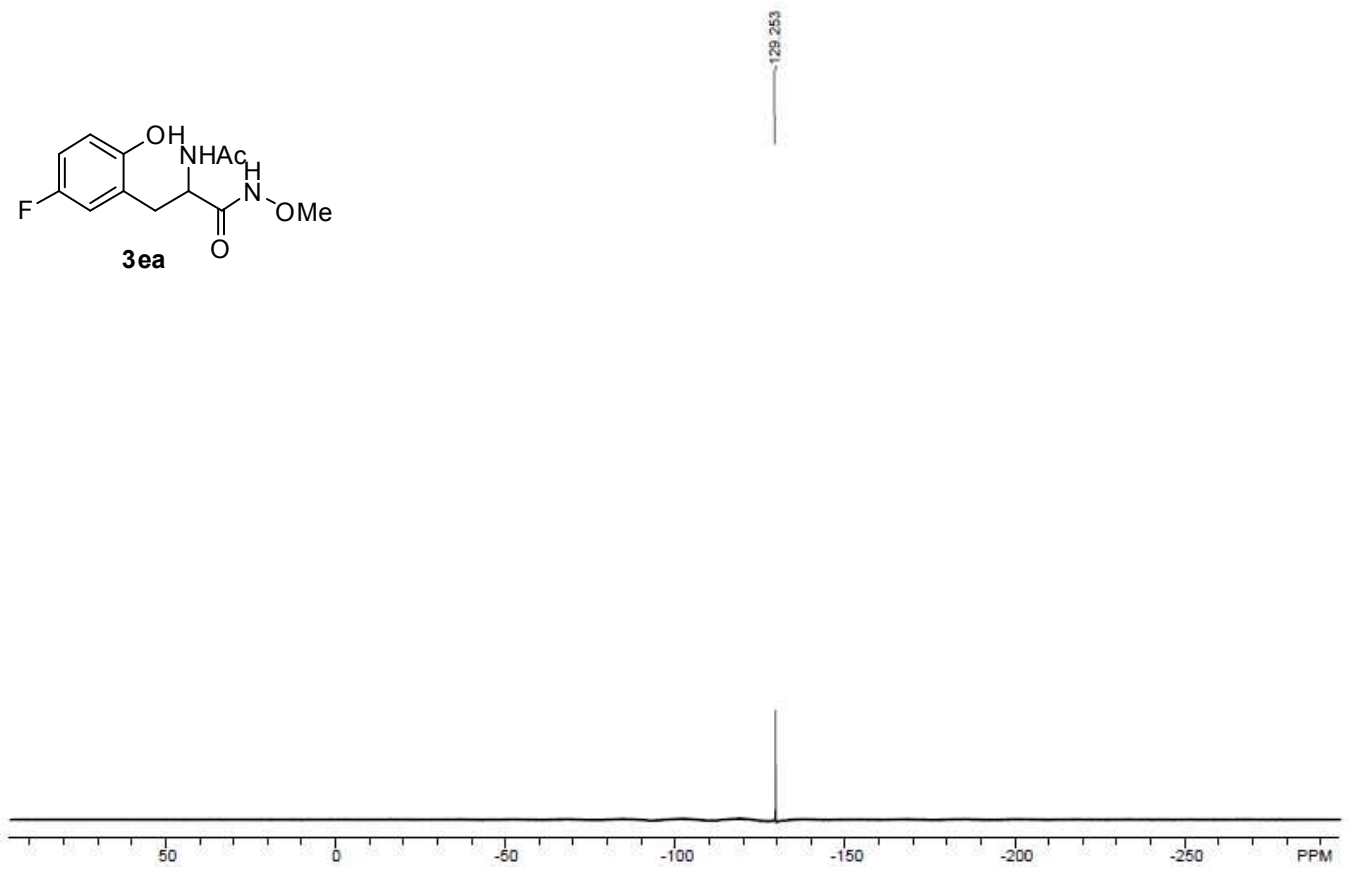

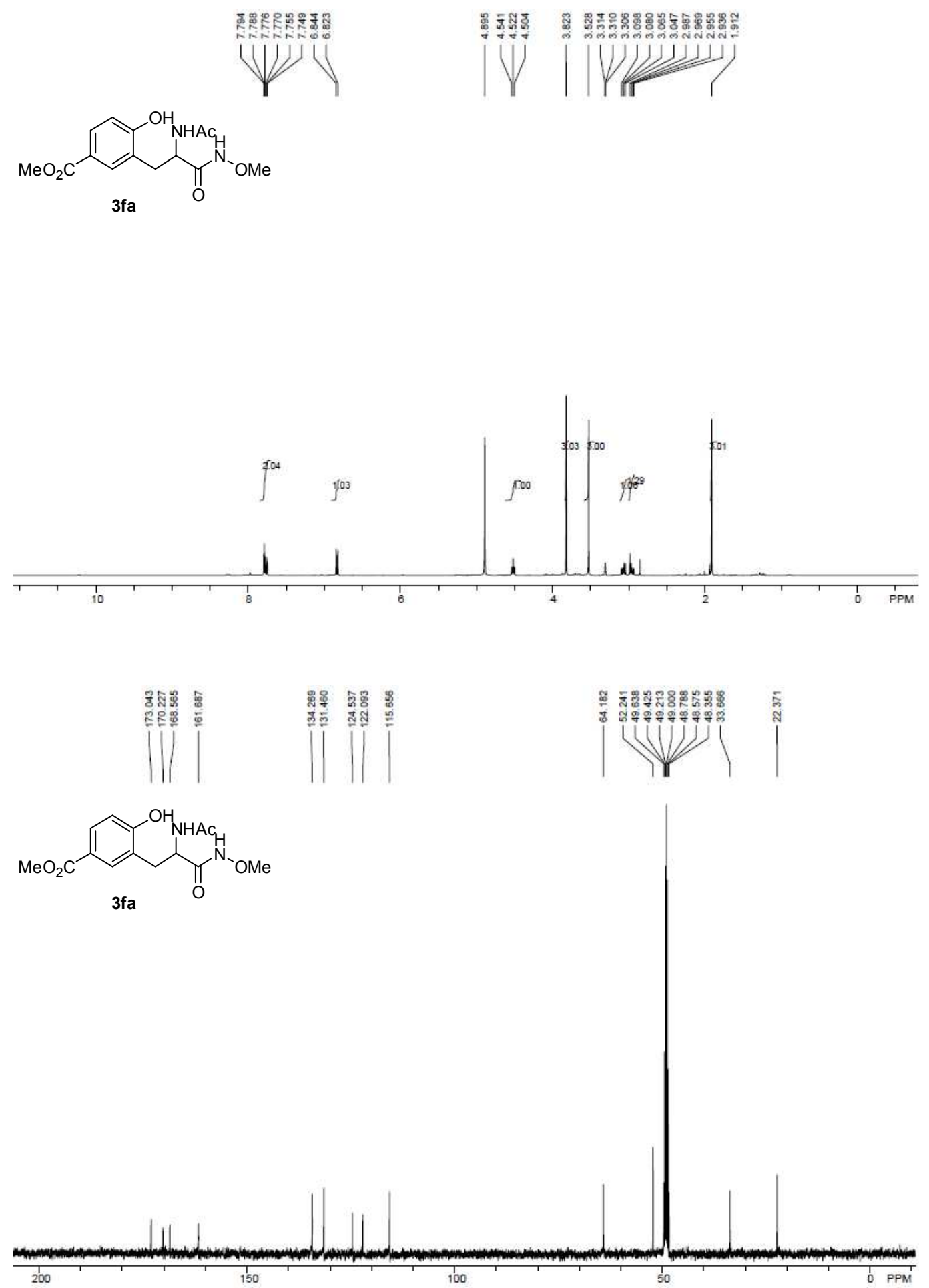

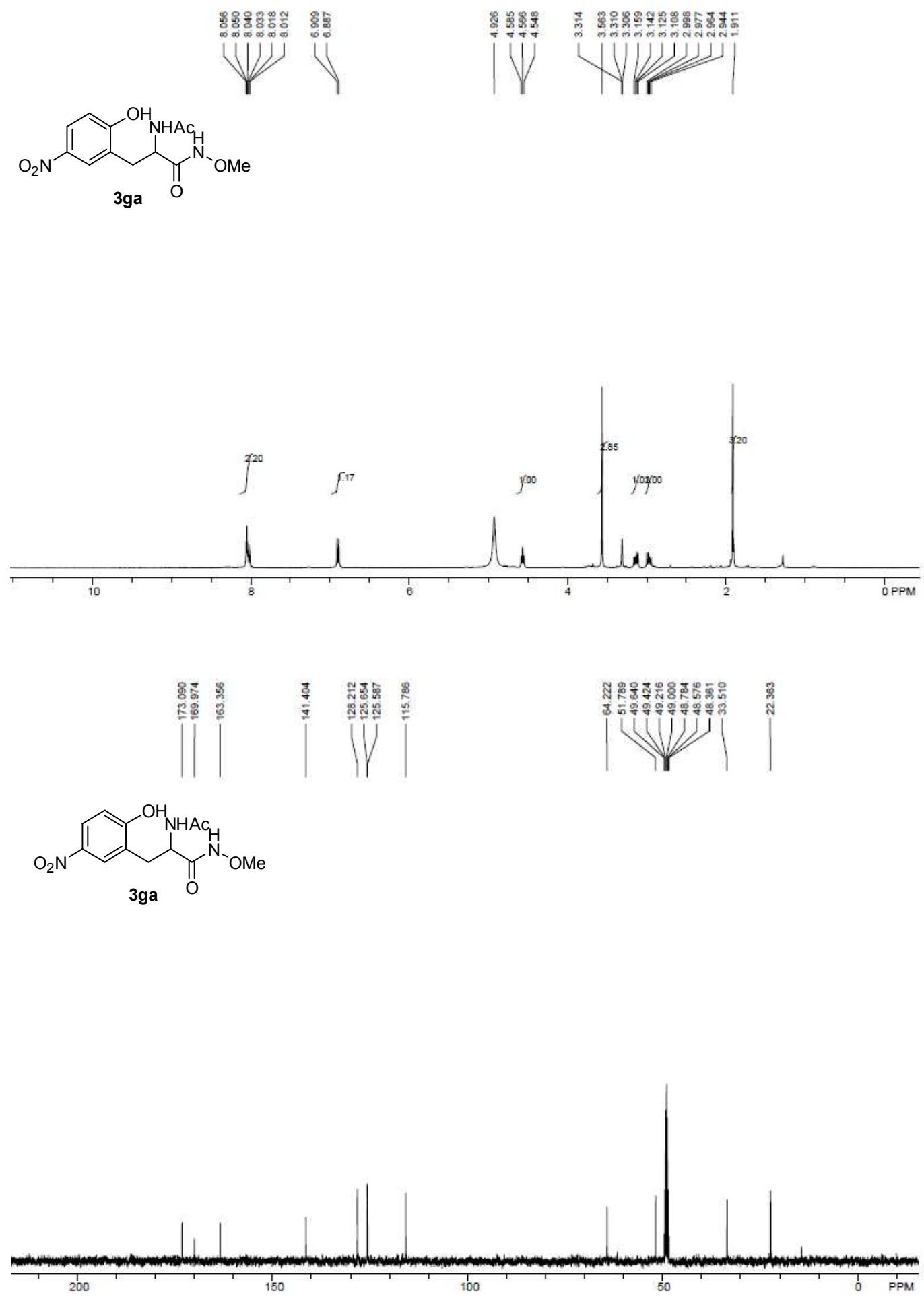

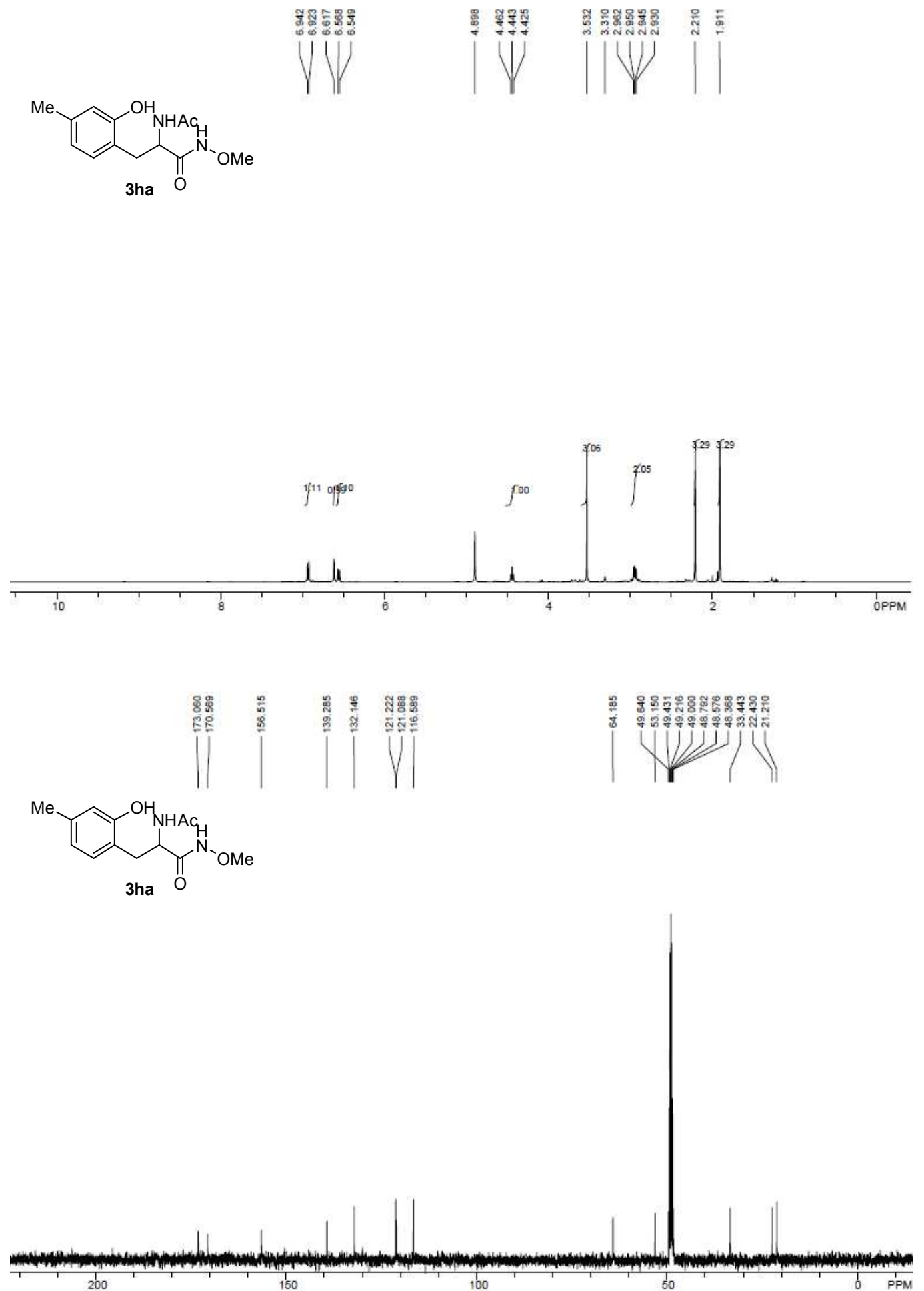

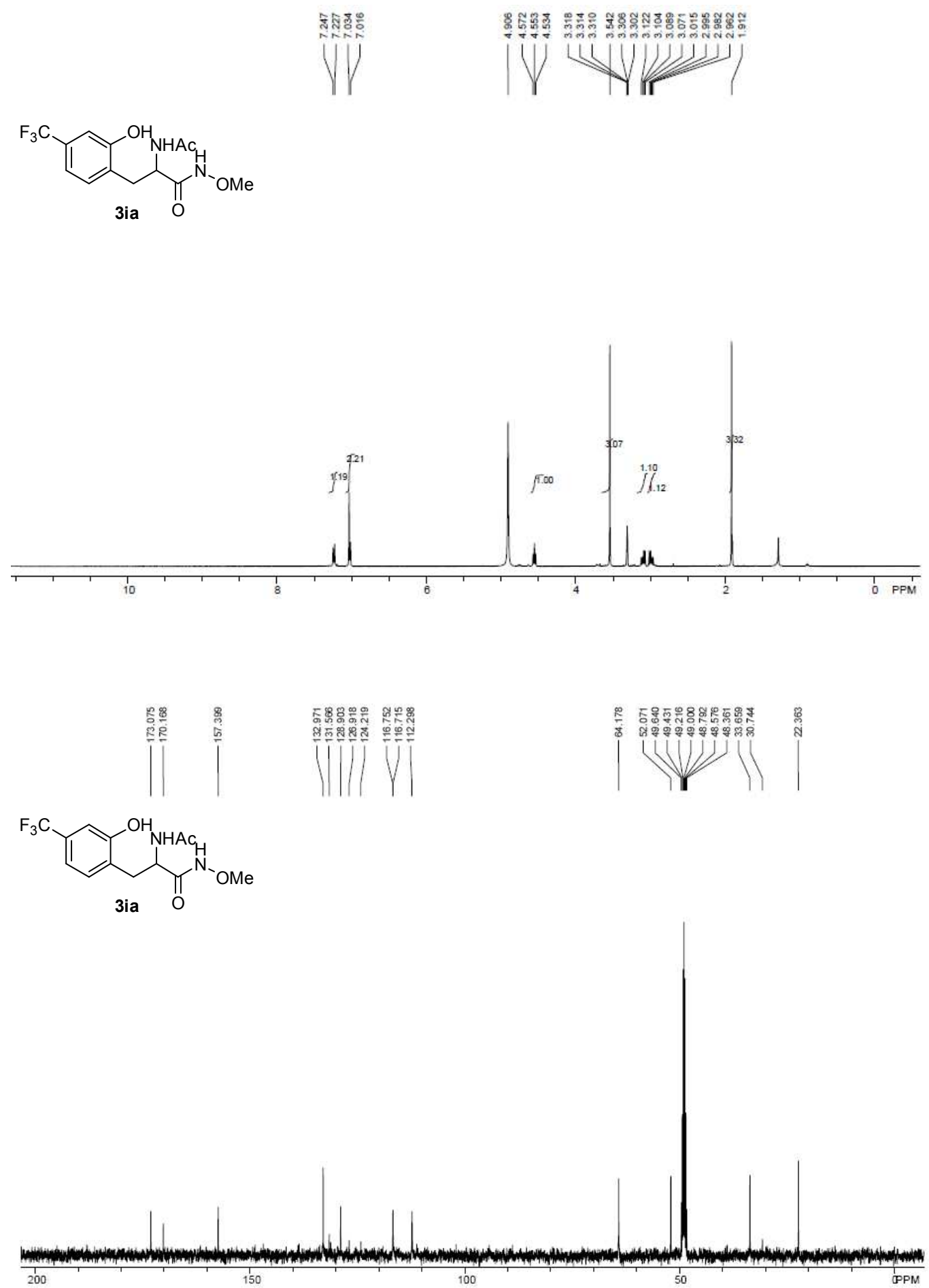

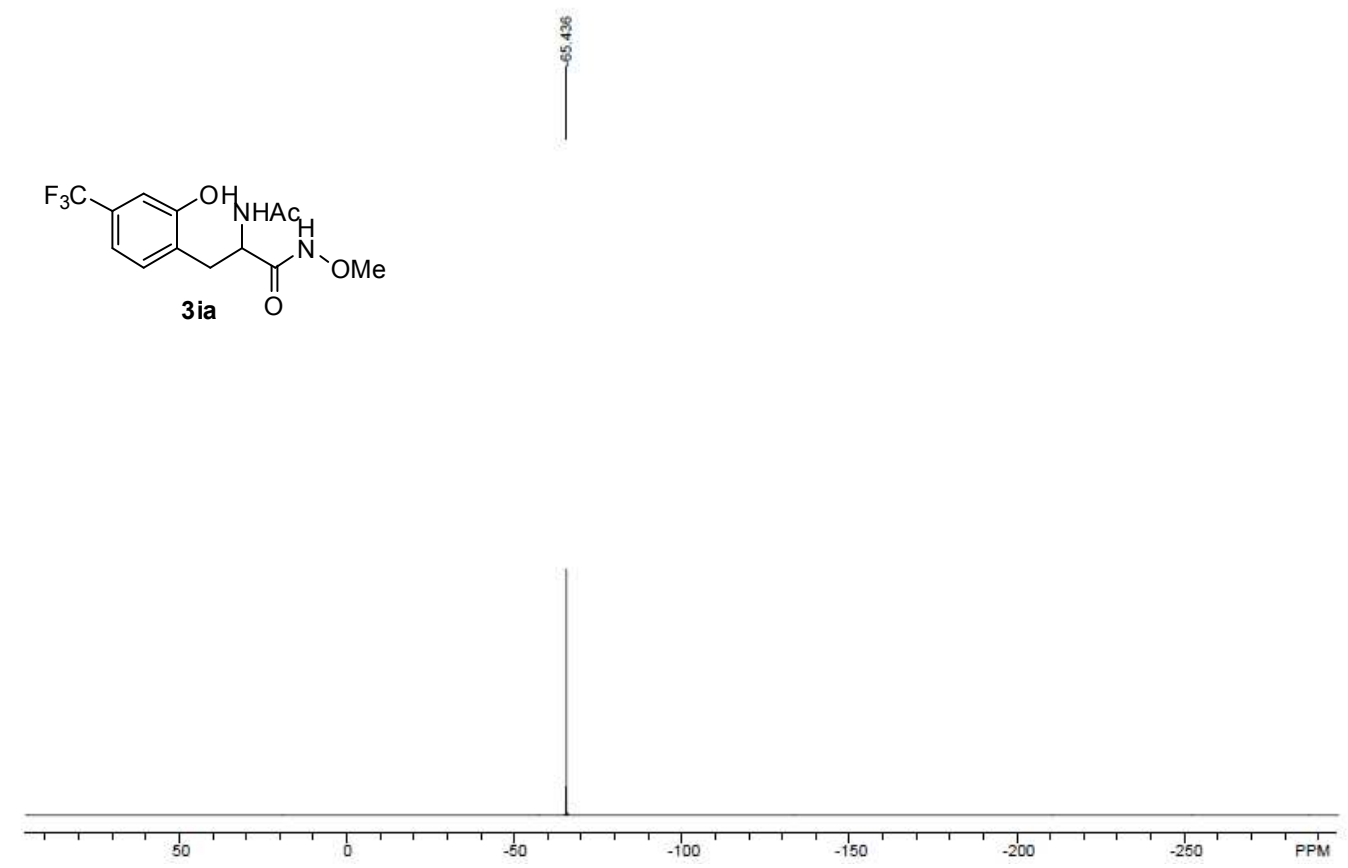

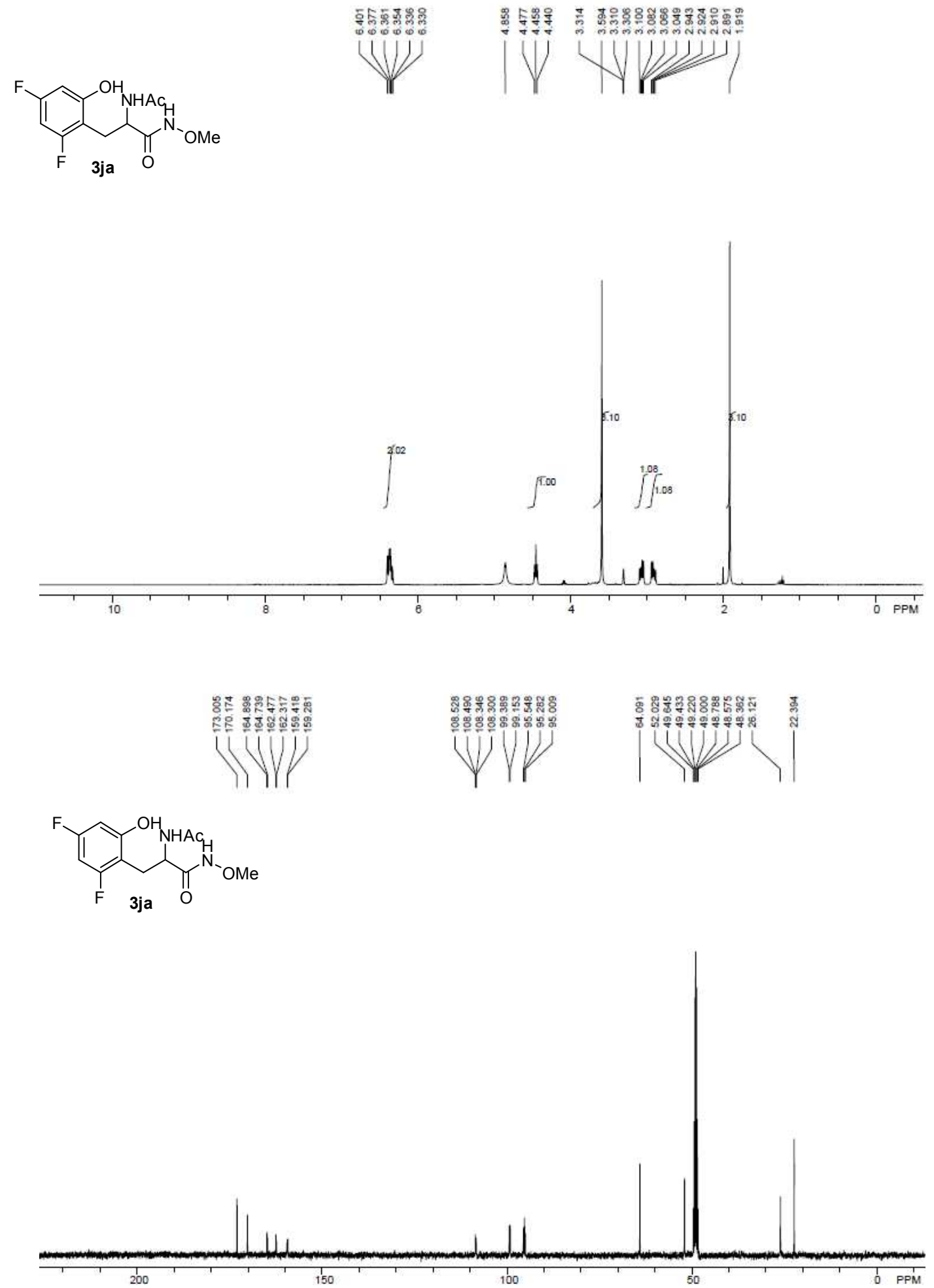

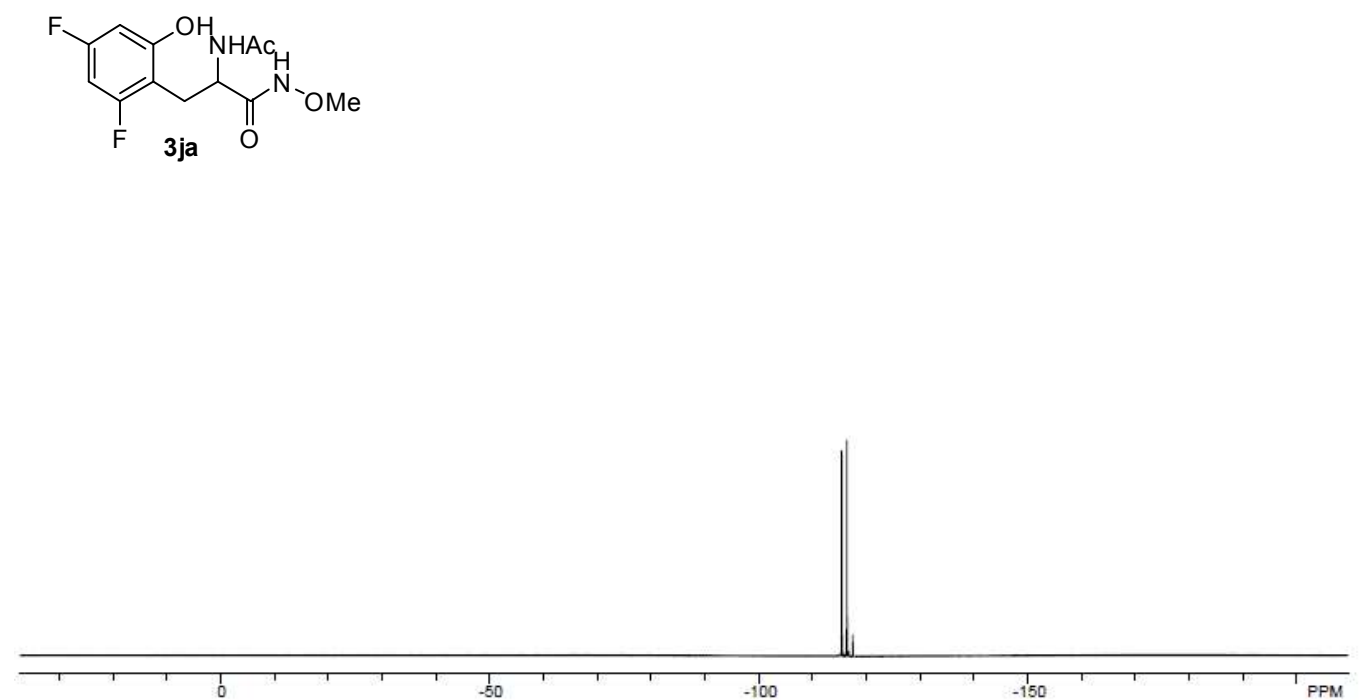
옹정영

(1)

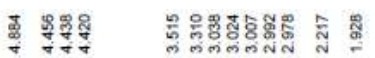

$1 \mathrm{~W}|\mathrm{~W}|$
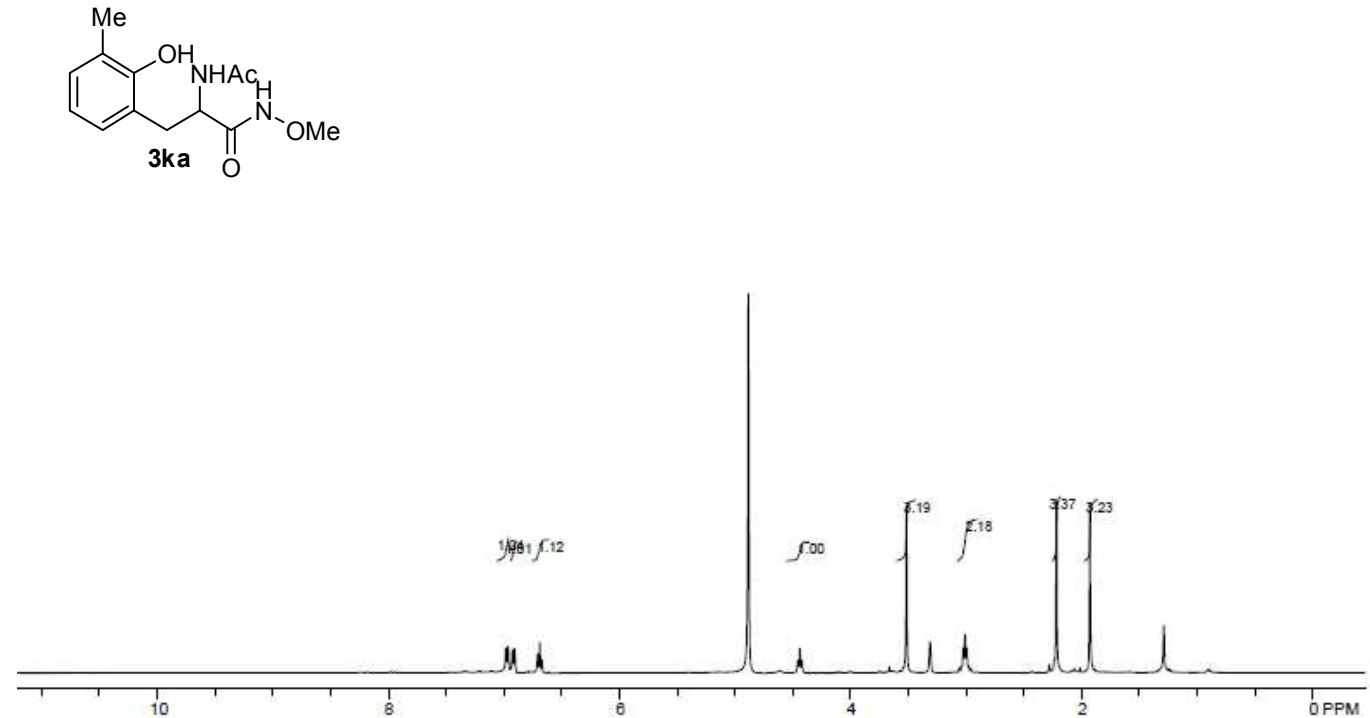

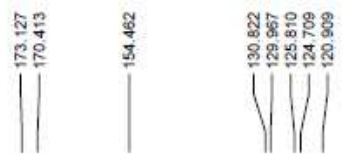
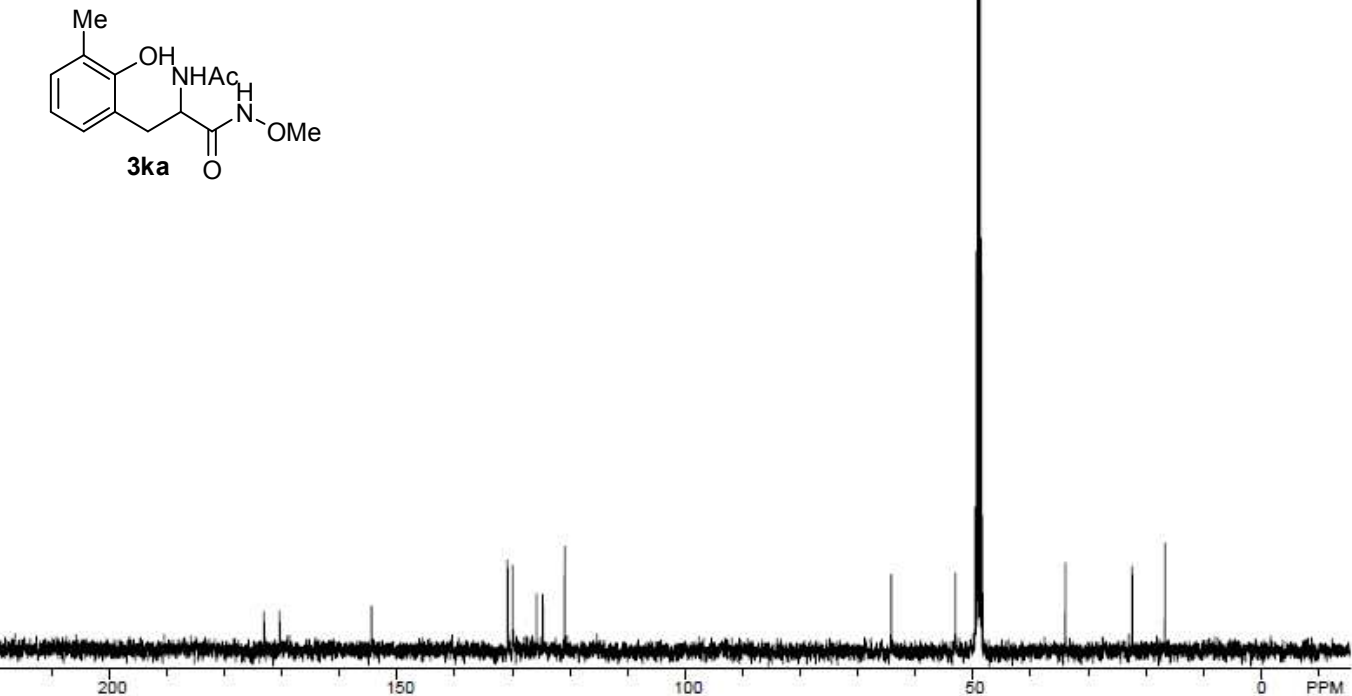


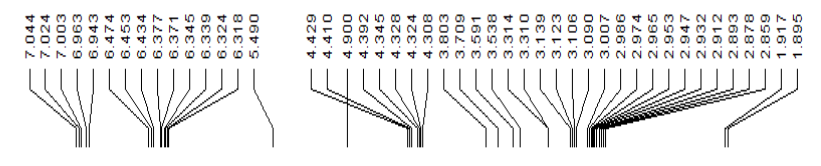

3la $\mathrm{O}_{\mathrm{OMe}}^{\mathrm{Mla} \mathrm{O}_{\mathrm{O}}}$
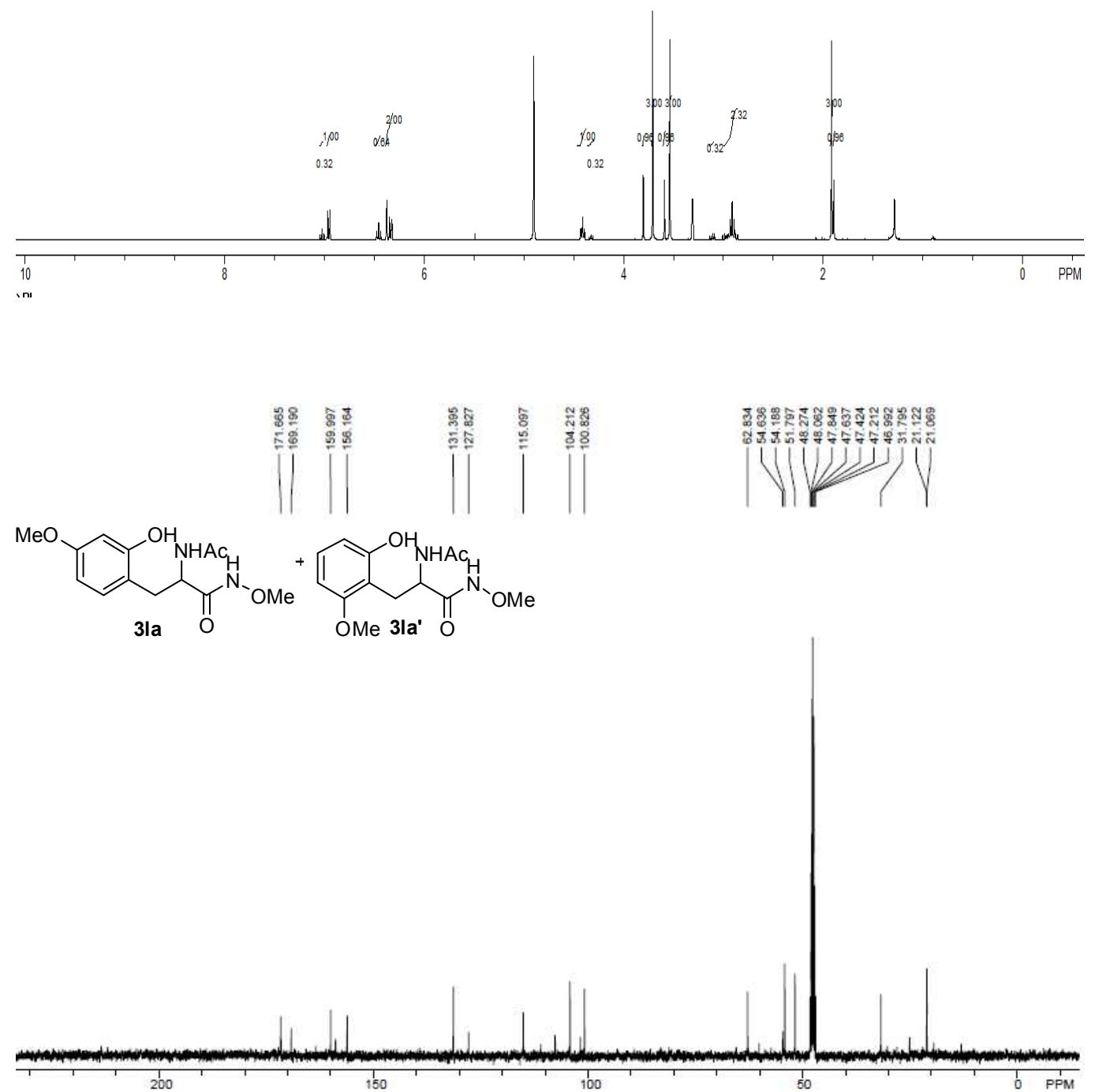

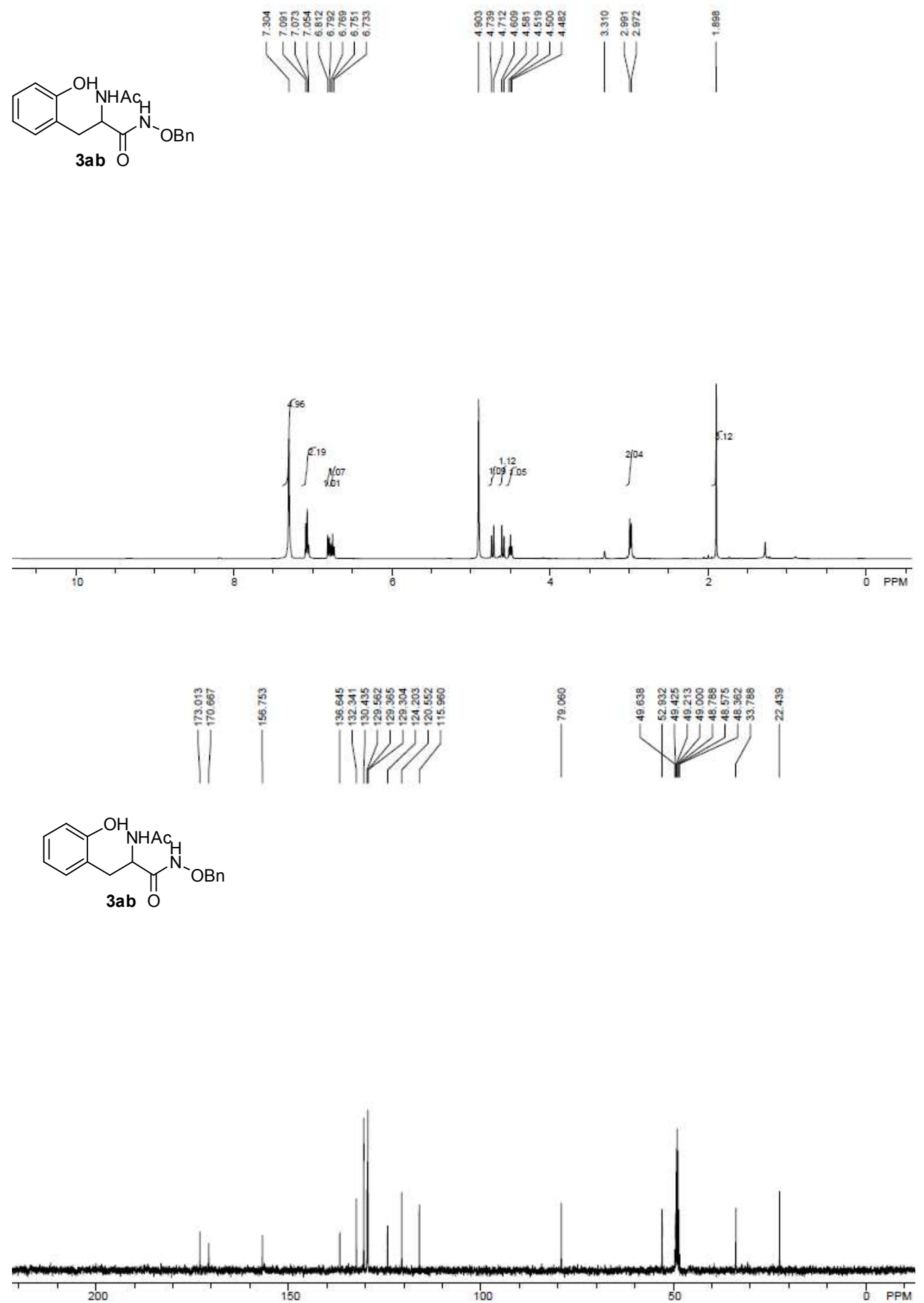

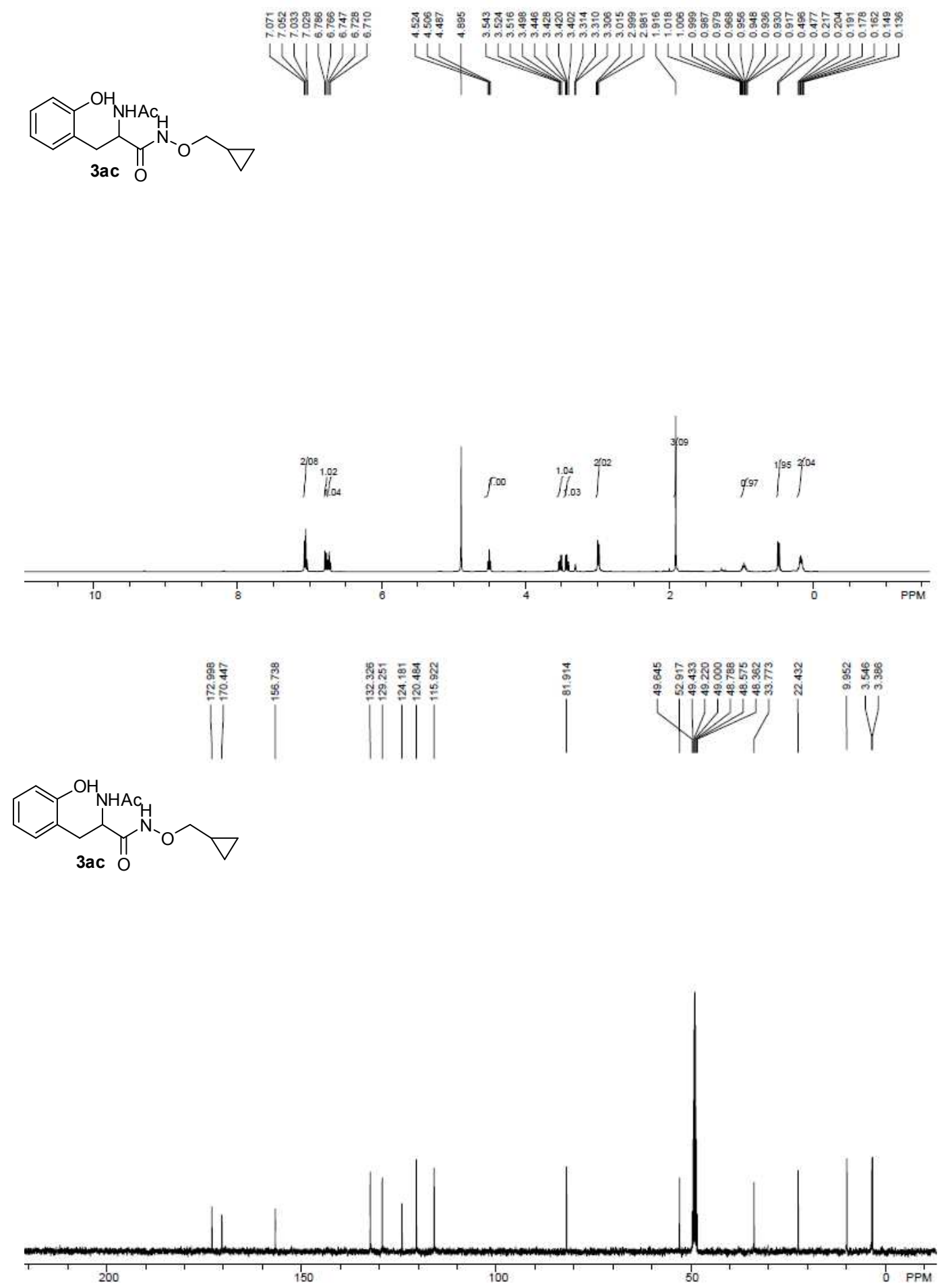

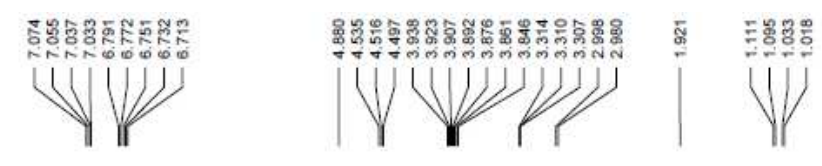

$\underbrace{O}_{3 a d}$
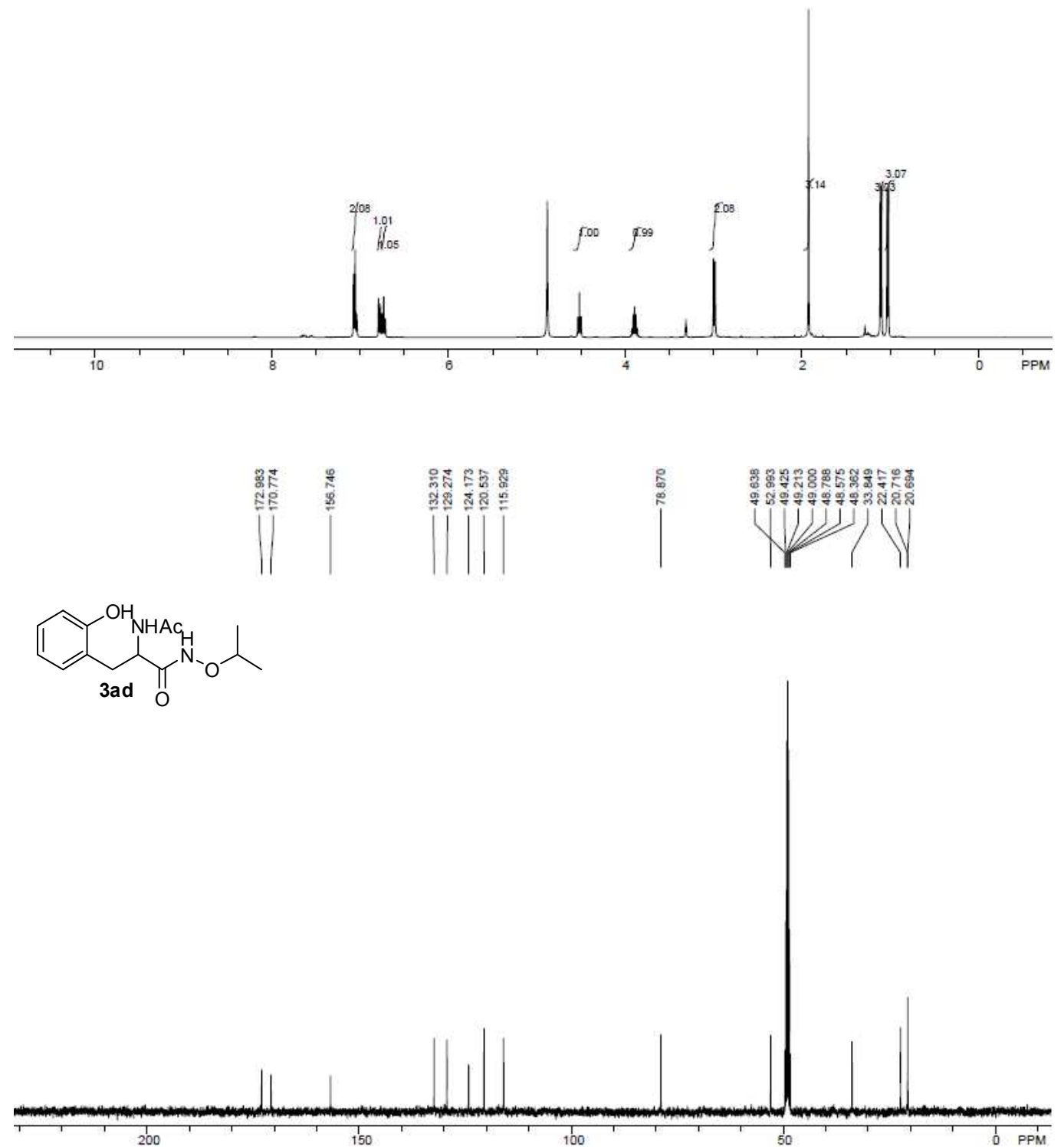

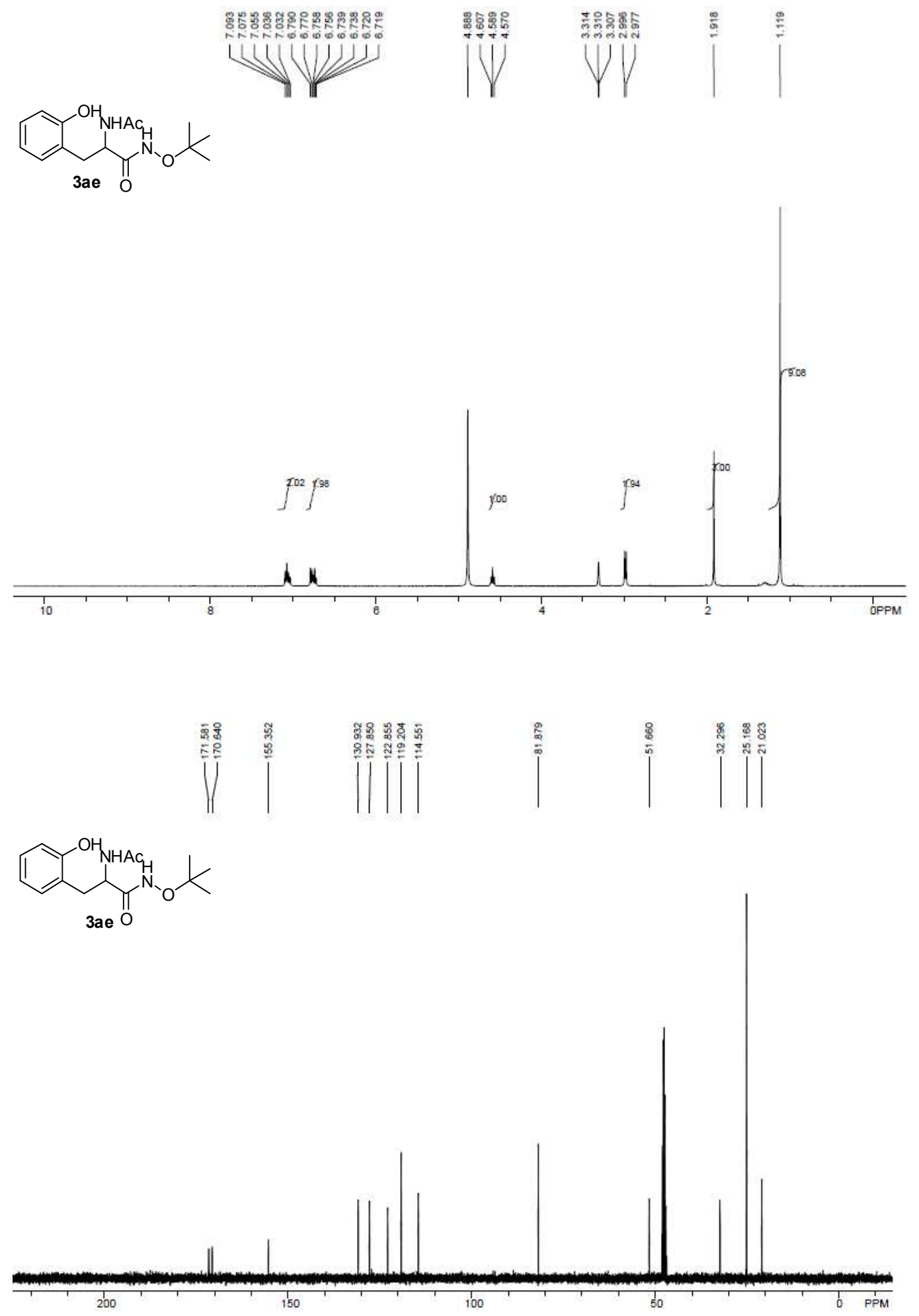

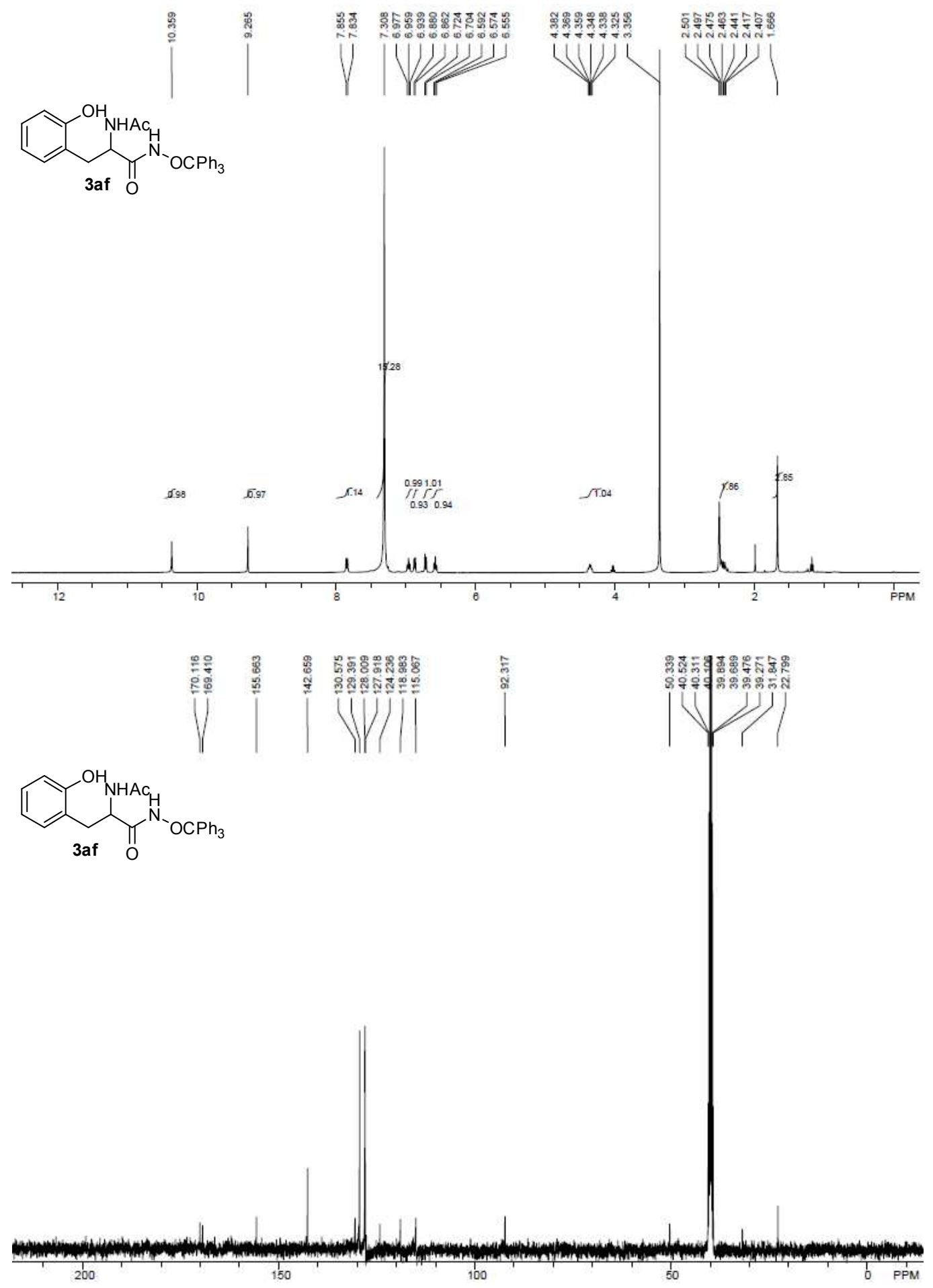

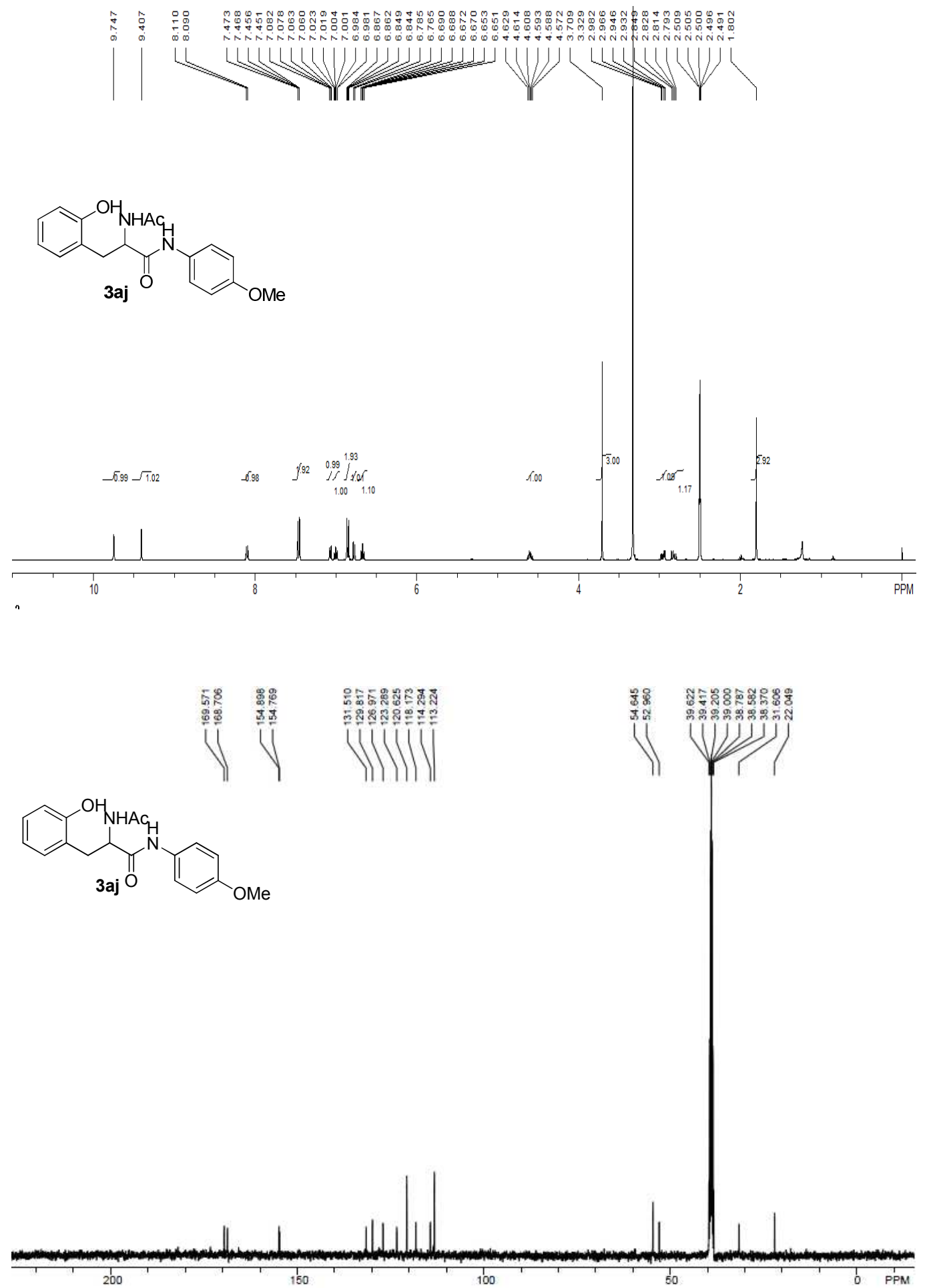

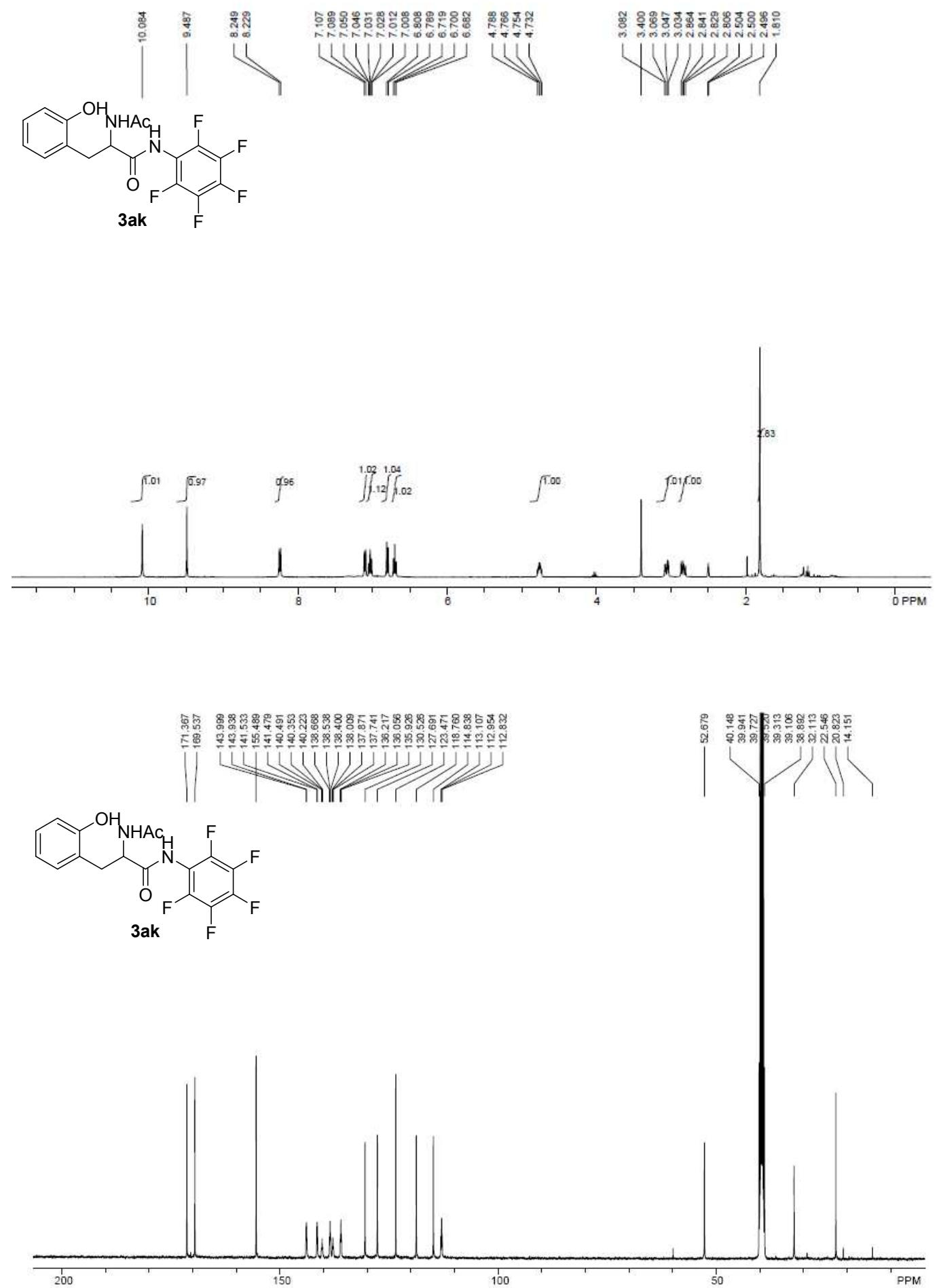

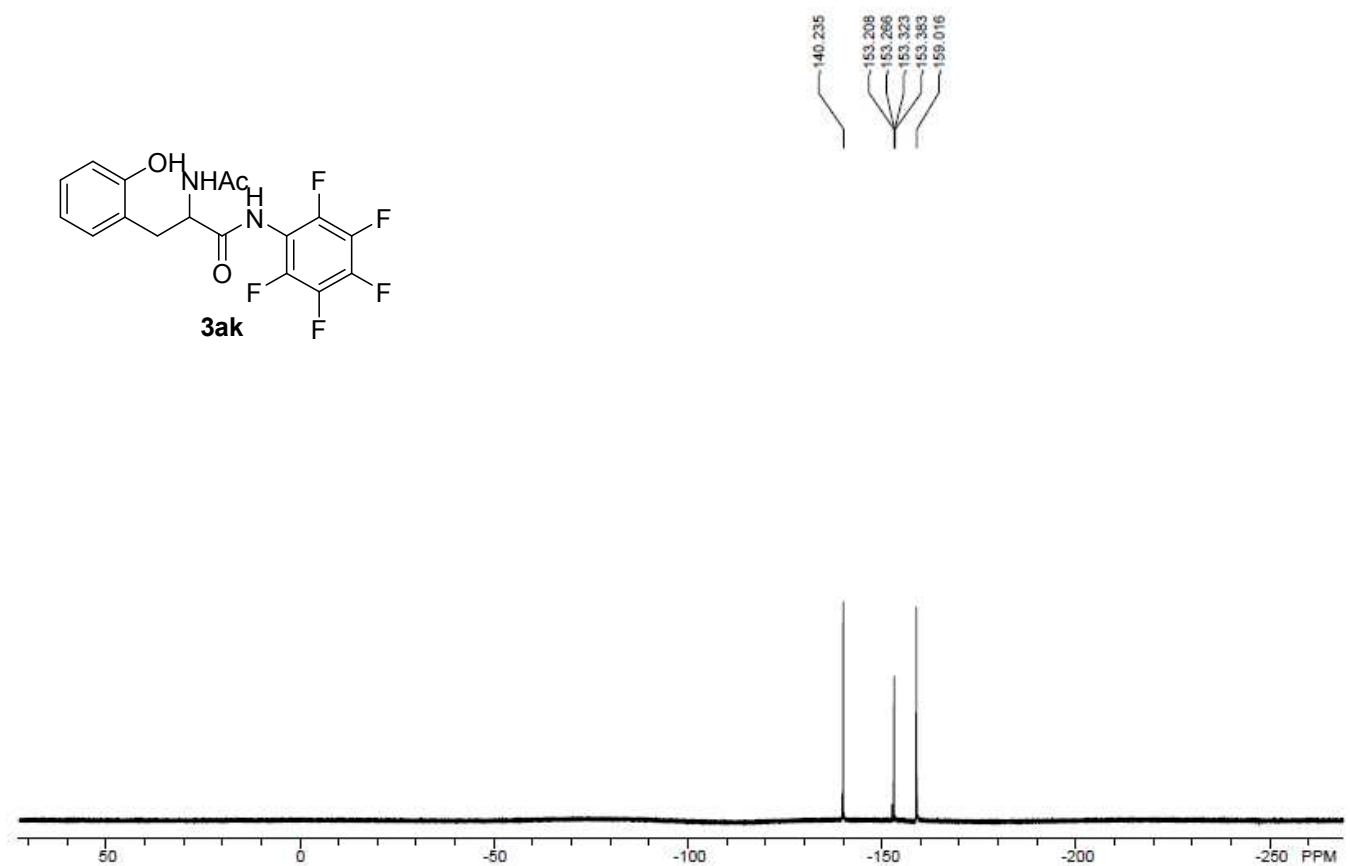


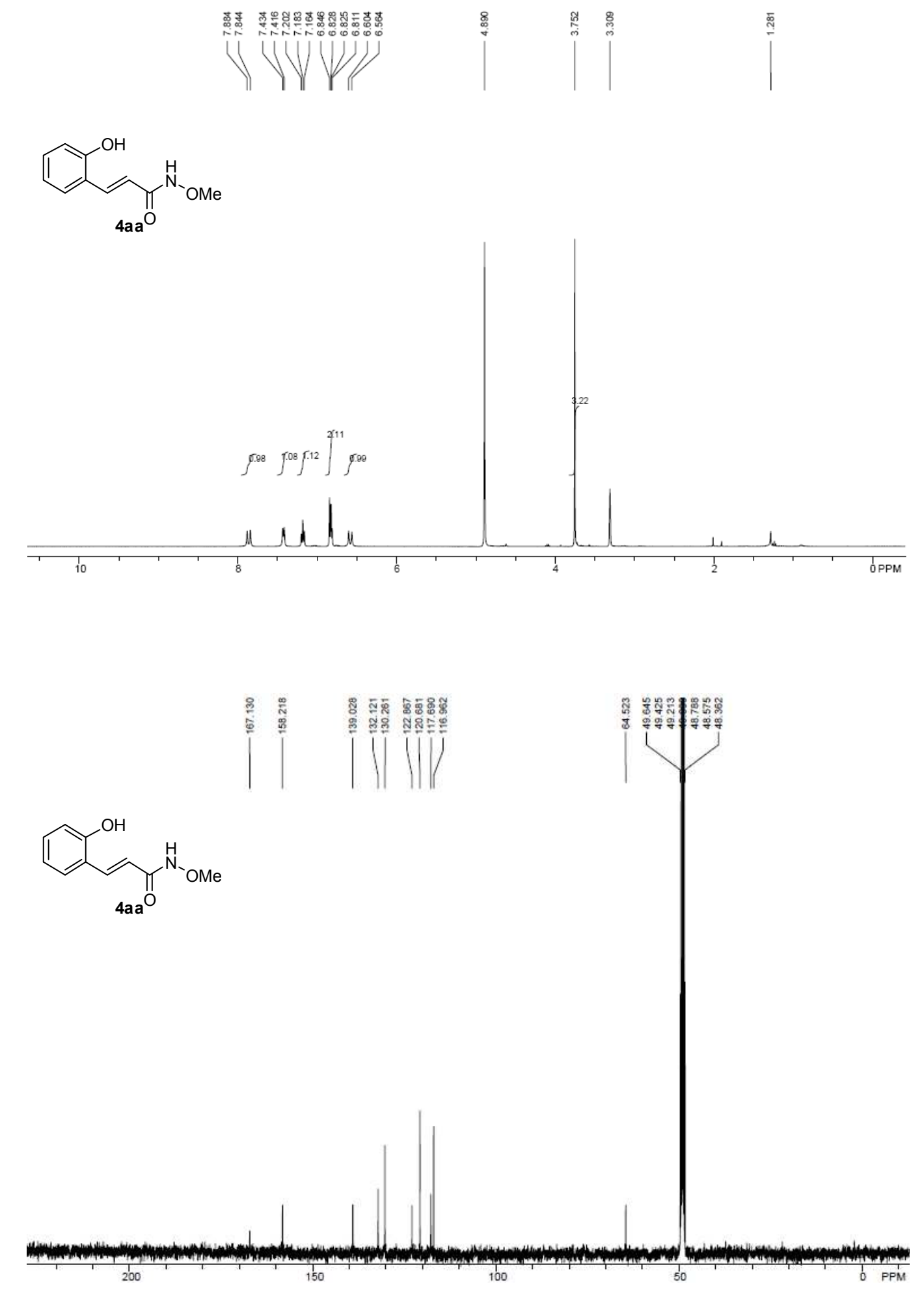



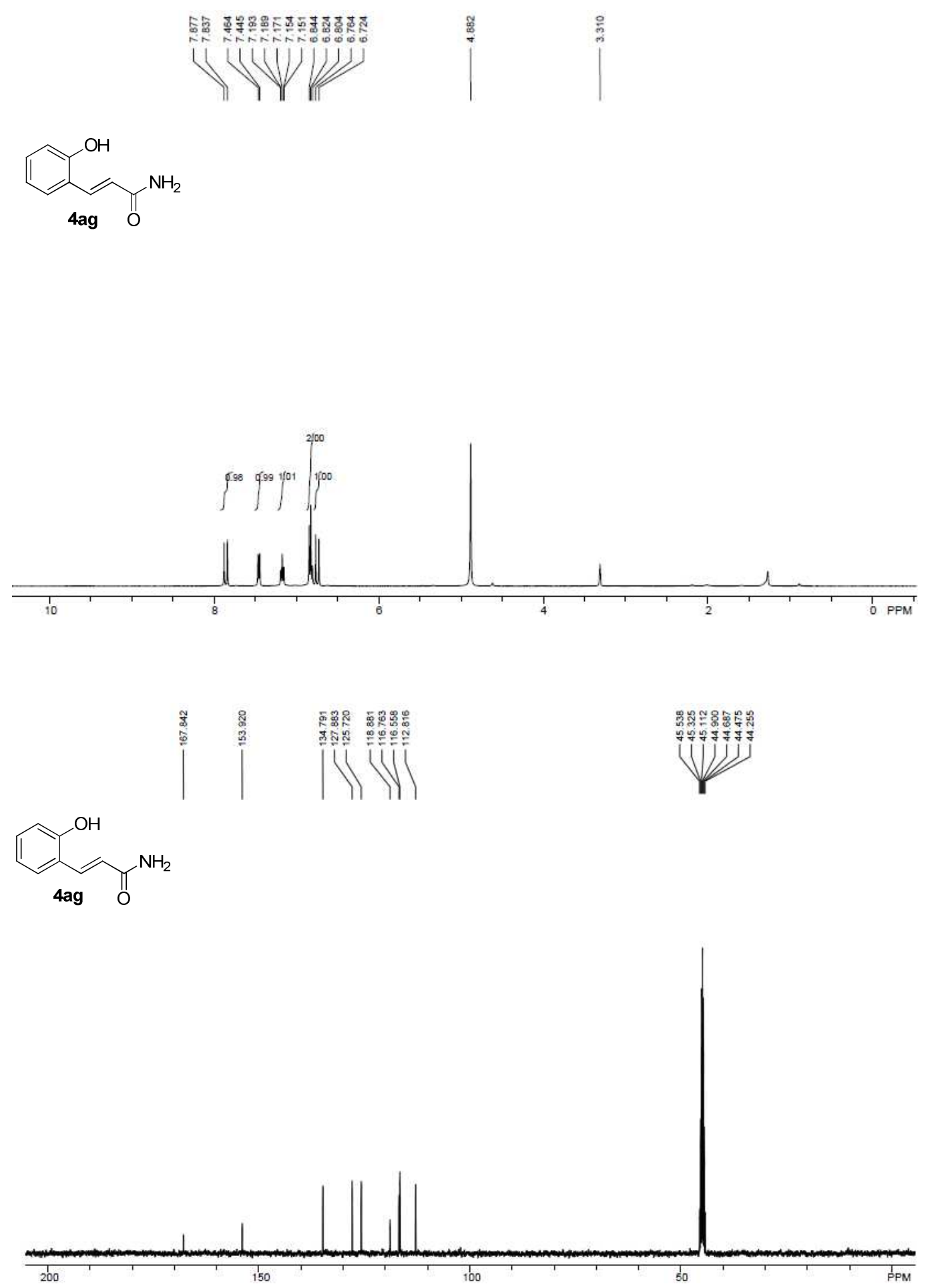

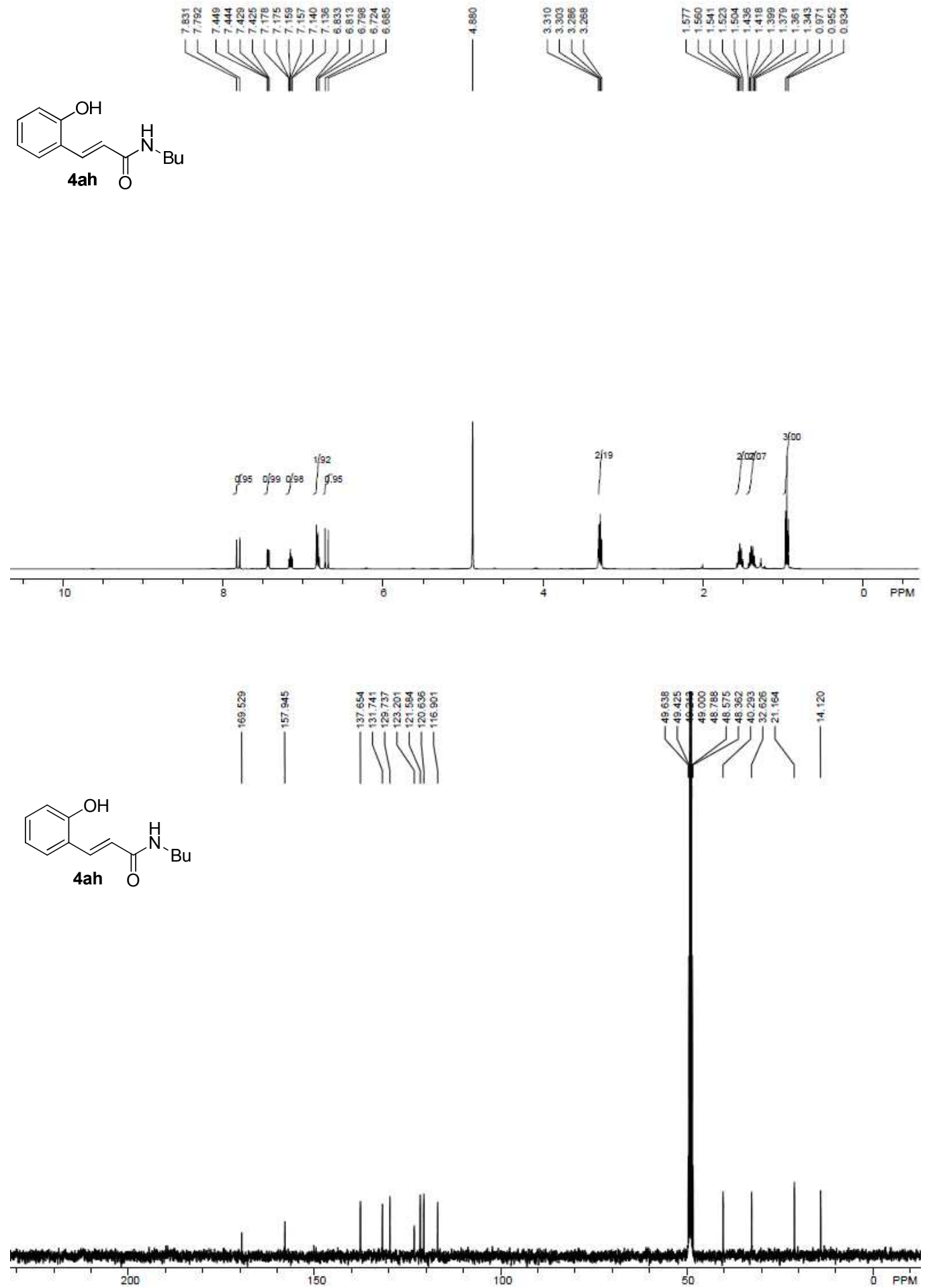

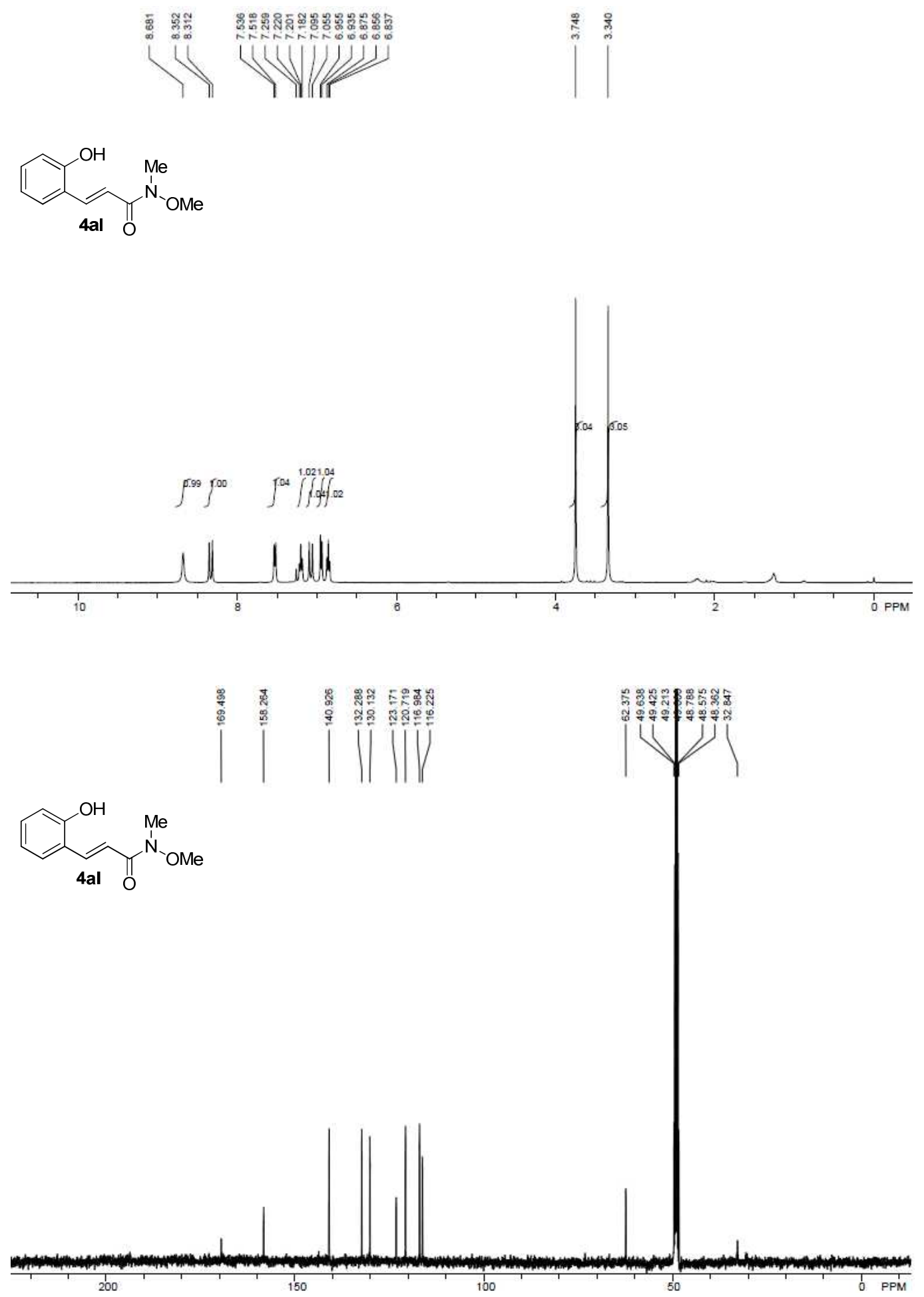

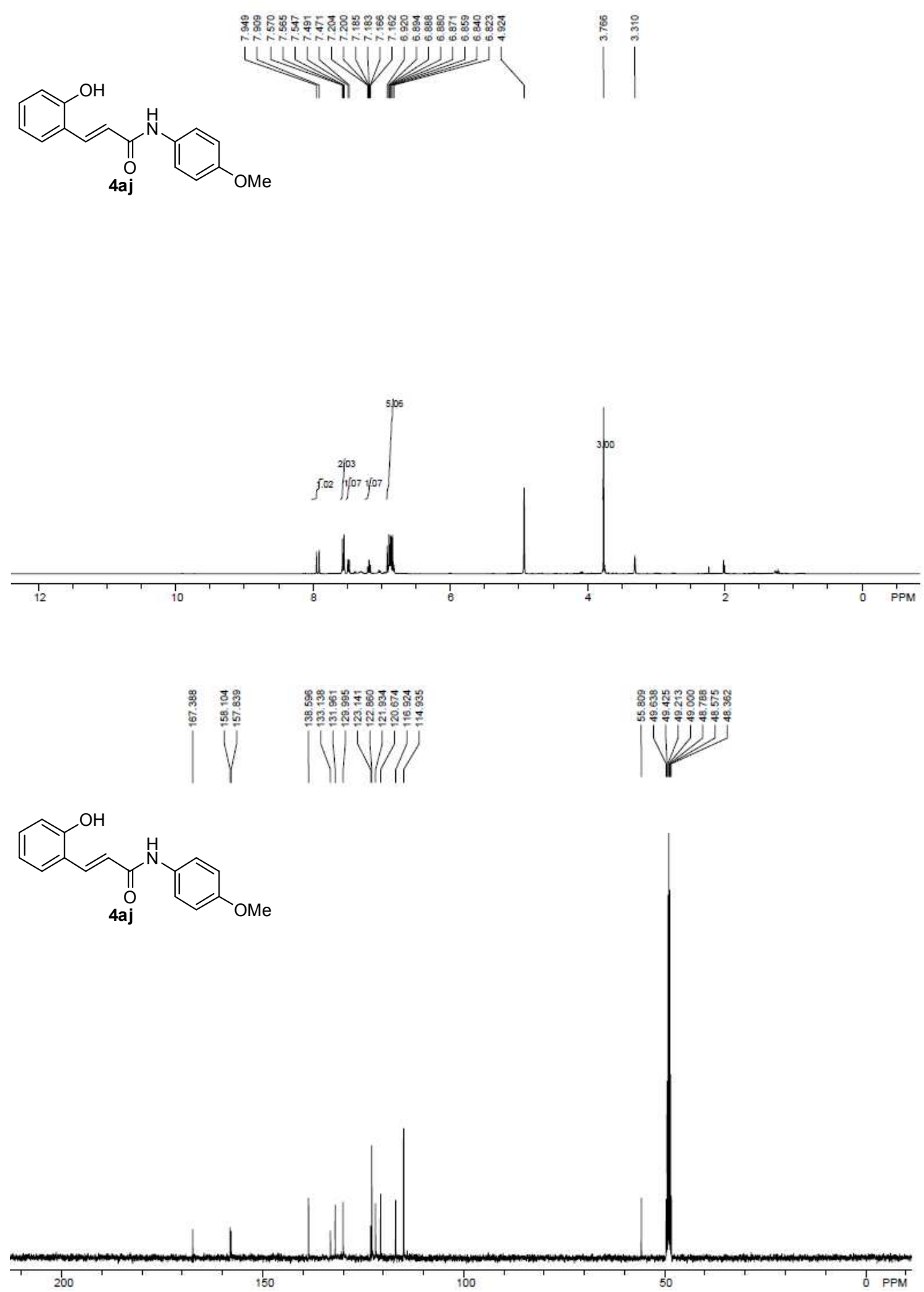

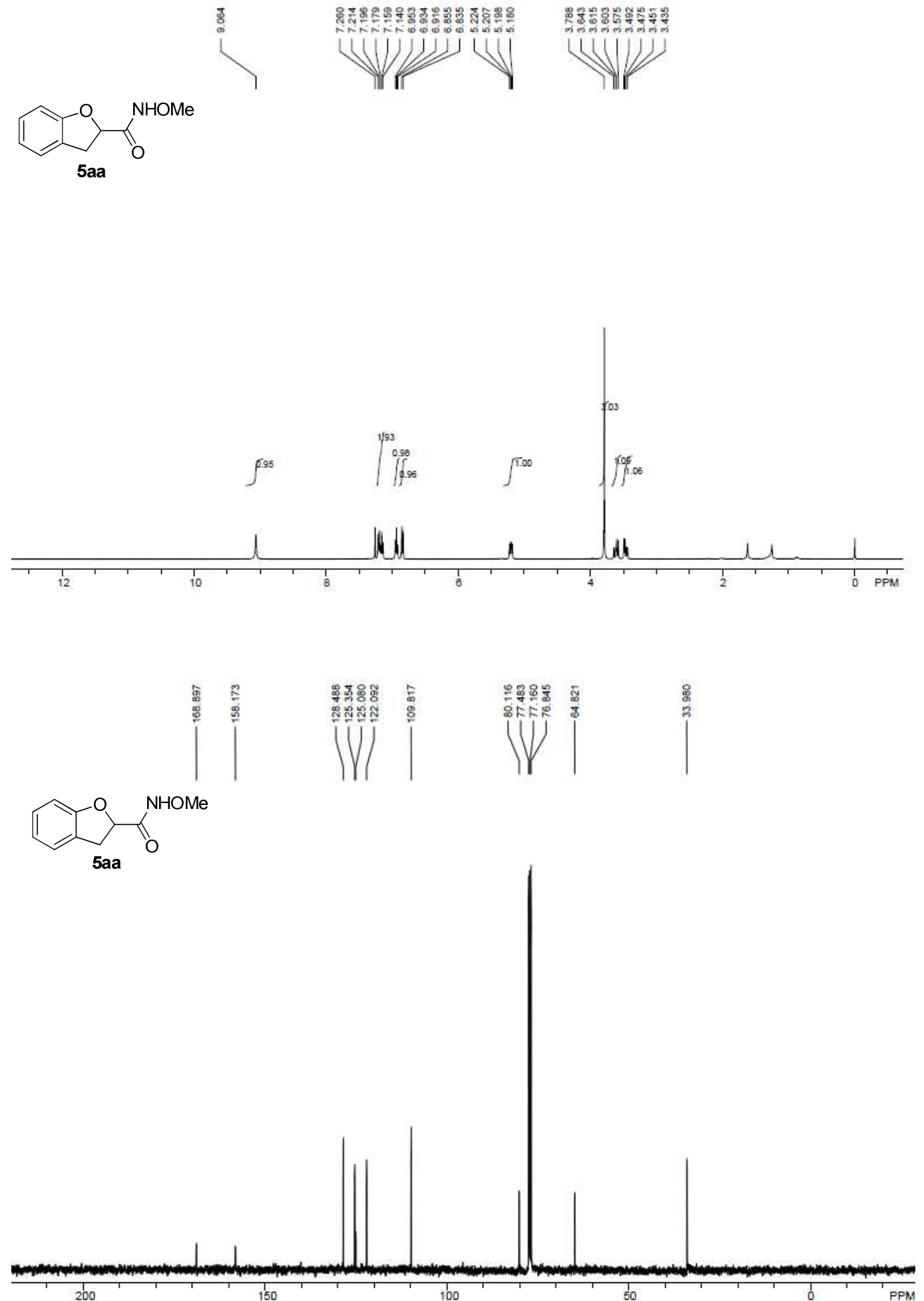

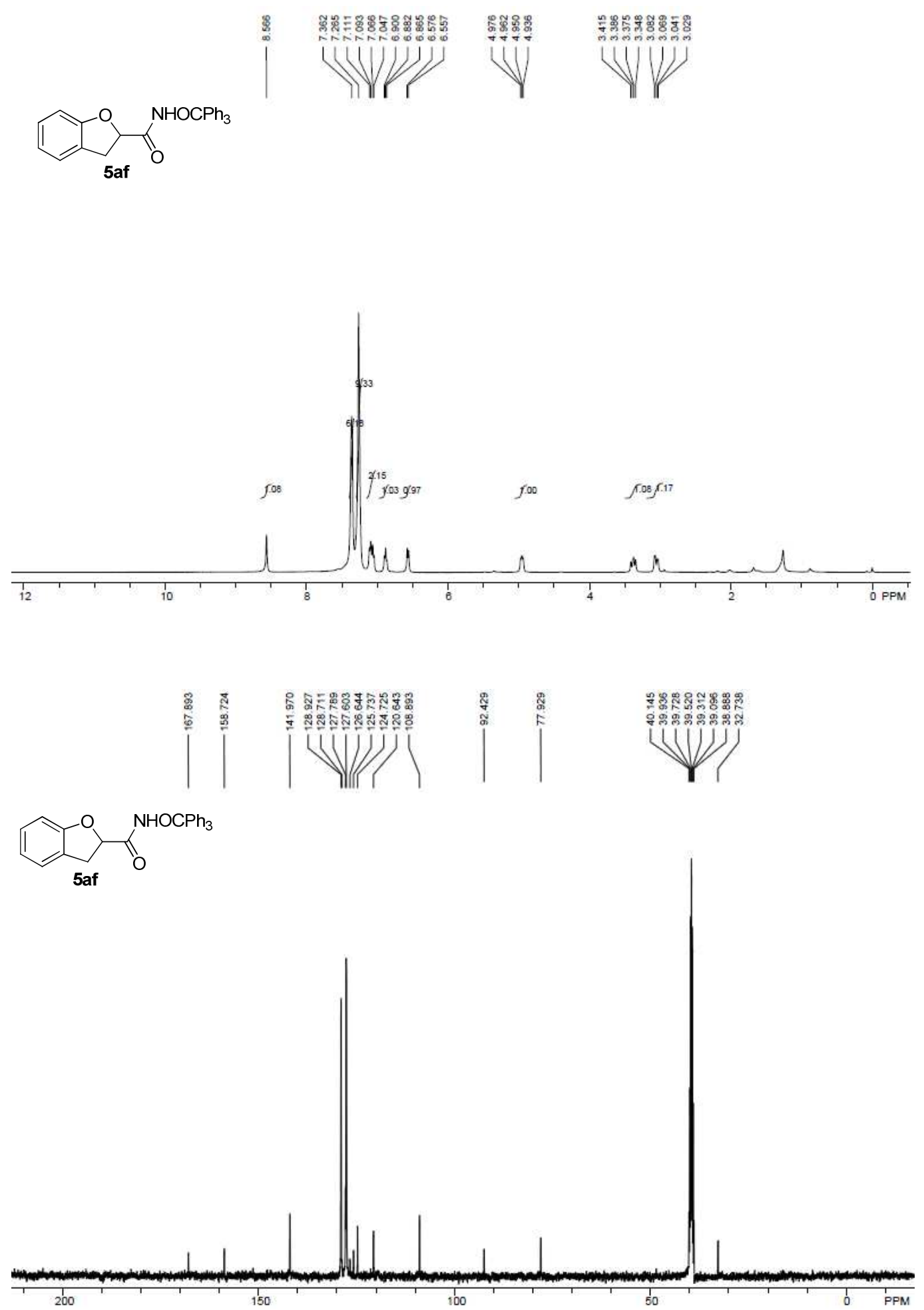